\title{
Electronic Properties of Graphene \\ Heterostructures with Hexagonal Crystals
}

John Robert Wallbank

PhD Thesis

Submitted for the degree of Doctor of Philosophy

November 26, 2013

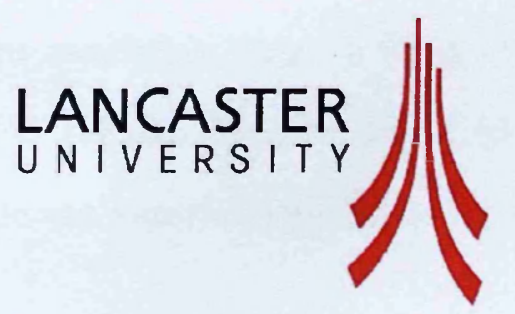


ProQuest Number: 11003766

All rights reserved

INFORMATION TO ALL USERS

The quality of this reproduction is dependent upon the quality of the copy submitted.

In the unlikely event that the author did not send a complete manuscript and there are missing pages, these will be noted. Also, if material had to be removed, a note will indicate the deletion.

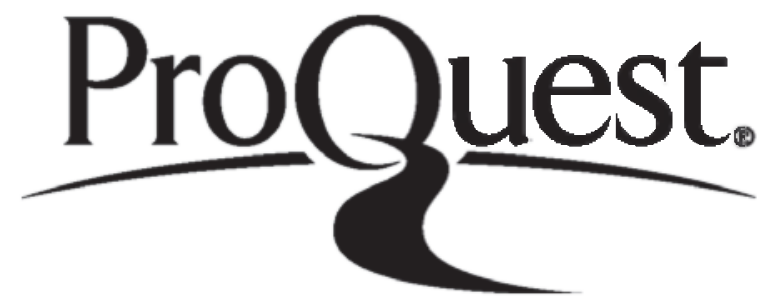

ProQuest 11003766

Published by ProQuest LLC (2018). Copyright of the Dissertation is held by the Author.

All rights reserved.

This work is protected against unauthorized copying under Title 17, United States Code Microform Edition (C) ProQuest LLC.

ProQuest LLC.

789 East Eisenhower Parkway

P.O. Box 1346

Ann Arbor, Ml $48106-1346$ 


\section{Declaration}

Except where stated otherwise, this Thesis is a result of the author's original work and has not been submitted in whole or in part for the award of a higher degree elsewhere. The following parts of the Thesis have been published, or submitted for publication, in peer reviewed journals:

Section 1.11. Unpublished original result obtained in collaboration with V. Cheianov and V. I. Falko.

Parts of chapter 2 published in J. R. Wallbank, A. A. Patel, M. MuchaKruczyński, A. K. Geim and V. I. Falko. Generic miniband structure of graphene on a hexagonal substrate. Phys. Rev. B 87, 245408 (2013).

Parts of chapter 3 preprint available from D. S. L. Abergel, J. R. Wallbank, Xi Chen, M. Mucha-Kruczyski, V. I. Fal'ko. Infrared absorption by graphene-hBN heterostructures. arXiv:1309.2292

Parts of chapter 4 preprint available from Xi Chen, J. R. Wallbank, M. MuchaKruczyński, E. McCann and V. I. Falko. Dirac edges of fractal magnetic minibands in graphene with hexagonal moire superlattices. arXiv:1310.8578

Parts of chapter 6 published in M. Mucha-Kruczyński, J. R. Wallbank, and V. I. Falko. Bilayer graphene heterostructure with hBN: Interplay between misalignment, interlayer asymmetry, and trigonal warping. Phys. Rev. B 88, 205418 (2013)

Parts of chapter 7 published in J. R. Wallbank, M. Mucha-Kruczyński, and V. I. Falko. Moire minibands in graphene heterostructures with almost commen- 
surate $\sqrt{3} \times \sqrt{3}$ hexagonal crystals. Phys. Rev. B 88, 155415 (2013).

Parts of chapter 8 published in R. Ferone, J. R. Wallbank, V. Zólyomi, E. McCann, V. I. Falko. Manifestation of LO-LA phonons in Raman scattering in graphene. Solid State Commun., 1511071 (2011)

Parts of chapter 8 Unpublished original result obtained in collaboration with V. I. Fal'ko.

John Robert Wallbank

November 26, 2013 


\section{Abstract}

In this Thesis, I provide a theoretical description of the properties of graphene on atomically flat hexagonal substrates, such as hexagonal boron nitride (hBN). It is known that the electronic properties of graphene-based devices can be dramatically improved by the use of such substrates. At the same time, a small lattice mismatch or misalignment angle, results in the formation of the large quasi-periodic structure known as a moiré pattern. The dominant effect of this, on graphene's electrons, can be described in terms of scattering by the simplest harmonics of the moiré pattern, which, combined with the symmetry of the system, allows a generic phenomenological Hamiltonian to be written. We systematically investigate the characteristic features that appear in the resulting miniband spectrum, and show that there generally exists additional secondary Dirac points, isolated on the energy axis, on the edge of the first moire miniband. This analysis is extended to bilayer-graphene/hBN heterostructures, which generically feature a gap at the edge of the first moiré miniband.

In a strong magnetic field, we find that generations of gapped Dirac electrons systematically reappear in Zak's magnetic miniband spectra, for rational values of the magnetic flux through the moire supercell. The fractal Hofstadter spectra, in the vicinity of such flux, can be described in terms of Landau levels, traced to the recurrent gapped Dirac electrons. Since this Landau level spectrum contains a zeroth energy level, separated by the largest gap from the rest of the spectrum, this determines a specific hierarchy of minigaps in the Hofstadter butterfly, and a peculiar sequence of dominant incompressible electron states.

By studying semiconductor materials databases, one finds that there are also several crystals with hexagonal facets almost commensurate with the $\sqrt{3} \times \sqrt{3}$ Kekulé lattice of graphene: InAs(111)B, InP(111)B, PdTe2, PtTe2, InSe, hGaTe. Using generic phenomenological theory, for superlattice effects created by such substrates on the Dirac electrons in graphene, we find that a typical miniband 
spectrum has a band gap between the first two minibands.

Separately, a theory of the electron-phonon coupling and Raman scattering by phonons in graphene is developed. Also, we systematically investigate the effect of moiré superlattice perturbation on graphene's, otherwise featureless, optical absorption spectra. 


\section{Acknowledgements}

The first acknowledgement in any Thesis should, in general, be reserved for one's supervisor. This Thesis is no exception. The tireless hard work and consistently good ideas of Professor Fal'ko have been the driving force behind all the material presented in this Thesis.

Moreover, I would like to thank all the people I have had the pleasure of working with during the course of my $\mathrm{PhD}$. A special thanks goes to both A. A. Patel, the first person at Lancaster to calculate the Hofstadter spectra, and M. MuchaKruczyński, who has worked with me on most of the topics presented here, and has been an excellent role model. Also, thanks to D. Abergel, who contributed to the material on optical absorption, E. McCann, R. Ferrone and V. Zóloymi for their help with the electron-phonon coupling, as well as Chen Xi and D. Gradinar.

Finally I would like to thank my family, including both Wallbanks and Garnseys, for their support, and in particular, Frances Garnsey and my grandma. 


\section{Contents}

1 Introduction $\quad 12$

1.1 Introduction . . . . . . . . . . . . . . . . . . 12

1.2 Geometry of Monolayer Graphene . . . . . . . . . . . . . . 14

1.3 Tight Binding Model for Monolayer Graphene . . . . . . . . . . . 14

$1.4 \boldsymbol{k} \cdot \boldsymbol{p}$ Model for Graphene . . . . . . . . . . . . . . . . . . . . 17

1.5 Symmetries of Graphene . . . . . . . . . . . . . . . . . 19

1.5.1 Geometrical Symmetries . . . . . . . . . . . . . . . . . 19

1.5.2 Matrix Representation of $c_{6 v} v^{\prime \prime} \ldots \ldots \ldots \ldots$

1.5.3 Symmetries and Degeneracies . . . . . . . . . . . . 21

1.5.4 Time Reversal Symmetry . . . . . . . . . . . . . . . . 21

1.6 Coupling to the Electromagnetic Field . . . . . . . . . . . 22

1.7 Landau Levels Spectra . . . . . . . . . . . . . . . . . . 23

1.8 Spectra of Bilayer Graphene . . . . . . . . . . . . 25

1.9 Hexagonal Boron Nitride . . . . . . . . . . . . . . . 26

1.10 The Moiré Pattern . . . . . . . . . . . . . . 27

1.11 The Moiré Magnifying Glass . . . . . . . . . . . . . . . 30

2 Monolayer Graphene on a hBN underlay 33

2.1 Introduction . . . . . . . . . . . . . 33

2.2 Superlattice Hamiltonian $\ldots \ldots \ldots \ldots$

2.3 Generic Miniband Spectra of Graphene-hBN Heterostructures . . . 37

2.4 Effect of the Miniband Spectra on the Hall Coefficent . . . . . . . 41 
2.5 Symmetry of the Moiré Superlattice . . . . . . . . . . . . 43

2.6 Microscopic Models . . . . . . . . . . . . . . . . . . . . . 45

2.6.1 Point Charge Model . . . . . . . . . . . . . . . . 45

2.6.2 Graphene-hBN Hopping Model . . . . . . . . . . . . . 47

2.6.3 Inversion Asymmetric Microscopic Models . . . . . . . . . 48

2.7 Conclusion . . . . . . . . . . . . . . . . . . . . . 49

3 Optical Absorption in Graphene-hBN Heterostructures $\quad 51$

3.1 Introduction . . . . . . . . . . . . . . . . . 51

3.2 Optical Absorption Spectra $\ldots \ldots \ldots \ldots \ldots$

3.3 Conclusion . . . . . . . . . . . . . . . . 56

4 Fractal Spectrum of Magnetic Minibands in Graphene-hBN Heterostructures

4.1 Introduction . . . . . . . . . . . . . . . . 59

4.2 Magnetic Translational Symmetry and Calculation of Zak's Magnetic Minibands . . . . . . . . . . . . . . . . . . 61

4.3 Generic Features in the Magnetic Miniband Spectra . . . . . . . 65

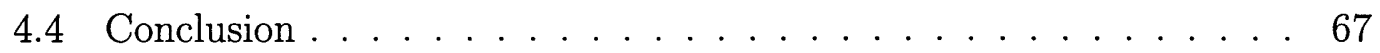

5 Experimental Realisation of the Graphene-hBN Heterostructure 69

6 Bilayer Graphene on hBN $\quad 72$

6.1 Introduction . . . . . . . . . . . . . 72

6.2 Moiré Superlattice Hamiltonian . . . . . . . . . . . . . . 73

6.3 Symmetries in the Miniband Structure . . . . . . . . 76

6.4 Low-energy Hamiltonian for BLG-hBN Heterostructures . . . . 77

6.5 Miniband Spectra of BLG-hBN Heterostructures $\ldots \ldots \ldots . .78$

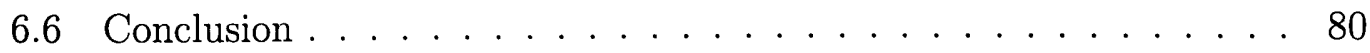

7 Monolayer Graphene with Almost Commensurate $\sqrt{3} \times \sqrt{3}$ Hexagonal Crystals 
7.1 Introduction . . . . . . . . . . . . . . . . . . 84

7.2 Phenomenological Superlattice Hamiltonian $\ldots \ldots \ldots$

7.3 Microscopic Models . . . . . . . . . . . . . . . . . 87

7.4 Parameter Space of the Superlattice Perturbation . . . . . . . 88

7.5 Translational Symmetries and Zone Folding . . . . . . . . . . . 89

7.6 Characteristic Miniband Spectra . . . . . . . . . . . . 90

7.7 Conclusion . . . . . . . . . . . . . . . . . . . . . . . . 92

8 Raman Scattering by Phonons in Graphene $\quad 95$

8.1 Introduction . . . . . . . . . . . . . . . 95

8.2 In-plane Phonon Modes $\ldots \ldots$. . . . . . . . . . . . 97

8.3 Electron-Phonon Coupling . . . . . . . . . . . . . . . . . . 99

8.4 LO-LA Raman Spectra . . . . . . . . . . . . . . . . . . . . . 102

8.5 Renormalisation of the $\mathrm{TO}_{K_{ \pm}}$Phonon for Graphene on hBN . . . 105

8.5.1 Vertex Correction Due to the Superlattice Perturbation . . . 106

8.5.2 $\mathrm{TO}_{K_{ \pm}}$Self-Energy Calculation . . . . . . . . . 106

8.6 Conclusion . . . . . . . . . . . . . . . . . . . . . . 109

9 Conclusion

Appendix

A Minibands in the Vicinity of $\kappa$ and $\mu$-points of Graphene-hBN Heterostructures

A.1 $\mu$-point Hamiltonian . . . . . . . . . . . . . . . . 112

A.2 Hamiltonian at the $\kappa$-point . . . . . . . . . . 113

B Minibands of Graphene with Almost Commensurate $\sqrt{3} \times \sqrt{3}$ Hexagonal Crystals 


\section{List of Figures}

1.1 The graphene lattice . . . . . . . . . . . . . . . . . . 14

1.2 The tight binding bandstructure of graphene . . . . . . . 17

1.3 Transformation of the Bloch wavefunctions under $c_{6 v^{\prime \prime}} \ldots \ldots 23$

1.4 The Landau levels of the Dirac Hamiltonian . . . . . . . . . . 24

1.5 The crystal structure and bandstructure of $A-B$ stacked bilayer

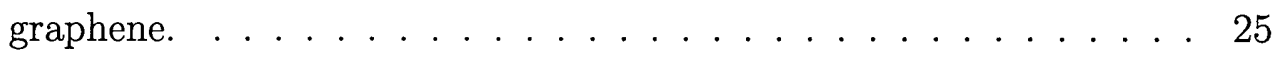

1.6 The magnifying effect of the moiré pattern . . . . . . . . . 29

2.1 The moiré Brillouin zone and phase diagram . . . . . . . 34

2.2 Numerically calculated moiré miniband . . . . . . . . . . . 38

2.3 The two densities at which the Hall coefficient in graphene reverses

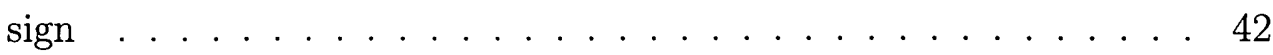

2.4 Point charge model . . . . . . . . . . . . . . . . 47

2.5 Miniband spectra corresponding to the microscopic model . . . . . 48

3.1 Absorption spectra for the model moiré perturbations . . . . . . . . 54

3.2 Absorption spectra for each of the symmetry-allowed superlattice perturbation ..................... 57

3.3 Variation of the optical spectrum with Fermi energy . . . . . . 58

4.1 Moiré pattern of graphene on a hBN underlay . . . . . . . . 61

4.2 Magnetic Brillouin zones . . . . . . . . . . . . . . . . 62

4.3 Magnetic miniband spectrum $\ldots \ldots \ldots \ldots \ldots$ 
4.4 Fan diagram for graphene-hBN heterostructures in a strong magnetic field. . . . . . . . . . . . . . . . . . 68

5.1 Experimentally measured longitudinal and Hall resistivities . . . . 70

5.2 Experimentally measured signature of the Hofstadter spectra . . . . 71

6.1 Parameter space $\left(u_{0}, u_{1}, u_{3}\right)$ of the in BLG-hBN superlattice per-

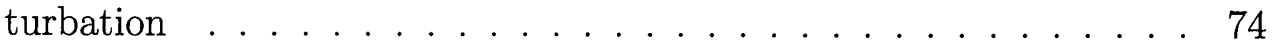

6.2 The Brillouin zone and BLG mini Brillouin zone of BLG . . . . 75

6.3 Moiré miniband spectra . . . . . . . . . . . . . . . . . 82

6.4 Size of the gap between the first and second minibands . . . . . . 83

6.5 The role of trigonal warping in BLB-hBN heterostructures. . . . . . 83

7.1 Moiré pattern on a $\sqrt{3} \times \sqrt{3}$ substrate $\ldots \ldots \ldots \ldots$

7.2 Numerically calculated moiré minibands . . . . . . . . . . . . 91

7.3 Parameter space of the superlattice perturbation . . . . . . . . 92

8.1 The in-plane phonon modes and Raman spectra of graphene . . . . 96

8.2 Resonant two-phonon scattering in graphene . . . . . . . . . . . 102

8.3 Lineshapes of the LA mode and LO mode . . . . . . . . . . . 105

8.4 Contributions to the self energy of the $\mathrm{TO}_{K_{ \pm}}$phonon for the graphene/hBN heterostructure . . . . . . . . . . . . . . 107

8.5 The $\mathrm{TO}_{K_{+}}$phonon energy $\omega_{T O}\left(q_{0}, \theta_{q}\right)$ and Raman spectra for the graphene $/$ hBN heterostructure . . . . . . . . . . . . . . . 109

B.1 Further examples of numerically calculated moiré minibands for graphene on $\sqrt{3} \times \sqrt{3}$ hexagonal crystals. . . . . . . . . . 116 


\section{List of Tables}

1.1 Character table for $c_{6 v^{\prime \prime}} \ldots \ldots \ldots \ldots \ldots \ldots$

2.1 Microscopic models for the graphene-hBN heterostructure $\ldots \ldots 36$

7.1 Surfaces almost commensurate with the $\sqrt{3} \times \sqrt{3}$ graphene super-

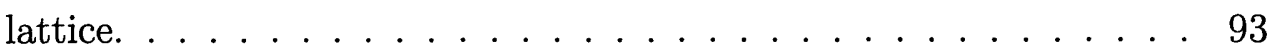

8.1 DFT values of parameters $\epsilon^{\prime}$ and $t^{\prime} \ldots \ldots \ldots \ldots 10 \ldots \ldots$ 


\section{Chapter 1}

\section{Introduction}

\section{$1.1 \quad$ Introduction}

The theory of graphene has its roots in the tight binding models of graphite [13], later used to model the spectra of fullerene molecules [4] and nanotubes [5]. However, it was only more recently that, in 2004, the group of Andre Geim first succeeded in using their mechanical exfoliation technique to isolate a single layer of graphite: graphene [6]. Not only was this the first two-dimensional carbon allotrope, it was the first ever truly two-dimensional material. And it would not be the last. Barely a year later the same exfoliation technique was applied to a variety of layered materials: boron nitride, dichalcogenides and complex oxides [7]. Since then, there has been rapid progress in the field of graphene, and many of the "low-hanging research fruits" have already been plucked. Because of this it is now the other two-dimensional materials, and their heterostructures, that look set to receive increased attention [8].

There is one particular graphene heterostructure that is almost always present in any graphene device; that is the heterostructure formed between graphene and its substrate. At first, with the use of the $\mathrm{SiO}_{2}$ substrate, this was regarded as a mere nuisance. The graphene electrons experience scattering from impurities in the substrate, leading to a reduced mobility, and electron-hole puddles that hide the graphene Dirac point from the experimentalist [9-11]. One approach to 
prevent this is the clever use of suspended graphene samples $[16,17]$.

A simpler approach to obtain high quality devices is through the correct choice of substrate, with atomically flat hexagonal boron-nitride (hBN) proving particularly good in this role [9-15]. However, the substrate can play more than a merely mechanical role in the structure of the device. Rather, the substrate can qualitatively modify the graphene spectra, providing new opportunities to tailor the graphene bandstructure and observe new physical phenomena. With regard to the well aligned graphene on hBN system, the small lattice mismatch between the graphene and $\mathrm{hBN}$ lattices generates the long-period interference pattern known as the moiré superlattice. The superlattice potential [18-21] thus created results in the formation of replica secondary Dirac points in the graphene spectra, which have recently been observed experimentally [22-25]. Moreover, the well aligned graphene-hBN system is not just of academic interest, obtainable only through the careful alignment of the graphene and $\mathrm{hBN}$ crystallographic directions. Instead, this system arises naturally during the epitaxial growth [26] of graphene on the hBN surface. Finally, there are many hexagonal surfaces with the unit cell area almost three times that of graphene. We will later show that these give rise to their own unique type of superlattice perturbation, which allows for the opening of band gaps in graphene's spectrum, whilst, simultaneously, offering the prospect of good mobility.

This Thesis is organised as follows: In the first chapter, we review the key theoretical background used in the rest of the Thesis. The next four chapters will be devoted to the graphene-hBN heterostructure: Chapter 2 describing the phenomenological model for this system, and chapter 3 discussing the optical absorption and electronic transport properties. Chapter 4 is devoted to the peculiar fractal properties of the Hofstadter spectrum of the graphene-hBN heterostructure in a strong magnetic field. The manifestation of these features in recent experimental results is discussed in chapter 5 . In chapter 6 , we move on to the bilayer graphene-hBN heterostructure. In chapter 7 , we consider alternative hexagonal 
substrates, almost commensurate with the $\sqrt{3} \times \sqrt{3}$ Kekulé lattice of graphene. Finally, in chapter 8, we review the electron-phonon coupling, and Raman spectra in graphene and graphene-hBN heterostructures. Some brief concluding remarks are made in chapter 9 .

\subsection{Geometry of Monolayer Graphene}

a)

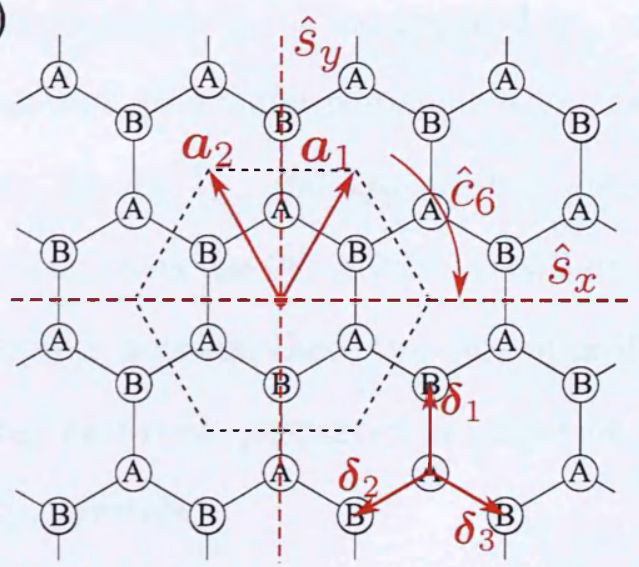

b)

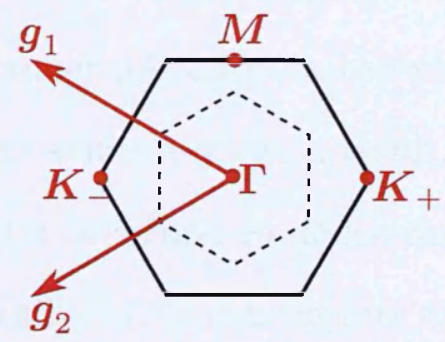

Figure 1.1: a) The real space graphene lattice. Showing the two carbon atoms per unit cell $(A$ and $B)$, the primitive lattice vectors, and the point group symmetry operations (see section 1.5). b) The graphene Brillouin zone, with symmetry points and reciprocal lattice vectors labelled. The dashed lines in (a) and (b) show the tripled graphene unit cell and its Brillouin zone.

Graphene has the honeycomb lattice (Fig. 1.1 (a)), with two carbon sites per unit cell, known as the $A$ and $B$ sites. The primitive lattice vectors are $\boldsymbol{a}_{1}=$ $a(1 / 2, \sqrt{3} / 2)$, and $\boldsymbol{a}_{2}=a(-1 / 2, \sqrt{3} / 2)$, where $a=2.46 \AA$ is the graphene lattice constant $[27,28]$. The nearest neighbour vectors are $\boldsymbol{\delta}_{j}=\hat{R}_{2 \pi j / 3}(0, a / \sqrt{3})$, with $\hat{R}_{2 \pi j / 3}$ the anticlockwise rotation matrix.

In reciprocal space (Fig. $1.1(\mathrm{~b})) \boldsymbol{g}_{1}=\frac{4 \pi}{\sqrt{3} a}\left(\frac{-\sqrt{3}}{2}, \frac{1}{2}\right)$ and $\boldsymbol{g}_{2}=\frac{4 \pi}{\sqrt{3} a}\left(\frac{-\sqrt{3}}{2},-\frac{1}{2}\right)$; the $\boldsymbol{K}_{ \pm}=\frac{4 \pi}{3 a}( \pm 1,0)$ are referred to as graphene's two valleys; and $\boldsymbol{M}=\left(0, \frac{4 \pi}{2 \sqrt{3} a}\right)$.

\subsection{Tight Binding Model for Monolayer Graphene}

The majority of the material found in this Thesis relies on a minimal tight binding model of graphene, which we now briefly summarise. More detailed descriptions 
are found in Refs. [27, 29, 30].

The tight binding model is based on the diagonalisation of the microscopic Hamiltonian in a basis of the atomic orbitals on the carbon atoms (or some generalisation thereof). Each carbon atom provides six electrons, two of which belong to the core, with the remainder occupying the $2 s, 2 p_{x}, 2 p_{y}$, and $2 p_{z}$ orbitals. Of these, the low-energy electronic properties of graphene are controlled by the carbon $2 p_{z}$ orbitals, which are directed perpendicularly to the graphene plane. The remaining electrons in the $2 s, 2 p_{x}$ and $2 p_{y}$ orbitals, are hybridised into a $s p^{2}$ configuration and are chiefly responsible for the $120^{\circ}$ bond angles of graphene [27]. Moreover, the $2 s, 2 p_{x}$ and $2 p_{y}$ orbitals are even under reflection in the plane of graphene, whereas the $2 p_{z}$ orbital is odd under this symmetry. As a result, there is no coupling between these two sets of orbitals and therefore, to understand the low-energy electronic properties of graphene, it is sufficient to concentrate entirely on the $2 p_{z}$ orbitals.

Due to the translational symmetry of the graphene lattice, any wavefunction, $\psi_{k}$, may be characterised by a wavevector $\boldsymbol{k}$ [31]. The wavevector is defined, within the Brillouin zone shown in Fig. 1.1 (b), by,

$$
\hat{T}_{\boldsymbol{R}} \psi_{k}(\boldsymbol{r})=e^{i \boldsymbol{k} \cdot \boldsymbol{R}} \psi_{k}(\boldsymbol{r})
$$

where $\hat{T}_{\boldsymbol{R}}$ is the translational operator, $\hat{T}_{\boldsymbol{R}} f(\boldsymbol{r})=f(\boldsymbol{r}+\boldsymbol{R})$. Because of this we must form linear combinations of the $2 p_{z}$ orbitals which satisfy Eq. (1.1). To do this we consider the Bloch wavefunctions,

$$
\Phi_{i, k}(\boldsymbol{r})=\frac{1}{\sqrt{N}} \sum_{\boldsymbol{R}} e^{i \boldsymbol{k} \cdot\left(\boldsymbol{R}+\boldsymbol{\tau}_{i}\right)} \phi_{\mathrm{pz}}\left(\boldsymbol{r}-\boldsymbol{R}-\boldsymbol{\tau}_{i}\right)
$$

where $i=A, B$ labels the two carbon sites in the unit cell, and $\phi_{\mathrm{pz}}(\boldsymbol{r})$ is the normalised carbon $2 p_{z}$ orbital. The sum runs over all hexagon centre sites in the crystal, $N$ is the number of unit cells in the crystal, and $\boldsymbol{\tau}_{A / B}= \pm \boldsymbol{\delta}_{1}$. To make rapid progress I use the simplifying assumptions that: 
(i) The $\phi_{\mathrm{pz}}(\boldsymbol{r})$ wavefunctions decay so rapidly at $\boldsymbol{r} \lesssim a / \sqrt{3}$ that the Bloch functions on the $A$ and $B$ sites are orthogonal.

(ii) Hopping is limited to nearest neighbours only,

$$
\left\langle\phi_{\mathrm{pz}}\left(\boldsymbol{r}-\boldsymbol{R}^{\prime}-\boldsymbol{\tau}_{B}\right)|H| \phi_{\mathrm{pz}}\left(\boldsymbol{r}-\boldsymbol{R}-\boldsymbol{\tau}_{A}\right)\right\rangle= \begin{cases}-\gamma_{0} & \text { for }\left(\boldsymbol{R}^{\prime}+\boldsymbol{\tau}_{B}\right)-\left(\boldsymbol{R}+\boldsymbol{\tau}_{A}\right)=\boldsymbol{\delta}_{j=1,2,3} \\ 0 & \text { otherwise }\end{cases}
$$

where $\gamma_{0} \approx 3.0 \mathrm{eV}[27]$ is positive. Additionally, I set the on-site energy of the $2 p_{z}$ orbital in the graphene lattice equal to zero, that is $\left\langle\phi_{\mathrm{pz}}|H| \phi_{\mathrm{pz}}\right\rangle \equiv 0$

With these approximations it is immediately possible to write down the Hamiltonian in the basis of Bloch wavefunctions $\left(\Phi_{A, \boldsymbol{k}}, \Phi_{B, \boldsymbol{k}}\right)$,

$$
\hat{H}=-\gamma_{0}\left(\begin{array}{cc}
0 & f(\boldsymbol{k}) \\
f^{*}(\boldsymbol{k}) & 0
\end{array}\right), \quad f(\boldsymbol{k}) \equiv \sum_{j=1,2,3} e^{i \boldsymbol{k} \cdot \boldsymbol{\delta}_{j}}
$$

The Hamiltonian is readily diagonalisable to give two bands,

$$
\epsilon_{s}(\boldsymbol{k})=s|f(\boldsymbol{k})|, \quad s= \pm 1,
$$

which are plotted in Fig. 1.2. The electron-hole symmetry present in Eq. (1.4) is merely an artefact of the approximations used, and is lifted if the non-orthogonality of the Bloch functions is accounted for $[27,30]$.

For undoped graphene, one electron from each of graphene's two carbon sites occupies the $\pi$-bands. Hence, counting spin degeneracy, the Fermi level sits at $\epsilon=0$, so that the lower branch $(s=-1)$ is completely filled, while the higher branch $(s=1)$ is completely empty. The two branches meet at the Brillouin zone corners with a linear dispersion which we discuss in the next section. 


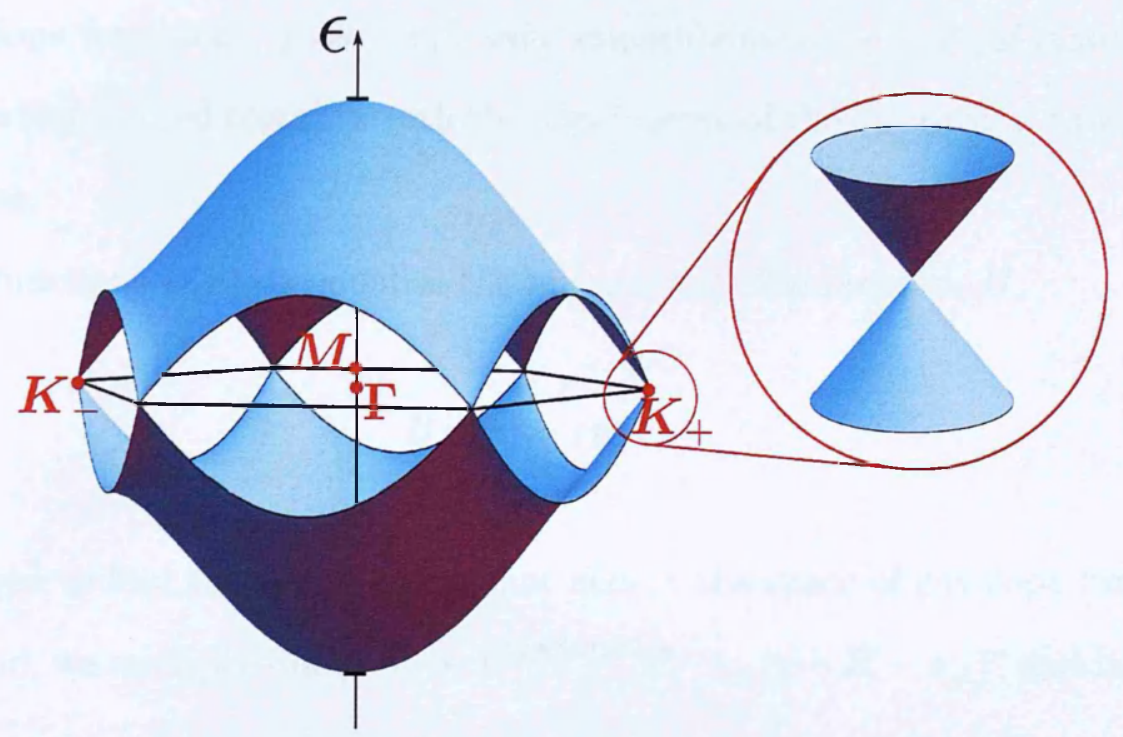

Figure 1.2: The bandstructure of graphene, in the first Brillouin zone, calculated from Eq. (1.4). The bandwidth is set by energies $\pm 3 \gamma_{0}$ which occur at the $\Gamma$-point, while energies $\pm \gamma_{0}$ correspond to a Van-Hove singularity, due to the saddle-point occurring at the $M$-point. The inset shows the linear dispersion found at the Brillouin zone corners.

\section{$1.4 k \cdot p$ Model for Graphene}

To understand the electronic properties of any material, particular attention must be paid to the electronic states around the Fermi level. To this end, we use $\boldsymbol{k} \cdot \boldsymbol{p}$ theory [32-34] to derive the the Dirac Hamiltonian, describing the low energy dispersion around graphene's Brillouin zone corners. To do this we assume that the low-energy wavefunction, taking states from the $K_{+}$valley only*, can be written in the form,

$$
\begin{aligned}
\psi(\boldsymbol{r}) & =\frac{1}{\sqrt{N}} \sum_{i=A / B} \sum_{\boldsymbol{R}} f_{i}\left(\boldsymbol{R}+\boldsymbol{\tau}_{i}\right) e^{i \boldsymbol{K}_{+} \cdot\left(\boldsymbol{R}+\boldsymbol{\tau}_{i}\right)} \phi_{p z}\left(\boldsymbol{r}-\boldsymbol{R}-\boldsymbol{\tau}_{i}\right) \\
& \approx \sum_{i=A / B} f_{i}(\boldsymbol{r}) \frac{1}{\sqrt{N}} \sum_{\boldsymbol{R}} e^{i \boldsymbol{K}_{+} \cdot\left(\boldsymbol{R}+\boldsymbol{\tau}_{i}\right)} \phi_{p z}\left(\boldsymbol{r}-\boldsymbol{R}-\boldsymbol{\tau}_{i}\right) .
\end{aligned}
$$

*In reality, states from both valleys make up the low-energy wavefunctions (see treatment in, e.g., Ref. [34]). However, states with different wavevectors do not couple so the treatment here is sufficient. 
The envelope functions ${ }^{\dagger}, f_{i}\left(\boldsymbol{R}+\boldsymbol{\tau}_{i}\right)$, vary smoothly over the scale of many lattice periods, which is used together with the rapid decay of the $2 p_{z}$ orbital to write the second line.

Wavefunctions $\psi(\boldsymbol{r})$ diagonalise the microscopic Hamiltonian, $H$,

$$
H \psi(\boldsymbol{r})=\epsilon \psi(\boldsymbol{r})
$$

This is used to find the Hamiltonian that acts in the space of envelope functions. To this end, we multiply Eq. (1.6) by $e^{-i \boldsymbol{K}_{+} \cdot\left(\boldsymbol{R}^{\prime}+\boldsymbol{\tau}_{A}\right)} \phi_{p z}\left(\boldsymbol{r}-\boldsymbol{R}^{\prime}-\boldsymbol{\tau}_{A}\right)^{*}$ and integrate to get,

$$
\epsilon f_{A}\left(\boldsymbol{R}^{\prime}+\boldsymbol{\tau}_{A}\right)=-\gamma_{0} \sum_{\delta_{i=1,2,3}} f_{B}\left(\boldsymbol{R}^{\prime}+\boldsymbol{\tau}_{A}+\boldsymbol{\delta}_{i}\right) e^{i \boldsymbol{K}_{+} \cdot \boldsymbol{\delta}_{i}}
$$

where we have used the same assumptions and notations as section 1.3. We now Taylor expand $f_{B}\left(\boldsymbol{R}^{\prime}+\boldsymbol{\tau}_{A}+\boldsymbol{\delta}_{i}\right)$ in $\boldsymbol{\delta}_{i}$ to get,

$$
\begin{aligned}
& \epsilon f_{A}(r) \approx-\gamma_{0} \sum_{\boldsymbol{\delta}_{i=1,2,3}}\left[f_{B}(r)+\left(\boldsymbol{\delta}_{i} \cdot \nabla\right) f_{A}(r)+\cdots\right] e^{i \boldsymbol{K}_{+} \cdot \boldsymbol{\delta}_{i}} \\
& =\frac{\sqrt{3}}{2} a \gamma_{0}\left[-\partial_{y}-i \partial_{x}\right] f_{B}(r) .
\end{aligned}
$$

Writing the similar expression for $f_{B}$ gives [35],

$$
v\left(\begin{array}{cc}
0 & \pi^{\dagger} \\
\pi & 0
\end{array}\right) \boldsymbol{\psi}(\boldsymbol{r})=\epsilon \boldsymbol{\psi}(\boldsymbol{r}), \quad \boldsymbol{\psi}(\boldsymbol{r})=\left(\begin{array}{c}
f_{A}(r) \\
f_{B}(r)
\end{array}\right), \quad \hat{\pi}=\hat{p}_{x}+i \hat{p}_{y}
$$

Here, we use the basis $\left(\Phi_{A, K_{+}}, \Phi_{B, K_{+}}\right)$, momentum operators $\hat{p}_{x_{i}}=-i \partial_{x_{i}}$, and the Dirac velocity $v=\frac{\sqrt{3}}{2} a \gamma_{0}$. The matrix, acting on the left of $\boldsymbol{\psi}$ in Eq. (1.10), is identified as the Dirac Hamiltonian, and can be written as $v \boldsymbol{p} \cdot \boldsymbol{\sigma}$, where $\boldsymbol{\sigma}=\left(\sigma_{1}, \sigma_{2}\right)$ is the vector of Pauli matrices. The Hamiltonian in the vicinity of the opposite valley is the same as Hamiltonian (1.10) and has the basis $\left(\Phi_{B, K_{-}},-\Phi_{A, K_{-}}\right)$.

${ }^{\dagger}$ Taking $f_{i}\left(\boldsymbol{R}+\tau_{i}\right)=c_{i} e^{i \boldsymbol{k} \cdot\left(\boldsymbol{R}+\tau_{i}\right)}$ in the top line of Eq. (1.5) is equivalent to using Bloch states (1.2). 
Diagonalisation gives

$$
\epsilon_{s}(\boldsymbol{k})=s v|\boldsymbol{k}|, \quad \boldsymbol{\psi}_{s= \pm, k}(\boldsymbol{r})=\frac{1}{\sqrt{2} L}\left(\begin{array}{c}
1 \\
s e^{i \theta_{\boldsymbol{k}}}
\end{array}\right) e^{i \boldsymbol{k} \cdot \boldsymbol{r}}
$$

where $\boldsymbol{k}$ is measured with respect to the valley centre. The same dispersion relation is obtained by expanding tight binding Hamiltonian (1.3) at momentum $\boldsymbol{K}_{+}+\boldsymbol{k}$ for $\boldsymbol{k} \ll 1 / a$.

\subsection{Symmetries of Graphene}

\subsubsection{Geometrical Symmetries}

Table 1.1: The character table for $c_{6 v^{\prime \prime}}$. The right hand column shows the basis of matrices, acting on the space of graphene's four-component electronic states, which transform according to each irreducible representation.

\begin{tabular}{|c|c|c|c|c|c|c|c|c|c|c|}
\hline$c_{6 v^{\prime \prime}}:$ & 1 & $\begin{array}{l}T_{a_{1}} \\
T_{a_{2}}\end{array}$ & $\begin{array}{c}c_{6}^{3} \\
T_{\boldsymbol{a}_{2}} c_{6}^{3} \\
T_{\boldsymbol{a}_{1}} c_{6}^{3}\end{array}$ & $\begin{array}{l}c_{6}^{2} \\
c_{6}^{4}\end{array}$ & $\begin{array}{l}c_{6}^{2} T_{\boldsymbol{a}_{1}} \\
c_{6}^{4} T_{\boldsymbol{a}_{1}} \\
c_{6}^{2} T_{\boldsymbol{a}_{2}} \\
c_{6}^{4} T_{\boldsymbol{a}_{2}} \\
\end{array}$ & $\begin{array}{c}c_{6} \\
c_{6}^{5} \\
c_{6} T_{a_{1}} \\
c_{6}^{5} T_{a_{1}} \\
c_{6} T_{a_{2}} \\
c_{6}^{5} T_{a_{2}} \\
\end{array}$ & $\begin{array}{c}3 s_{y} \\
3 T_{a_{1}} s_{y} \\
3 T_{a_{2}} s_{y}\end{array}$ & $3 s_{x}$ & $\begin{array}{l}3 T_{a_{1}} s_{x} \\
3 T_{a_{2}} s_{x}\end{array}$ & \\
\hline $\mathrm{A}_{1}$ & 1 & 1 & 1 & 1 & 1 & 1 & 1 & 1 & 1 & 1 \\
\hline $\mathrm{B}_{2}$ & 1 & 1 & -1 & 1 & 1 & -1 & 1 & -1 & -1 & $\sigma_{3} \tau_{3}$ \\
\hline $\mathrm{A}_{2}$ & 1 & 1 & 1 & 1 & 1 & 1 & -1 & -1 & -1 & $\sigma_{3}$ \\
\hline $\mathrm{B}_{1}$ & 1 & 1 & -1 & 1 & 1 & -1 & -1 & 1 & 1 & $\tau_{3}$ \\
\hline $\mathrm{E}_{1}$ & 2 & 2 & -2 & -1 & -1 & 1 & 0 & 0 & 0 & $\sigma_{1}, \sigma_{2}$ \\
\hline $\mathrm{E}_{2}$ & 2 & 2 & 2 & -1 & -1 & -1 & 0 & 0 & 0 & $-\sigma_{2} \tau_{3}, \sigma_{1} \tau_{3}$ \\
\hline $\mathrm{E}^{\prime}$ & 2 & -1 & 0 & 2 & -1 & 0 & 0 & 2 & -1 & $\sigma_{3} \tau_{1}, \sigma_{3} \tau_{2}$ \\
\hline E" & 2 & -1 & 0 & 2 & -1 & 0 & 0 & -2 & 1 & $\tau_{1}, \tau_{2}$ \\
\hline $\mathrm{G}$ & $\overline{4}$ & -2 & 0 & -2 & 1 & 0 & 0 & 0 & 0 & $\begin{array}{c}\sigma_{2} \tau_{1},-\sigma_{1} \tau_{1} \\
\sigma_{1} \tau_{2}, \sigma_{2} \tau_{2} \\
\end{array}$ \\
\hline
\end{tabular}

An understanding of the symmetry $[36,37]$ of the graphene lattice is essential to understand many of its physical properties. The graphene lattice has the $c_{6 v}$ point group symmetry, the elements of which are shown in Fig. 1.1, and contains the $2 \pi / 6$ rotation, $\hat{c}_{6}$, the sublattices preserving reflection, $\hat{s}_{y}$, and the sublattice exchanging reflections, $\hat{s}_{x}$. However, we will only be interested in the properties 
of graphene's electrons or phonons in the neighbourhoods of the two inequivalent Brillouin zone corners, $\boldsymbol{K}_{+}$and $\boldsymbol{K}_{-}$. It is therefore more convenient to consider the symmetry group of the tripled graphene unit cell, $c_{6 v^{\prime \prime}}=c_{6 v}+\left(T_{\boldsymbol{a}_{1}} c_{6 v}\right)+\left(T_{a_{2}} c_{6 v}\right)$ [39]. The tripled graphene unit cell has a correspondingly three times smaller Brillouin zone, shown as a black dashed hexagon in Fig. 1.1 (b). Importantly, wavevectors $\boldsymbol{K}_{ \pm}$are folded onto the $\boldsymbol{\Gamma}$-point of this smaller Brillouin zone, which avoids having to work with the group of their wavevectors. The character table of $c_{6 v^{\prime \prime}}$ is given in table 1.1

\subsubsection{Matrix Representation of $c_{6 v^{\prime \prime}}$}

In the the spirit of the $\boldsymbol{k} \cdot \boldsymbol{p}$ approximation, any low-energy graphene electronic Hamiltonian can be written using $4 \times 4$ Hermitian matrices, acting in a basis of Bloch wave functions, $\left(\Phi_{A K_{+}}, \Phi_{B K_{+}}, \Phi_{B K_{-}},-\Phi_{A K_{-}}\right)$, on the $A$ and $B$ sublattices at the exact Brillouin zone corners. These matrices are conveniently written using the Kronecker products of two commuting sets of Pauli matrices $\sigma_{i}, \tau_{j}$, which are said to act on graphene's spin and valley degrees of freedom separately. Throughout this Thesis, the Kronecker product will be implied so that $\sigma_{i} \tau_{j} \equiv \tau_{j} \otimes \sigma_{i}$, and identity matrices are not written.

Upon application of a symmetry operation $\hat{G}$, the Bloch wavefunctions will transform into each other according to some matrix $\Gamma(G)$,

$$
\hat{G} \Phi_{i}(\boldsymbol{r}) \equiv \Phi_{i}\left(G^{-1} \boldsymbol{r}\right)=\sum_{j} \Gamma_{j i}(G) \Phi_{j}(\boldsymbol{r})
$$

Here $G^{-1}$ is the coordinate transformation corresponding to $\hat{G}$. The symmetry transformation therefore acts on the electronic matrices as,

$$
\hat{G} \sigma_{i} \tau_{j}=\Gamma(G) \sigma_{i} \tau_{j} \Gamma^{\dagger}(G)
$$

It is sufficient to list matrices $\Gamma(G)$ for the generators of $c_{6 v^{\prime \prime}}$. The matrix for spatial inversion symmetry, $\hat{I}=\hat{c}_{6}^{3}$, and reflection symmetry $\hat{s}_{y}$, are also listed for 
convenience,

$$
\begin{gathered}
\Gamma_{c_{6}}=\left(i \frac{\sqrt{3}}{2}-\frac{1}{2} \sigma_{3}\right) \tau_{1}, \quad \Gamma_{s_{x}}=\sigma_{1} \tau_{3}, \quad \Gamma_{\boldsymbol{a}_{1}}=\left(-\frac{1}{2}-i \frac{\sqrt{3}}{2} \tau_{3}\right) ; \\
\Gamma_{s_{y}}=\sigma_{2} \tau_{2}, \quad \Gamma_{I}=\sigma_{3} \tau_{1} .
\end{gathered}
$$

Figure 1.3 shows a convenient method of calculating these matrices, based on the application of geometrical symmetries to the Bloch wavefunctions. The combinations of $\sigma_{i}$ and $\tau_{j}$, that transform according to the irreducible representations of $c_{6 v^{\prime \prime}}$, are shown in table 1.1.

\subsubsection{Symmetries and Degeneracies}

The Hamiltonian for a system must remain invariant under all symmetries the system possesses. Constructing invariant combinations of the $\sigma_{i} \tau_{j}$ matrices with the other dependencies in the Hamiltonian is a convenient alternative to microscopic calculation, which will be used in full in the following chapters. That is,

$$
\hat{G} H(\boldsymbol{r}) \equiv \Gamma(G) H\left(G^{-1} \boldsymbol{r}\right) \Gamma^{\dagger}(G)=H(\boldsymbol{r})
$$

must be obeyed if the system possesses symmetry $G$. Moreover, each symmetry of the Hamiltonian results in a degeneracy between wavefunctions $\psi$ and $\hat{G} \psi \equiv$ $\Gamma(G) \psi\left(G^{-1} \boldsymbol{r}\right)$.

\subsubsection{Time Reversal Symmetry}

The effect of time reversal symmetry, $\hat{T}$, may be accounted for on a similar footing to the geometrical symmetries. In particular, time reversal acts as the complex conjugate on the phase factors of the Bloch wavefunctions. This effect is the same as the $\hat{s}_{y}$ reflection, and therefore [38],

$$
\hat{T} H(\boldsymbol{r}) \equiv \sigma_{2} \tau_{2} H^{*}(\boldsymbol{r}) \tau_{2} \sigma_{2}
$$


Each of the $\sigma_{i}$ and $\tau_{i}$ separately are odd under time reversal.

Time reversal symmetry is obeyed quite generally in the absence of external magnetic fields. In the absence of an intervalley coupling (such as might be provided by a perturbation with the periodicity of the tripled graphene unit cell) the valley index $K_{ \pm}$is a good quantum number. Time reversal then allows the spectra in graphene's two valleys to be related ${ }^{\ddagger}$,

$$
\epsilon_{\boldsymbol{K}_{+}}(\boldsymbol{k})=\epsilon_{\boldsymbol{K}_{-}}(-\boldsymbol{k})
$$

\subsection{Coupling to the Electromagnetic Field}

The electromagnetic field is introduced into the Dirac Hamiltonian (Eq. (1.3)) using the Peierls substitution,

$$
H(\boldsymbol{p}) \rightarrow H(\boldsymbol{p}+e \boldsymbol{A}(\boldsymbol{r}, t))=v[\boldsymbol{p}+e \boldsymbol{A}(\boldsymbol{r}, t)] \cdot \boldsymbol{\sigma}
$$

where $\boldsymbol{A}(\boldsymbol{r}, t)$ is the vector potential, $e>0$, and we write $\boldsymbol{\sigma}=\left(\sigma_{1}, \sigma_{2}\right)$. The Hamiltonian for the coupling is then immediately written as,

$$
H_{e-e m}=\boldsymbol{A}(\boldsymbol{r}, t) \cdot \hat{\boldsymbol{j}}, \quad \hat{\boldsymbol{j}}=e \frac{\partial H_{D}(\boldsymbol{p})}{\partial \boldsymbol{p}}=e v \boldsymbol{\sigma} .
$$

For the interaction with photons, the electromagnetic field is quantised as [39],

$$
\boldsymbol{A}(\boldsymbol{r}, t)=\sum_{q, \boldsymbol{l}_{\boldsymbol{q}}} \frac{1}{\sqrt{2 \epsilon_{0} V \omega(\boldsymbol{q})}}\left(\boldsymbol{l} \hat{a}_{\boldsymbol{q}, l} e^{i(\boldsymbol{q} \cdot \boldsymbol{r}-\omega(\boldsymbol{q}) t)}+\boldsymbol{l}^{*} \hat{a}_{\boldsymbol{q}, l}^{\dagger} e^{-i(\boldsymbol{q} \cdot \boldsymbol{r}-\omega(\boldsymbol{q}) t)}\right)
$$

where $V$ is a 3 dimensional normalisation volume, $l$ the in-plane component of the polarisation, $\hat{a}_{\boldsymbol{q}, \boldsymbol{l}}$ the photon annihilation operator, and I have neglected to include the out of plane momentum. Also, as a matter of course, the momentum of the photon will be neglected as compared to that of the electronic states.

\footnotetext{
${ }^{\ddagger}$ Spatial inversion symmetry leads to the same relation.
} 


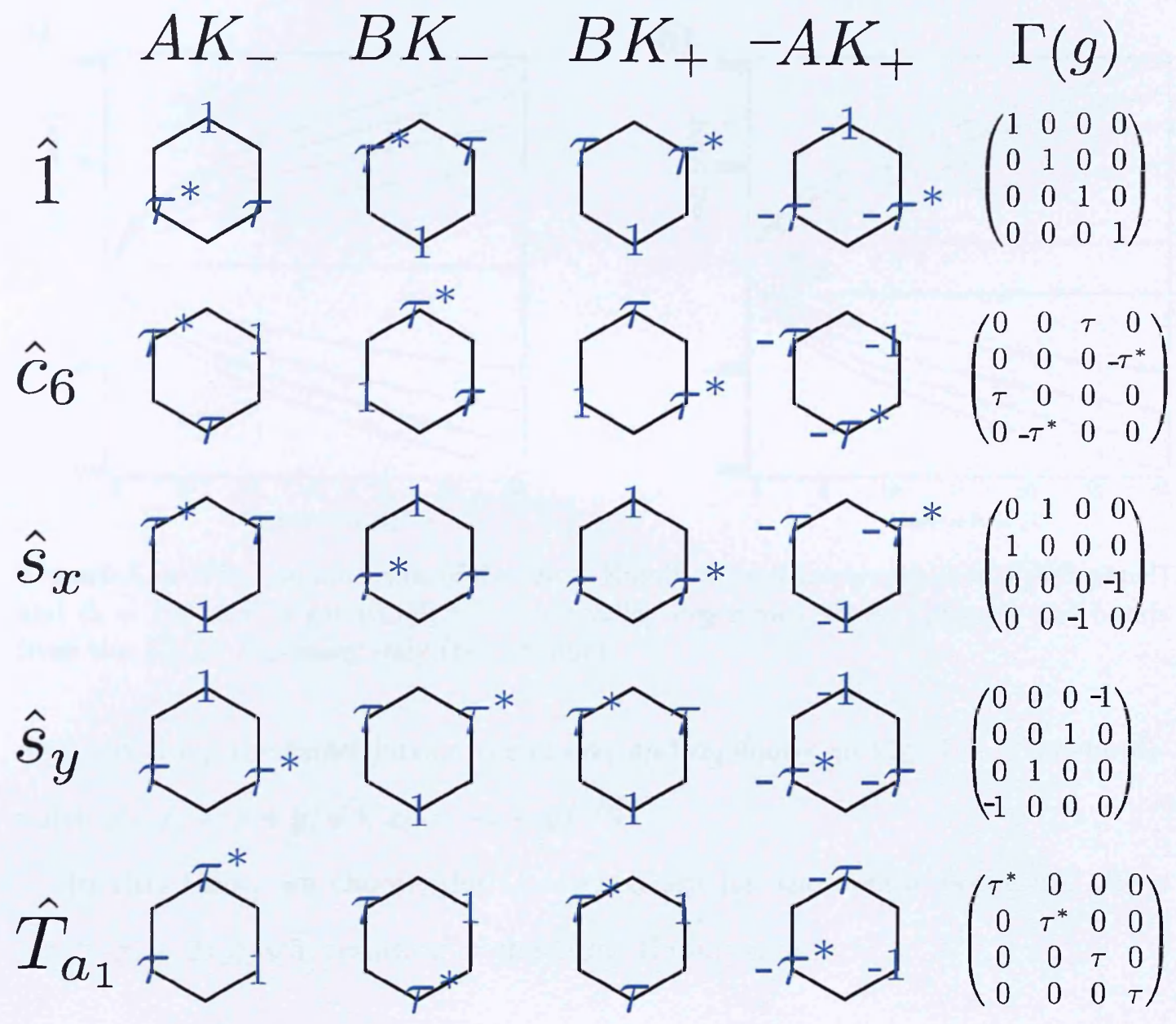

Figure 1.3: The transformation of the Bloch wavefunctions (1.2) upon application of symmetry operations from $c_{6 v^{\prime \prime}}$. The six corners of each hexagon represent the six atomic sites in the tripled unit cell. The top row shows the phases that the four Bloch wavefunctions, $\Phi_{i=\mathrm{A} / \mathrm{B} K_{ \pm}}$, place on each of these sites. Here $\tau \equiv e^{i 2 \pi / 3}$. The remaining rows show the phases for Bloch wavefunctions that have been affected by the symmetry transformations $\hat{c}_{6}, \hat{s}_{x}, \hat{s}_{y}, \hat{T}_{a_{1}}$. The matrix, $\Gamma(G)$, for each transformation is shown in the right column, and is deduced by comparing the transformed Bloch wavefunctions to their un-transformed counterparts in the top row.

\subsection{Landau Levels Spectra}

With a view to application in chapter 4, we now introduce the Landau levels [40] of graphene with a finite mass term. Here we use a non-orthogonal coordinate system adapted to the hexagonal symmetry of the graphene lattice. That is, we write $\boldsymbol{r}=x_{1} \hat{x}_{1}+x_{2} \hat{x}_{2}$, with basis vectors,

$$
\hat{x}_{1}=\frac{1}{2} \hat{x}+\frac{\sqrt{3}}{2} \hat{y}, \quad \hat{x}_{2}=-\frac{1}{2} \hat{x}+\frac{\sqrt{3}}{2} \hat{y}
$$


a)

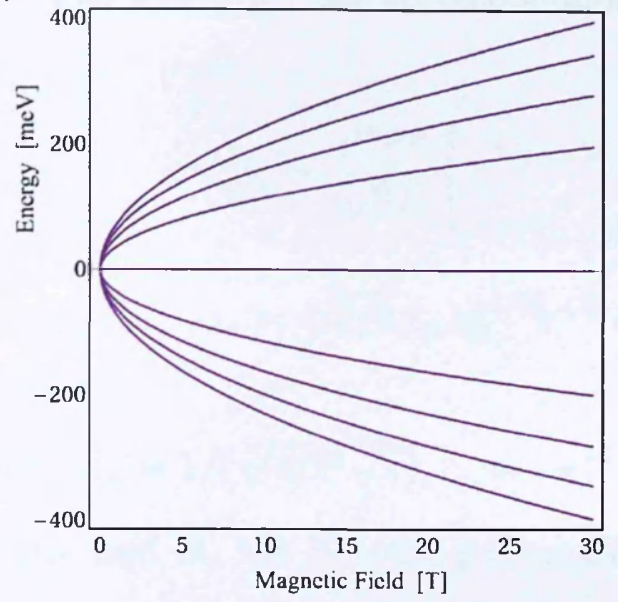

b)

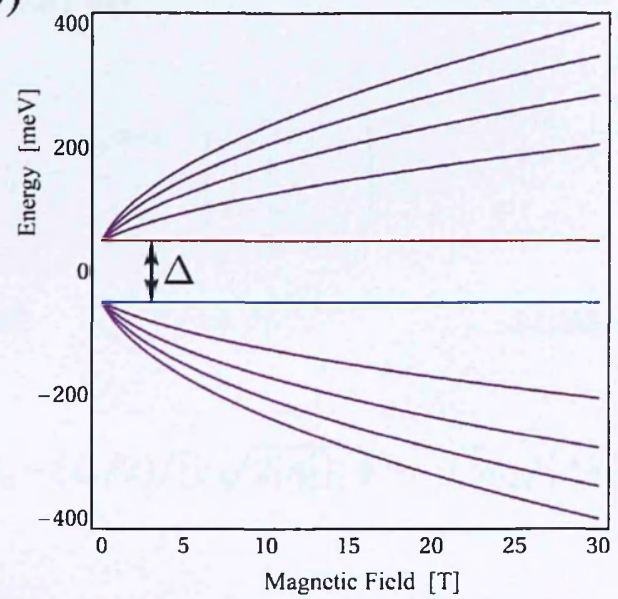

Figure 1.4: The Landau levels of the Dirac Hamiltonian, shown with $\Delta=0$ (left panel) and $\Delta=100 \mathrm{meV}$ (right panel). Showing valley degenerate bands (purple) and bands from the $K_{+}$or $K_{-}$valley only (red or blue).

directed along the moiré lattice vectors $\boldsymbol{a}_{1}$ and $\boldsymbol{a}_{2}$ shown in Fig. 1.1. The coordinates are $x_{1}=x+y / \sqrt{3}, x_{2}=-x+y / \sqrt{3}$.

In this basis, we choose the Landau gauge for the vector potential, $\boldsymbol{A}=$ $B x_{1}\left(-\hat{x}_{1}+2 \hat{x}_{2}\right) / \sqrt{3}$, resulting in the Dirac Hamiltonian,

$$
H_{D}=\left(\begin{array}{cc}
1 / 2 \tau_{z} \Delta & v d^{\dagger} \\
v d & -1 / 2 \tau_{z} \Delta
\end{array}\right), \quad d=\frac{-2}{\sqrt{3}}\left[\partial_{x_{1}} e^{i \frac{2 \pi}{3}}+\left(\partial_{x_{2}}+i \sqrt{3} e B x_{1} / 2\right) e^{-i \frac{2 \pi}{3}}\right] .
$$

Here Eq. (1.18) has been used to introduce the magnetic field. The gap, $\Delta$, is reflected in the resulting Landau level spectrum when a magnetic field is applied,

$$
E_{n \neq 0}=\operatorname{sign}(n) \sqrt{\Delta^{2} / 4+2|n| v^{2} / \lambda_{B}^{2}}, \quad E_{0}=\frac{\xi}{2} \frac{B}{|B|} \Delta
$$

where $\xi= \pm 1$ for the $K_{ \pm}$valleys, and $\lambda_{B}=1 / \sqrt{|e B|}$ is the magnetic length. For $\Delta=0$, the Landau levels fan out with the peculiar $\epsilon \sim \pm \sqrt{n B}$ dependence $[35,41,42]$ shown in Fig. 1.4a. A finite gap, $\Delta \neq 0$, will manifest itself as a gapped Landau level sequence, and broken valley degeneracy for the zeroth Landau level (Fig. 1.4b). The latter is possible due to the absence of both time-reversal and spatial-inversion symmetries in $H_{D}$. 
The wavefunctions corresponding to Eq. (1.22) are,

$$
\begin{aligned}
& \psi_{n \neq 0}^{k_{2}}=\frac{e^{i k_{2} x_{2}}}{\sqrt{2 L}}\left(\begin{array}{c}
\varphi_{|n|+\frac{s_{B}-1}{2}} \\
c_{n} \varphi_{|n|-\frac{s_{B}+1}{2}}
\end{array}\right), \quad \psi_{0}^{k_{2}}=\frac{e^{i k_{2} x_{2}}}{\sqrt{L}}\left(\begin{array}{c}
\frac{1+s_{B}}{2} \varphi_{0} \\
\frac{1-s_{B}}{2} \varphi_{0}
\end{array}\right), \\
& \varphi_{n}=\sqrt{3 / 2} A_{n} \lambda_{B}^{-1 / 2} e^{-\frac{z^{2}}{2}} e^{-\frac{i z^{2}}{2 \sqrt{3}}} \mathbb{H}_{n}(z) .
\end{aligned}
$$

Here $A_{n}=1 /\left(\sqrt{n ! 2^{n} \sqrt{\pi}}\right), c_{n}=-e^{-i \frac{2 \pi}{3}} s_{B} \lambda_{B}\left(E_{n}-\xi \Delta / 2\right) /(v \sqrt{2|n|}), z=\sqrt{3} x_{1} /\left(2 \lambda_{B}\right)-$ $k_{2} \lambda_{B}$, and $\mathbb{H}_{n}$ the Hermite polynomial.

\subsection{Spectra of Bilayer Graphene}

a)



b)

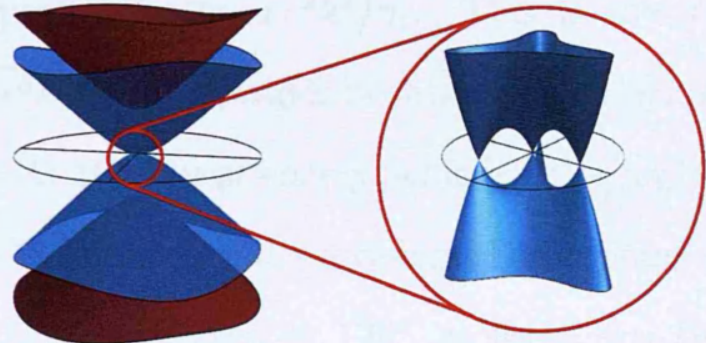

Figure 1.5: a) The crystal structure of $A-B$ stacked bilayer graphene, showing the nearest neighbour intralayer coupling, $\gamma_{0}$, and interlayer couplings $\gamma_{1}$ and $\gamma_{3}$. b) The corresponding bandstructure with an enlargement of the low-energy bands.

The tight binding model of section 1.3 can be adapted for bilayer graphene. Here we consider $A-B$ stacked bilayer [42], shown in Fig. 1.5a, which consists of two parallel graphene layers, stacked such that the $A$ site of the top layer sits directly above the $B$ site of the bottom layer. Here we only take into account the two most important interlayer couplings, the vertical hop $\gamma_{1} \approx 0.39 \mathrm{eV}$ and the skew hop $\gamma_{3} \approx 0.32 \mathrm{eV}$, as well as the intralayer $\gamma_{0} \approx 3.0 \mathrm{eV}$ [35]. We immediately concentrate on the vicinity of the Brillouin zone corners, and write the momentum $\boldsymbol{p}$ with respect to the valley centre,

$$
\hat{H}^{\mathrm{BLG}}=\left(\begin{array}{cc}
v_{3}(\boldsymbol{\sigma} \cdot \boldsymbol{p})^{T} & v \boldsymbol{\sigma} \cdot \boldsymbol{p} \\
v \boldsymbol{\sigma} \cdot \boldsymbol{p} & \gamma_{1} \sigma_{1} \tau_{3}
\end{array}\right) ; \quad v=\frac{\sqrt{3}}{2} a \gamma_{0}, \quad v_{3}=\frac{\sqrt{3}}{2} a \gamma_{3}, \quad v_{3} \ll v
$$


The Hamiltonian is written in the basis of Bloch states $\left(\Phi_{A 1}, \Phi_{B 2}, \Phi_{A 2}, \Phi_{B 1}\right)$ for the $K_{+}$valley or $\left(\Phi_{B 2},-\Phi_{A 1}, \Phi_{B 1},-\Phi_{A 2}\right)$ for the $K_{-}$valley. The bandstructure of Hamiltonian (1.24) contains a pair of high-energy split bands (shown in red in Fig. 1.5b) with a dispersion, $\epsilon \approx \pm\left(\gamma_{1}+\sqrt{4 v^{2} k^{2}+\gamma_{1}^{2}}\right) / 2$, that is approximately quadratic for $v p \ll \gamma_{1}$. To discuss the low-energy bands, it is convenient to perform the Schrieffer-Wolff transformation [43], projecting onto the low-energy $2 \times 2$ subspace in the top right hand corner of (1.24),

$$
\hat{H}_{2 \times 2}^{\mathrm{BLG}}=-\frac{v^{2}}{\gamma_{1}}\left[\left(p_{x}^{2}-p_{y}^{2}\right) \sigma_{1}+2 p_{x} p_{y} \sigma_{2}\right] \tau_{z}+v_{3}(\boldsymbol{p} \cdot \boldsymbol{\sigma})^{T}
$$

For the intermediate energy scale $\gamma_{1}\left(v_{3} / v\right)^{2} \ll|\epsilon| \ll \gamma_{1}$ the low-energy bands obtained from Hamiltonian (1.25) disperse as $\epsilon \approx \pm v^{2} k^{2} / \gamma_{1}$. This is the low energy approximation of $\epsilon \approx \pm\left(\gamma_{1}-\sqrt{4 v^{2} k^{2}+\gamma_{1}^{2}}\right) / 2$, which is obtained for the lowenergy bands from Hamiltonian (1.24). At the lowest energy scale $|\epsilon|<\gamma_{1}\left(v_{3} / v\right)^{2}$ the spectra is trigonally warped by the $v_{3}$ term, forming a central Dirac point at $\boldsymbol{k}=0$ with three surrounding Dirac points, separated by $120^{\circ}$, at $|\boldsymbol{k}|=\gamma_{1} v_{3} / v^{2}$. This shown by the inset in Fig. 1.5b.

\subsection{Hexagonal Boron Nitride}

Hexagonal Boron Nitride (hBN) is becoming the substrate of choice for graphene devices. The relative smoothness of the surface of this material, combined with the reduced density of charged impurities relative to other substrates, means that the charge fluctuations (electron-hole puddles) found in graphene on this material are substantially reduced [9-15].

HBN has the same layered honeycomb structure as graphene (shown in Fig. 1.1), but whereas for graphene both the $A$ and $B$ sites are occupied by carbon atoms, in $\mathrm{hBN}$ the $A$ site (say) contains a nitrogen atom while the $B$ site contains the boron atom. The lattice constant is also very similar to graphene, being only $1.8 \%$ larger. Chemically the nitrogen atom, having an atomic number of 7 , and boron, 
having an atomic number of 5 , sit on either side of carbon (atomic number 6 ) in the Periodic Table. Therefore the electronic properties of hBN may be modelled in a similar manner to that of graphene, the difference arising only from the fact that the two atoms in the unit cell are not equivalent. Within the tight binding model (section. 1.3) this difference is included by using different on-site energies, $E_{N}$ and $E_{B}$, for the $2 p_{z}$ orbitals of nitrogen and boron. This, in turn, leads to a gapped Dirac Hamiltonian, for the Brillouin zone corners of hBN,

$$
H_{B N}=v_{B N} \boldsymbol{p} \cdot \boldsymbol{\sigma}+\frac{\Delta_{B N}}{2} \sigma_{3} \tau_{3}
$$

with $v_{B N}$ the Dirac velocity appropriate for $\mathrm{hBN}$ and $\Delta_{B N}=\left(E_{N}-E_{B}\right) \approx$ $4.6 \mathrm{eV}[44]$. The resulting dispersion relation, $\epsilon= \pm \sqrt{v_{B N}^{2} k^{2}+\left(\Delta_{B N} / 2\right)^{2}}$ indicates that $\mathrm{hBN}$ is an insulator with a large band gap. The second term in Eq. (1.26), which is responsible for the band gap, anticommutes with the matrix for inversion symmetry, (1.14), and is therefore forbidden in the Hamiltonian for graphene.

\subsection{The Moiré Pattern}

The term moiré pattern is used to describe the beating of any two short range periodic patterns to create a single long range quasi-periodic pattern. Moiré patterns are found in everyday settings, for example when a line of sight intersects two similar fences; technological settings $[45,46]$, including strain measurements, and range sensing (where the moiré effect is useful) or image processing and under-sampling (where it produces unwanted artifacts).

For the purpose of this Thesis, we are primarily interested in the moiré patterns that arise when graphene is placed on top of a crystal whose top layer of atoms has the hexagonal Bravais lattice and a lattice constant similar to graphene, e.g.. top row of Fig. 1.6. The hBN substrate is the archetypal example, with other examples including $\operatorname{Ir}(111), \mathrm{Ni}(111), \mathrm{Cu}(111), \mathrm{Rh}(111), \mathrm{Ru}(0001)$ and $\mathrm{Pt}(111)$ [48]. In chapter 7 , we also consider underlays which are almost commensurate with the 
$\sqrt{3} \times \sqrt{3}$ graphene superlattice. Here, however, I shall give a more general account of this geometry, applicable to any two similar crystal lattices.

Suppose two crystals are placed on top of each other, the top crystal having primitive lattice vectors $\boldsymbol{a}_{i}$ and reciprocal lattice vectors $\boldsymbol{g}_{i}$, and the underlay with primitive lattice vectors $\boldsymbol{a}_{i}^{\prime}=(1+\delta) \hat{R}_{\theta} \boldsymbol{a}_{i}$ and reciprocal lattice vectors $\boldsymbol{g}_{i}^{\prime}=$ $(1+\delta)^{-1} \hat{R}_{\theta} \boldsymbol{g}_{i}$. Here $\delta, \theta \ll 1$ are the lattice mismatch and misalignment angle and $\hat{R}_{\theta}$ is the anticlockwise rotation matrix. The reciprocal lattice vectors of the moiré pattern, $\boldsymbol{G}_{i}$, can then be written immediately,

$$
\boldsymbol{G}_{i}=\boldsymbol{g}_{i}-\boldsymbol{g}_{i}^{\prime}=W_{\delta,-\theta} \boldsymbol{g}_{i}, \quad W_{\delta,-\theta}=\left(1-(1+\delta)^{-1} \hat{R}_{\theta}\right) \approx\left(\begin{array}{cc}
\delta & \theta \\
-\theta & \delta
\end{array}\right)
$$

where $\theta, \delta \ll 1$ has been assumed in the approximation of $W_{\delta,-\theta}$. In order to find the primitive moiré lattice vectors, $\boldsymbol{A}_{i}$, we use the definition, $\boldsymbol{A}_{i} \cdot \boldsymbol{G}_{j}=2 \pi \delta_{i j}$, resulting in,

$$
\boldsymbol{A}_{i}=W_{\delta, \theta}^{-1} \boldsymbol{a}_{i}, \quad\left|\boldsymbol{A}_{i}\right| \approx \frac{\left|\boldsymbol{a}_{i}\right|}{\sqrt{\delta^{2}+\theta^{2}}}
$$

It should be noted that the moiré lattice is not, in general, the same as the crystallographic lattice of the two layer system. The crystallographic lattice is defined in terms of a perfect translational symmetry that is only present when the periodicities of the two layers are commensurate. In contrast, moiré lattice vectors (1.28) are always defined. Moreover, for $\left|\boldsymbol{A}_{i}\right| \gg\left|\boldsymbol{a}_{i}\right|$ limit, it is natural to expect that it will be the moiré periodicity that determines the physical properties of the system, rather than the true crystallographic periodicity.

It is also interesting to note that if the underlay undergoes a translation $\boldsymbol{u}$, the moiré pattern will undergo the larger translation [47] $\boldsymbol{U}=-W_{\delta,-\theta}^{-1} \boldsymbol{u}$. This effect has been used to measure the radius of curvature of the graphene over the step edges on $\operatorname{Ir}(111)$ surfaces [49]. Due to its Face Centred Cubic crystal structure, there is an in-plane displacement between atomic positions in different atomic 


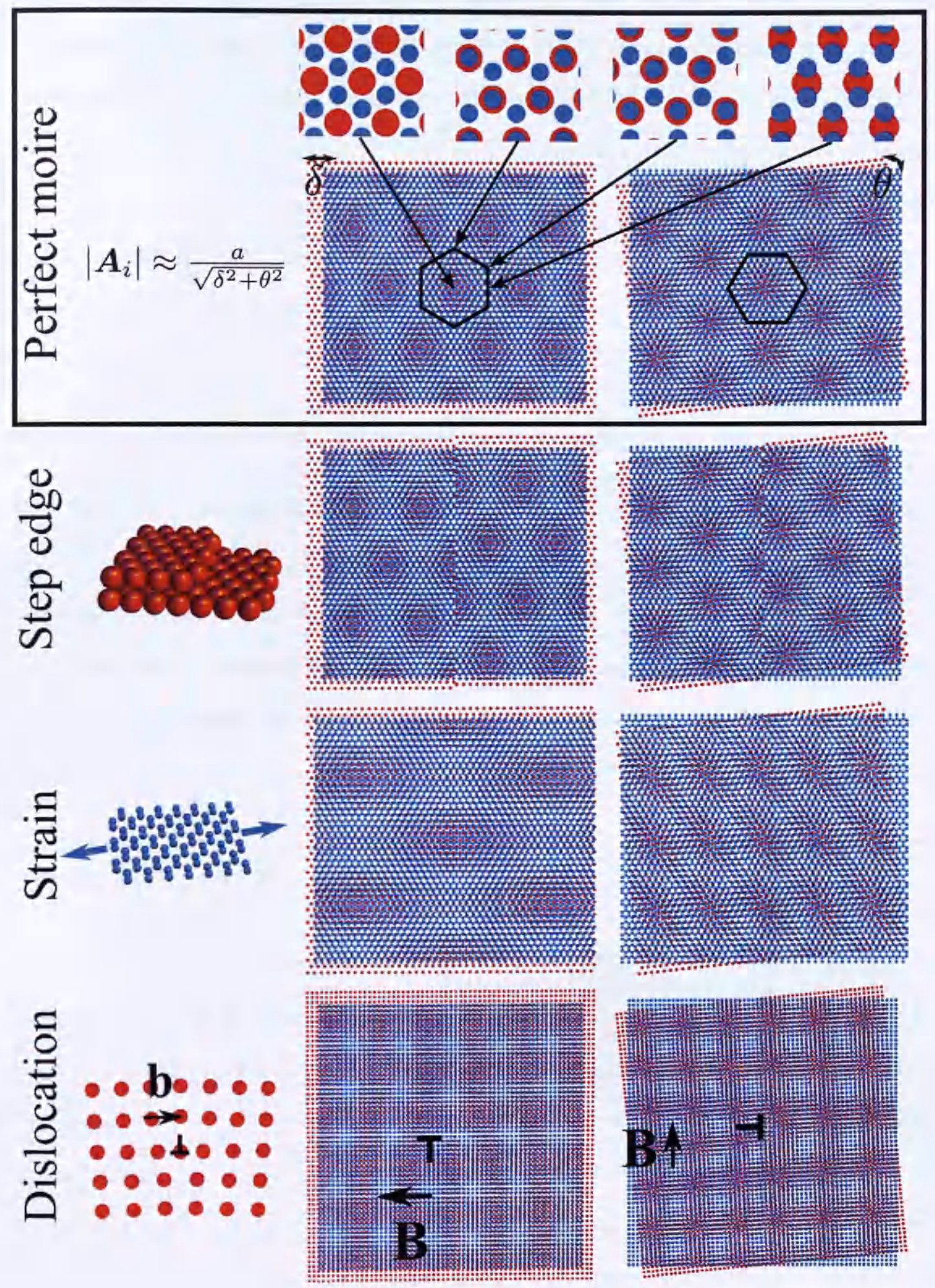

Figure 1.6: Top row: The defect-free moiré pattern of graphene (blue) on a simple hexagonal underlay (red), created by either a lattice mismatch (left), or rotational misalignment (right). Enlargements show the local atomic arrangements at different positions with in the moiré unit cell. Lower rows: The magnifying effect that the moiré pattern has on various types of defect. In the bottom panels the square lattice is used for clarity. 
layers. Moreover, well aligned graphene grows continuously over the step edge to produce a displacement in the moiré pattern, which, due to the large scale of the moiré pattern can be easily measured. This effect is shown in the second row of Fig. 1.6. The effect of a small uniform strain, applied to the overlay, may also be accounted for using the frame-work of this section. This has the dramatic effect on the moiré pattern shown in the third row Fig. 1.6, and illustrates the moiré magnifying effect discussed more generally in the next section.

\subsection{The Moiré Magnifying Glass}

In this section we describe the magnifying effect that the moiré pattern has on defects in the underlay (see Fig. 1.6). However, Eq. (1.28) is only applicable to the superposition of two perfect crystals, and so we begin by rigorously defining the moiré lattice. Suppose $H(\boldsymbol{r})$ is a scalar function, e.g. the height of the overlay, which depends smoothly on the relative local displacements of atoms in the two layers. This function can written using a periodic function, $h(\boldsymbol{r})$,

$$
H(\boldsymbol{r})=h(\boldsymbol{u}(\boldsymbol{r})), \quad h\left(\boldsymbol{r}+\boldsymbol{a}_{i}\right)=h(\boldsymbol{r}) .
$$

Here $\boldsymbol{u}(\boldsymbol{r})$ is the relative displacement of atoms in the second layer, with respect to the perfect reference lattice, in which every underlay atom sits directly underneath its counter part in layer one. It is assumed that this relative displacement varies smoothly over the scale of many lattice periods, $\boldsymbol{a}_{i}$, which allows the continuous function $\boldsymbol{u}(\boldsymbol{r})$ to be defined unambiguously ${ }^{\S}$. Note $\boldsymbol{u}(\boldsymbol{r})$ depends on both the lattice mismatch and misalignment angle, in addition to the displacement fields of defects in the underlay. The moiré spots, which define the moiré superlattice, are defined by the maxima of $H(\boldsymbol{r})$. These are assumed to occur at each position

\footnotetext{
$\S_{\text {Note }} \boldsymbol{u}(\boldsymbol{r})$ describes the vector by which a patch of material at position $\boldsymbol{r}$, in the already displaced second layer, was displaced by. This contrasts with the usual definition, $\boldsymbol{u}^{f}(\boldsymbol{r})$, which describes the vector by which a patch of material at $r$, in the un-displaced second layer, will be displaced. The two displacements are related by $\boldsymbol{u}(\boldsymbol{r})=\boldsymbol{u}^{f}\left(\boldsymbol{r}-\boldsymbol{u}^{f}(\boldsymbol{r})\right)$, and error caused by neglecting this subtlety is on the atomic scale, which is much smaller than the moire scale by assumption.
} 
$\boldsymbol{r}=\boldsymbol{A}_{m n}$ such that,

$$
\boldsymbol{u}\left(\boldsymbol{A}_{m n}\right)=m \boldsymbol{a}_{1}+n \boldsymbol{a}_{2}, \quad n, m \text { integer. }
$$

The utility of this approach is illustrated by the example of a dislocation in the underlay. A dislocation [50] creates a field of displacements $\boldsymbol{u}_{d}(\boldsymbol{r})$ with a non-zero Burgers vector, $\boldsymbol{b}$,

$$
\boldsymbol{b}=\oint d s \frac{d \boldsymbol{u}_{d}(\boldsymbol{r}(s))}{d s}
$$

Qualitatively, the burgers vector characterises the number of rows of atoms that have been removed from the lattice (see bottom left panel of Fig. 1.6). Given a dislocation in the underlay, with burgers vector $\boldsymbol{b}$, we wish to calculate the corresponding burgers vector, $\boldsymbol{B}$, in the moiré pattern. Suppose that the displacement field is,

$$
\boldsymbol{u}(\boldsymbol{r})=W_{\delta, \theta} \cdot \boldsymbol{r}+\boldsymbol{u}_{d}(\boldsymbol{r})
$$

The first term Eq. (1.32) accounts for the lattice mismatch and misalignment angle, with $W_{\delta, \theta}$ given in Eq. (1.27), whereas the second term accounts for the dislocation. To calculate the displacement of the moiré lattice, $\boldsymbol{U}^{d} \equiv \boldsymbol{A}_{m n}^{d}-\boldsymbol{A}_{m n}$, describing the position of the moiré spot in the presence of the dislocation, $\boldsymbol{A}_{m n}^{d}$, relative to its position in the absence of the dislocation, $\boldsymbol{A}_{m n}$, we use definition (1.30),

$$
\begin{aligned}
& W_{\delta, \theta} \cdot \boldsymbol{A}_{m n}=m \boldsymbol{a}_{1}+n \boldsymbol{a}_{2} \\
& W_{\delta, \theta} \cdot \boldsymbol{A}_{m n}^{d}+\boldsymbol{u}_{d}\left(\boldsymbol{A}_{m n}^{d}\right)=m \boldsymbol{a}_{1}+n \boldsymbol{a}_{2},
\end{aligned}
$$

This gives,

$$
\boldsymbol{U}^{d}=\boldsymbol{A}_{m n}^{d}-\boldsymbol{A}_{m n}=-W_{\delta, \theta}^{-1} \boldsymbol{u}_{d}\left(\boldsymbol{A}_{m n}^{d}\right) .
$$


The linear transformation, $-W_{\delta, \theta}^{-1}$, describing the relation of $\boldsymbol{U}^{d}$ to $\boldsymbol{u}_{d}$ can be taken through the integration in the definition of the Burgers vector, Eq. (1.31), to obtain,

$$
\boldsymbol{B}=-W_{\delta, \theta}^{-1} \boldsymbol{b}
$$

That is, each dislocation in the underlay gives rise to a partner dislocation in the moiré pattern, but with a Burgers vector that depends on the lattice mismatch and misalignment angle. This is clearly seen in the bottom row of Figure 1.6. 


\section{Chapter 2}

\section{Monolayer Graphene on a hBN}

\section{underlay}

\section{$2.1 \quad$ Introduction}

It has been demonstrated that the electronic quality of graphene-based devices can be dramatically improved by placing graphene on an atomically flat crystal surface, such as hexagonal boron nitride (hBN) [9-15]. At the same time, graphene's electronic spectrum also becomes modified, acquiring a complex, energy-dependent form caused by incommensurability between the graphene and substrate crystal lattices $[18,19,22]$. For graphene placed on hBN, the moire pattern $[10,11,18,19$, 22 creates a periodic perturbation, usually referred to as a superlattice, which acts on graphene's charge carriers and leads to multiple minibands [20] and the generation of secondary Dirac-like spectra. The resulting new Dirac fermions present yet another case where graphene allows mimicking of QED phenomena under conditions that cannot be achieved in particle physics experiments. In contrast to relativistic particles in free space, the properties of secondary Dirac fermions in graphene can be affected by a periodic sublattice symmetry breaking and modulation of carbon-carbon hopping amplitudes, in addition to a simple potential modulation. The combination of different features in the modulation results in 

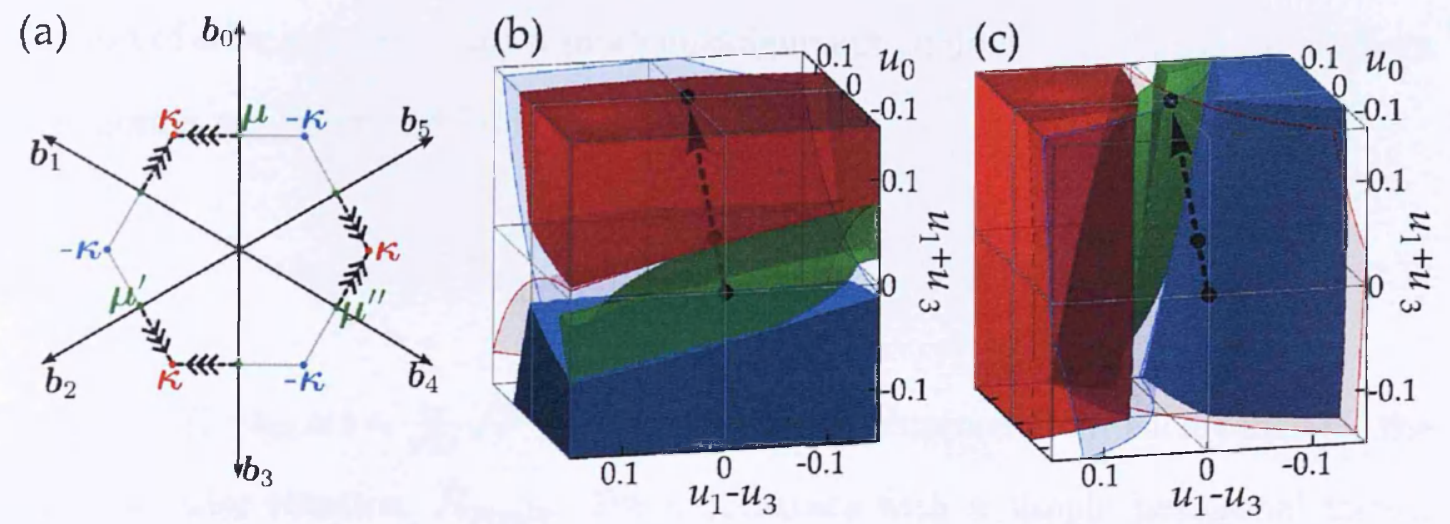

Figure 2.1: (a) The hexagonal Brillouin zone for the moire superlattice. (b) Three volumes in the space of the moiré superlattice parameters where the edge of the first miniband, in graphene's valence band, contains an isolated sDP at the $\kappa$-point (red) or the $-\kappa$-point (blue) or three isolated sDPs at the sBZ edge (green). Parameters for which the $\pm \kappa$-point is triple degenerate are shown by the red and blue surfaces. The black dots represent sets of perturbation parameters for which miniband spectra are shown in Fig. 2.2. (c)The same for the conduction band in graphene.

a multiplicity of possible outcomes for the moiré miniband spectrum in graphene which we systematically investigate in this chapter.

\subsection{Superlattice Hamiltonian}

To describe the effect of a substrate on electrons in graphene at a distance, $d$, much larger than graphene's lattice constant, $a$, we use the earlier observation $[18,19,22,51-54]$ that, at $d \gg a$, the lateral variation of the wavefunctions of the $\mathrm{p}^{\mathrm{z}}$ carbon orbitals is smooth on the scale of $a$. This is manifested in the comparable sizes of the skew and vertical hopping in graphite and permits an elegant continuum-model description [51-54] of the interlayer coupling in twisted bilayers and the resulting band structure. A similar idea applied to graphene on a hBN substrate $[18,19,22]$ suggests that a substrate perturbation for Dirac. electrons in graphene can be described in terms of simple harmonic functions corresponding to the six smallest reciprocal lattice vectors of the moiré superlattice.

Below, we shall use a similar approach to analyse the generic properties of moiré minibands for electrons in graphene subjected to a substrate with a hexagonal Bravais lattice with a slightly different lattice constant of $(1+\delta) a,|\delta| \ll 1$, compared 
to that of $a$ for graphene, and a small misalignment angle, $\theta \ll 1$. The moiré pattern harmonics are described by vectors

$$
\boldsymbol{b}_{m=0, \cdots 5}=\hat{R}_{\frac{2 \pi m}{6}} \boldsymbol{b}_{0}, \quad \boldsymbol{b}_{0}=\left[1-(1+\delta)^{-1} \hat{R}_{\theta}\right]\left(0, \frac{4 \pi}{\sqrt{3} a}\right)
$$

with length $\left|\boldsymbol{b}_{0}\right| \equiv b \approx \frac{4 \pi}{\sqrt{3} a} \sqrt{\delta^{2}+\theta^{2}}$, which can be obtained from each other by the anticlockwise rotation, $\hat{R}_{2 \pi m / 6}$. For a substrate with a simple hexagonal lattice or a honeycomb lattice with two identical atoms, the perturbation created for graphene electrons is inversion-symmetric. For a honeycomb substrate where one of the atoms would affect graphene electrons stronger than the other (e.g. such as $\mathrm{hBN}$, for which the occupancy and size of the $\mathrm{p}^{\mathrm{z}}$ orbitals are different) the moiré potential can be modelled as a combination of a dominant inversion-symmetric part with the addition of a small inversion-asymmetric perturbation,

$$
\begin{aligned}
\hat{H}=v \boldsymbol{p} \cdot \boldsymbol{\sigma} & +u_{0} v b f_{1}(\boldsymbol{r})+u_{3} v b f_{2}(\boldsymbol{r}) \sigma_{3} \tau_{3}+u_{1} v\left[\boldsymbol{l}_{z} \times \nabla f_{2}(\boldsymbol{r})\right] \cdot \boldsymbol{\sigma} \tau_{3}+u_{2} v \nabla f_{2}(\boldsymbol{r}) \cdot \boldsymbol{\sigma} \tau_{3} \\
& +\tilde{u}_{0} v b f_{2}(\boldsymbol{r})+\tilde{u}_{3} v b f_{1}(\boldsymbol{r}) \sigma_{3} \tau_{3}+\tilde{u}_{1} v\left[\boldsymbol{l}_{z} \times \nabla f_{1}(\boldsymbol{r})\right] \cdot \boldsymbol{\sigma} \tau_{3}+\tilde{u}_{2} v \nabla f_{1}(\boldsymbol{r}) \cdot \boldsymbol{\sigma} \tau_{3} .
\end{aligned}
$$

The Hamiltonian, $\hat{H}$, is written in terms of direct products $\sigma_{i} \tau_{j}$, of the Pauli matrices described in section 1.5.2. The first term in $\hat{H}$ is the Dirac part, with $\boldsymbol{p}=-i \nabla+e \boldsymbol{A}$ describing the momentum relative to the centre of the corresponding valley, with $\nabla \times \boldsymbol{A}=B$. The rest of the first line in Eq. (2.2) describes the inversion-symmetric part of the moiré perturbation, whereas the second line takes into account its inversion-asymmetric part. In the first line, the first term, with $f_{1}(\boldsymbol{r})=\sum_{m=0 \ldots 5} e^{i \boldsymbol{b}_{m} \cdot \boldsymbol{r}}$, describes a simple potential modulation. The second term, with $f_{2}(\boldsymbol{r})=i \sum_{m=0 \ldots 5}(-1)^{m} e^{i \boldsymbol{b}_{m} \cdot \boldsymbol{r}}$, accounts for the $A-B$ sublattice asymmetry, locally imposed by the substrate. The third term, with unit vector $\boldsymbol{l}_{z}$, describes the influence of the substrate on the $A-B$ hopping: consequently [55-57], this term can be associated with a pseudo-magnetic field, $e B^{\mathrm{eff}}= \pm u_{1} b^{2} f_{2}(\boldsymbol{r})$, which has opposite signs in valleys $K_{ \pm}$. Each of the coefficients $\left|u_{i}\right| \ll 1$ in Eq. (2.2) is a dimensionless 
phenomenological parameter with the energy scale set by $v b \approx 2 \pi \sqrt{\delta^{2}+\theta^{2}} \gamma_{0}$, where $\gamma_{0} \approx 3 \mathrm{eV}$ is the nearest neighbour hopping integral in the SlonczewskiWeiss tight binding model [3]. Concerning the inversion-asymmetric part, the second line in Eq. (2.2), we assume that $\left|\tilde{u}_{i}\right| \ll\left|u_{i}\right|$. Note that the last term in each line can be gauged away using $\boldsymbol{\psi} \rightarrow e^{-i \tau_{3}\left(u_{2} f_{2}+\tilde{u}_{2} f_{1}\right)} \boldsymbol{\psi}$.

Hamiltonian, $\hat{H}$, may be used to parametrise any microscopic model compatible with the symmetries of the system (see section 2.5) and the dominance of the simplest moiré harmonics, $e^{i \boldsymbol{b}_{m} \cdot \boldsymbol{r}}$, in the superlattice perturbation. The values that parameters $u_{i}$ take are listed in Table 2.1 for several models of graphene on an hBN substrate, both taken from the recent literature $[18,19,22]$ and analysed in sections 2.6.1 and 2.6.2, including a simple model in which the hBN substrate is treated as a lattice of positively charged nitrogen nuclei with a compensating homogeneous background of electron $\mathrm{P}^{\mathrm{z}}$ orbitals. The examples of model-dependent values of parameters $u_{i}$, listed in Table 2.1, indicate that the combination of several factors can strongly shift the resulting moiré perturbation across the parameter space in Fig. 2.1. That is why, in this chapter, we analyse the generic features of the miniband spectra generated by the moiré superlattice, rather than attempt to make a brave prediction about its exact form for a particular substrate.

\begin{tabular}{|l|c|c|c|c|}
\hline Model & $\begin{array}{c}v b u_{0} \\
{[\mathrm{meV}]}\end{array}$ & $\begin{array}{c}v b u_{1} \\
{[\mathrm{meV}]}\end{array}$ & $\begin{array}{c}v b u_{2} \\
{[\mathrm{meV}]}\end{array}$ & $\begin{array}{c}v b u_{3} \\
{[\mathrm{meV}]}\end{array}$ \\
\hline Potential modulation [22] & 60 & 0 & 0 & 0 \\
\hline 2D charge modulation [18] & $-\frac{V_{0}}{2}$ & 0 & 0 & $\frac{\sqrt{3} V_{0}}{2}$ \\
\hline $\begin{array}{l}\text { One-site version of G-hBN } \\
\text { hopping [19] (section 2.6.2) }\end{array}$ & 1.6 & $\frac{-3.2 \delta}{\sqrt{\delta^{2}+\theta^{2}}}$ & $\frac{3.2 \theta}{\sqrt{\delta^{2}+\theta^{2}}}$ & -2.8 \\
\hline $\begin{array}{l}\text { Point charge lattice } \\
\text { (section 2.6.1), } 0.6 \leq \tilde{v} \leq 3.4\end{array}$ & $\frac{\tilde{v}}{2}$ & $\frac{-\tilde{v} \delta}{\sqrt{\delta^{2}+\theta^{2}}}$ & $\frac{\tilde{v} \theta}{\sqrt{\delta^{2}+\theta^{2}}}$ & $-\frac{\sqrt{3} \tilde{v}}{2}$ \\
\hline
\end{tabular}

Table 2.1: The inversion-symmetric parameters, $v b u_{i}$, for various models of the moiré superlattice. In the $2 \mathrm{D}$ charge modulation model [18], $V_{0}$ is a phenomenological parameter. The G-hBN hopping model in Ref. [19] used the hopping parameter from twisted bilayer graphene. Estimates in sections 2.6.1 and 2.6.2 show that the sets of parameters using a model of point charges attributed to nitrogen sites and for the G-hBN hopping model are very similar. 
In the absence of a magnetic field, the Hamiltonian Eq. (2.2) obeys timereversal symmetry, which follows from both $\sigma_{i}$ and $\tau_{i}$ changing sign upon the transformation $t \rightarrow-t$ (section 1.5.4). As a result, $\epsilon_{K_{+}+\boldsymbol{p}}=\epsilon_{\boldsymbol{K}_{--} \boldsymbol{p}}$ and we limit the discussion of minibands to the $K_{+}$valley. Subject to this limitation, the bandstructure for the inversion-symmetric superlattice perturbation obeys the $c_{3 v}$ symmetry. Moreover, using the commutation properties of $\sigma_{i}$ one can establish that

$$
\epsilon_{\boldsymbol{K}_{+}+\boldsymbol{p}}^{u_{0}, u_{1}, u_{3}}=-\epsilon_{\boldsymbol{K}_{+}-\boldsymbol{p}}^{-u_{0}, u_{1}, u_{3}}=-\epsilon_{\boldsymbol{K}_{+}+\boldsymbol{p}}^{-u_{0}, u_{1},-u_{3}}=\epsilon_{\boldsymbol{K}_{+}-\boldsymbol{p}}^{u_{0},-u_{1},-u_{3}}
$$

\subsection{Generic Miniband Spectra of Graphene-hBN \\ Heterostructures}

To calculate the miniband spectrum for $\hat{H}$ in Eq. (2.2) we perform zone folding (in the graphene $K_{+}$valley) bringing states with momenta related by the reciprocal lattice vectors $n_{1} \boldsymbol{b}_{1}+n_{2} \boldsymbol{b}_{2}$ of the moiré pattern to the same point of the superlattice Brillouin zone (sBZ) in Fig. 2.1(a). Then, we calculate the matrix elements of $\hat{H}$ between those states and diagonalise the corresponding Heisenberg matrix numerically exploring the parametric space $\left(u_{0}, u_{1}, u_{3}\right)$ of the dominant inversionsymmetric part of the moiré perturbation shown in Fig $2.1(b, c)$. The size of the matrix is chosen to guarantee the convergence of the calculated energies for the three lowest minibands in both the conduction band $(s=+1)$ and the valence band $(s=-1)$. Below, we discuss the generic features of the moiré miniband spectra for the characteristic points in the parametric space $\left(u_{0}, u_{1}, u_{3}\right)$, marked using black dots in Fig. 2.1(b,c), using both the numerically calculated dispersion surfaces in Fig. 2.2 and analytical perturbation theory analysis.

For the zero-energy Dirac point in graphene, there are only the original $\boldsymbol{p}=0$ states in each valley that appear at $\epsilon=0$ upon zone folding. For all three charac- 



$u_{0}=-0.108, u_{1}=0.021, u_{3}=0.021$


$u_{0}=0, u_{1}=0.075, u_{3}=0.075$
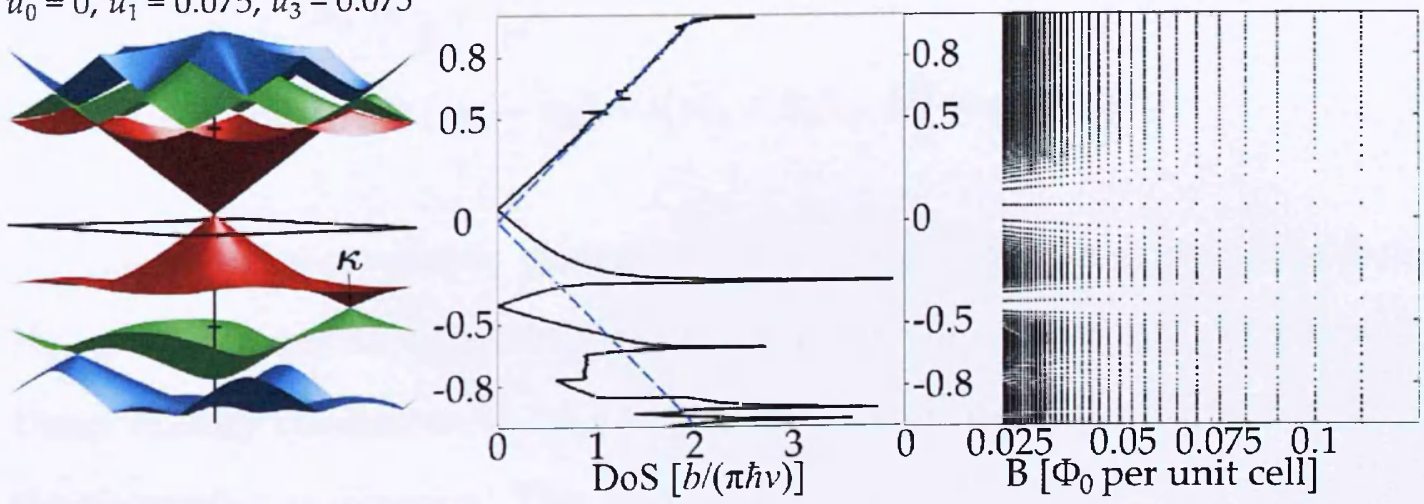

Figure 2.2: Numerically calculated moiré miniband (left), the corresponding density of states (centre), and Landau level spectrum (right) for electrons in the vicinity of graphene's $K$ point. Here we use the rhombic sBZ, so that the $c_{3 v}$ symmetry of the moiré superlattice spectrum is not obviously seen in the images. 
teristic spectra shown in Fig. 2.2, for the inversion-symmetric moiré perturbation, the gapless Dirac spectrum persists at low energies near the conduction-valence band edge with almost unchanged Dirac velocity, $\left[1+O\left(u^{2}\right)\right] v$. The inversionasymmetric terms $\tilde{u}_{i}$ are able [19] to open a minigap at the Dirac point,

$$
\Delta_{0}=24 v b\left|u_{1} \tilde{u}_{0}+u_{0} \tilde{u}_{1}\right|
$$

For the point $\boldsymbol{\mu}=\boldsymbol{b}_{0} / 2$ on the edge of the first sBZ, zone folding brings together two degenerate plane wave states, $|\boldsymbol{\mu}+\boldsymbol{q}\rangle$ and $\left|\boldsymbol{\mu}+\boldsymbol{b}_{3}+\boldsymbol{q}\right\rangle$. The splitting of these degenerate states by the moiré potential in Eq. (2.2) can be studied using degenerate perturbation theory. The corresponding $2 \times 2$ matrix, expanded in small deviation $\boldsymbol{q}$ of the electron momentum from each of the three sBZ $\boldsymbol{\mu}$-points * has the form

$$
\begin{aligned}
& \hat{H}_{\boldsymbol{\mu}+\boldsymbol{q}}=v b\left(\begin{array}{cc}
E_{\mu}+s \frac{q_{y}}{b} & H_{12} \\
H_{12}^{*} & E_{\boldsymbol{\mu}}-s \frac{q_{y}}{b}
\end{array}\right), \\
& E_{\boldsymbol{\mu}} \approx \frac{s}{2}+\frac{s q_{x}^{2}}{b^{2}} \\
& H_{12} \approx\left(s u_{1}-u_{3}\right)-i\left(s \tilde{u}_{1}-\tilde{u}_{3}\right)+2 \frac{q_{x}}{b}\left(u_{0}+i \tilde{u}_{0}\right) .
\end{aligned}
$$

For the inversion-symmetric perturbation, the dispersion relation resulting from Eq. (2.5) contains an anisotropic secondary Dirac point (sDP) [22, 58, 59] with Dirac velocity component $\approx 2 u_{0} v$ in the direction of the sBZ edge and $\approx v$ in the perpendicular direction. This feature is clearly seen at the $\boldsymbol{\mu}$-point of the first moiré miniband in the valence band, in the top row of Fig. 2.2. Note that the electron spectrum is not symmetric between the valence and conduction bands and that the sDPs at the $\boldsymbol{\mu}$-point in the conduction band are obscured by an overlapping spectral branch.

Moving in parameter space, e.g., along the line shown in Fig. 2.1(b), the po-

${ }^{*}$ The Hamiltonian in the vicinity of other two inequivalent points on the sBZ edge, $\boldsymbol{\mu}^{\prime}=$ $\hat{R}_{2 \pi / 3} \boldsymbol{\mu}, \boldsymbol{\mu}^{\prime \prime}=\hat{R}_{4 \pi / 3} \boldsymbol{\mu}$, can be obtained using $H_{\boldsymbol{\mu}+\boldsymbol{q}}=H_{\boldsymbol{\mu}^{\prime}+\hat{R}_{2 \pi / 3} \boldsymbol{q}}=H_{\boldsymbol{\mu}^{\prime \prime}+\hat{R}_{4 \pi / 3} \boldsymbol{q}}$. 
sitions of the three anisotropic sDPs shift along the sBZ edge towards the sBZ corners: either $\boldsymbol{\kappa}=\left(\boldsymbol{b}_{4}+\boldsymbol{b}_{5}\right) / 3$, or $-\boldsymbol{\kappa}$, as shown by arrowed lines in Fig. 2.1(a). In general, a spectrum with three isolated $\mathrm{sDPs}$ at the $\mathrm{sBZ}$ edge is typical for the green volume in the parameter space in Fig. 2.1(b) for the valence band, or Fig. 2.1(c) for the conduction band. In contrast, for $\left(u_{0}, u_{1}, u_{3}\right)$ in the clear part of the parameter space, sDPs on the edge of the first sBZ are overshadowed by an overlapping spectral branch, as is the case on the conduction band side for all three cases shown in Fig. 2.2.

For the points in Fig. 2.1(b,c) on the red and blue surfaces, the three sDPs reach the $\kappa$-point, forming a triple degenerate band crossing, as in the valence band spectrum shown in the middle row of Fig. 2.2, which can be traced using the perturbation theory analysis of the band crossing at $\kappa$ discussed below.

The third line in Fig. 2.2 shows the third type of spectrum of moiré minibands, characteristic for the red and blue volumes of the parameter space in Fig. 2.1. The characteristic feature of such spectra consist of a single isolated sDP, at the $\pm \kappa$-point, in the valence band (Fig. 2.1(b)) or the conduction band (Fig. 2.1(c)).

For the $\kappa$ and $-\kappa$-points, zone folding brings together three degenerate plane wave states, $|\zeta(\boldsymbol{\kappa}+\boldsymbol{q})\rangle,\left|\zeta\left(\boldsymbol{\kappa}+\boldsymbol{b}_{1}+\boldsymbol{q}\right)\right\rangle$, and $\left|\zeta\left(\boldsymbol{\kappa}+\boldsymbol{b}_{2}+\boldsymbol{q}\right)\right\rangle$ (where $\zeta= \pm$ ), whose splitting is determined by

$$
\begin{gathered}
\hat{H}_{\zeta(\boldsymbol{\kappa}+\boldsymbol{q})}=v b\left(\begin{array}{ccc}
\frac{s}{\sqrt{3}}+\frac{s q_{x}}{b} & w_{\zeta} & w_{\zeta}^{*} \\
w_{\zeta}^{*} & \frac{s}{\sqrt{3}}-s \frac{q_{x}-\sqrt{3} q_{y}}{2 b} & -w_{\zeta} \\
w_{\zeta} & -w_{\zeta}^{*} & \frac{s}{\sqrt{3}}-s \frac{q_{x}+\sqrt{3} q_{y}}{2 b}
\end{array}\right), \\
w_{\zeta} \approx \frac{1}{2}\left[\left(u_{0}-2 s \zeta u_{1}+\sqrt{3} \zeta u_{3}\right)+i \zeta\left(\tilde{u}_{0}+2 s \zeta \tilde{u}_{1}-\sqrt{3} \zeta \tilde{u}_{3}\right)\right] .
\end{gathered}
$$

For $w_{\zeta} \neq 0$, the inversion-symmetric terms in $\hat{H}_{\zeta(\kappa+q)}$ partially lift the $\zeta \kappa$-point degeneracy into a singlet with energy $\left(\frac{s}{\sqrt{3}}-2 w_{\zeta}\right) v b$ and a doublet with energies $\left(\frac{s}{\sqrt{3}}+w_{\zeta}\right) v b$, so that a distinctive sDP [59] characterised by Dirac velocity $v_{\kappa}=[1+O(u)] \frac{v}{2}[18]$ is always present at $\pm \kappa$ somewhere in the spectrum. ${ }^{\dagger}$ This

\footnotetext{
${ }^{\dagger}$ Note that the spectra derived from $\hat{H}_{\zeta(\kappa+q)}$ obey the three-fold rotational symmetry.
} 
behaviour reflects the generic properties of the symmetry group of wave vector $\boldsymbol{\kappa}$, detailed in section 2.5, which has the two-dimensional irreducible representation $E$ (corresponding to the sDP) and one-dimensional irreducible representations $A_{1}$ and $A_{2}$. Note that each isolated sDP is surrounded by Van Hove singularities in the density of states corresponding to saddle points in the lowest energy minibands. The weaker inversion-asymmetric terms, $\left|\tilde{u}_{i}\right| \ll\left|u_{i}\right|$, in the second line of Eq. (2.2), open a minigap in both types of sDP discussed above. The perturbation theory leading to Eq. (2.5) and Eq. (2.6) has been performed to greater accuracy, and the sDP positions have been tracked in greater detail, in appendix A.

The appearance of sDPs at the edge of the first miniband results in a peculiar spectrum of electronic Landau levels, as shown on the r.h.s of Fig. 2.2. Each data point in these spectra represents one of the Hofstadter minibands [60] (with an indistinguishably small width) calculated for rational values of magnetic flux, $\frac{p}{q} \Phi_{0}$ per moiré supercell following a method in Ref. [53]. Using these spectra one can trace a clearly separated "zero-energy" Landau level related to the isolated $\kappa$-point sDP in the valence band in the bottom row of Fig. 2.2, in addition to the true zero-energy Landau level at the conduction-valence band edge. The three isolated sDPs on the sBZ edge in the valence band (top row of Fig. 2.2) also result in a "zero-energy" Landau level, though not as clearly separated and split by the magnetic breakdown occurring at $\Phi \approx 0.1 \Phi_{0}$.

\subsection{Effect of the Miniband Spectra on the Hall}

\section{Coefficent}

The inversion-symmetric moiré perturbation will result in either the first sBZ separated from the rest of the spectrum by one or three sDPs, or, for weak perturbations, will result in overlapping first and higher minibands. The experimental consequences of this consists in a non-monotonic variation of the Hall coefficient upon doping the graphene flake with electrons or holes. For example, for those 


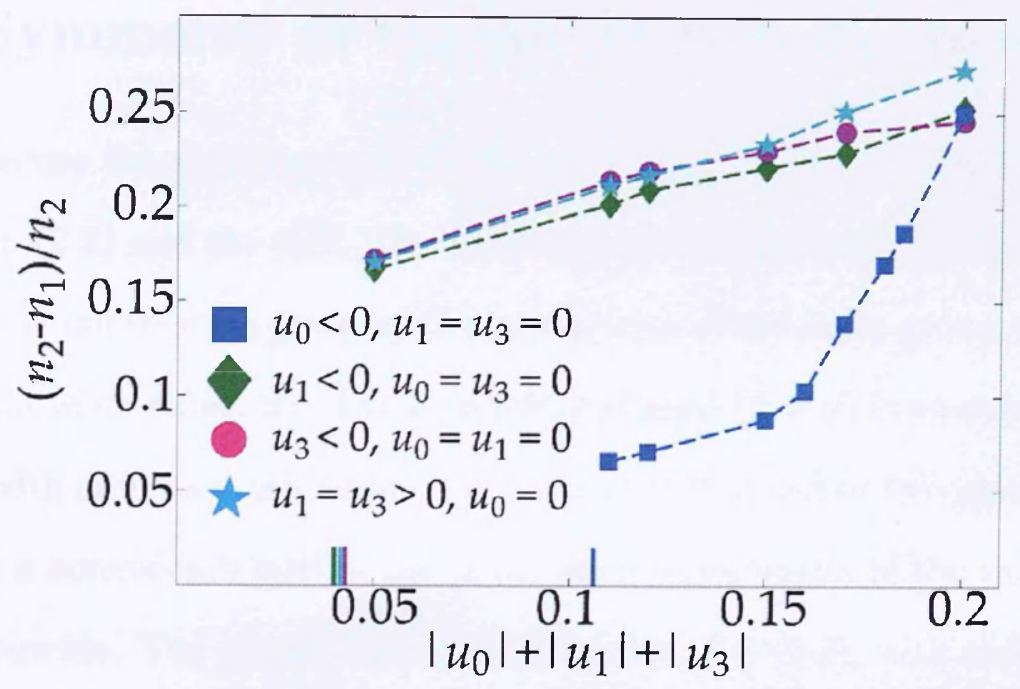

Figure 2.3: The relation between the two densities at which the Hall coefficient in graphene reverses sign upon its doping with holes. The results are shown for several realisations of moiré superlattice in the parameter range corresponding to either three isolated sDPs on the sBZ edge (squares) or one isolated sDP at the $\mathrm{sBZ}$ corner (other symbols). The thresholds for isolation are indicated on the $\mathrm{x}$-axis.

miniband spectra in Fig. 2.2, where there are isolated sDPs in the valence band, the Hall coefficient would pass through a zero value and change sign at two characteristic densities, $n_{1}$ and $n_{2}$. At the density $n_{1}$, which corresponds to the valence band filled with holes up to the Van Hove singularity, the Hall coefficient will change sign from positive to negative. At the higher density, $n_{2}$, which corresponds to a completely filled first miniband, it would repeat the behaviour at the neutrality point changing sign from negative to positive. Such behaviour is expected to take place for the entire regions of the parametric space painted red, blue or green in Fig. 2.1. The relation between these two carrier densities for various types and strengths of moiré perturbations is shown in Fig. 2.3. This behaviour was observed in experimental results discussed in chapter 5 . For the clear part of the parametric space for which we find substantial overlap between many moiré minibands such alternations in the sign of the Hall coefficient would be obscured by the competing contributions from the "electron-like" and "hole-like" branches in the spectrum. 


\subsection{Symmetry of the Moiré Superlattice}

We now discuss the consequences of the moiré superlattice symmetry, both for Hamiltonian (2.2) and the sDP. The point group symmetry of graphene on an incommensurate substrate is given by the intersection of the point group of graphene, $c_{6 v}$, with that of its substrate. For a perfectly aligned $(\theta=0)$ inversion-symmetric substrate, with either a single (dominant) atom per unit cell or two identical atoms arranged in a honeycomb lattice, the point group symmetries of the substrate and graphene coincide. The corresponding Hamiltonian, Eq. (2.2), with moiré harmonics orientated as per Fig. 2.1 (a), must necessarily commute with the operators corresponding to the elements of $c_{6 v}: \hat{c}_{6}, \hat{s}_{x}$ and $\hat{s}_{y}$ which describe $2 \pi / 6$ rotations and reflections that either exchange or preserve the graphene sublattices. The operators for $\hat{c}_{6}$ and $\hat{s}_{y}$ involve the valley exchanging matrices $\tau_{1,2}$ with the result that the symmetry of the Hamiltonian restricted to the $K$ valley, as well as the $K$ valley bandstructure, is reduced to $c_{3 v}=\left\{i d, \hat{c}_{3}, \hat{s}_{x}\right\}$, where $\hat{c}_{3}=\hat{c}_{6}^{2}$ has no intervalley structure. Each of the $\tilde{u}_{i}$ terms are odd under $\hat{c}_{6}$, while the $u_{2}$ and $\tilde{u}_{2}$ terms are odd under $\hat{s}_{y}$, so that these terms are forbidden for the perfectly aligned inversion-symmetric system described above. The point group of substrates with the honeycomb lattice and two non-equivalent atoms per unit cell, such as hBN, only possesses the $\hat{c}_{3}$ and $\hat{s}_{y}$ symmetries which allow inversion-asymmetric parameters $\tilde{u}_{i=0,1,3}$ to take a finite value.

For a finite misalignment angle, the reflection symmetries of graphene and the substrate do not coincide, and the moiré harmonics become misaligned, by an angle $\phi$, from those in Fig. 2.1 (a). However, the moiré harmonics may be brought back into alignment using the transformation $\hat{H}(\boldsymbol{r}) \rightarrow e^{i \sigma_{3} \frac{\phi}{2}} \hat{H}\left(\hat{R}_{\phi} \boldsymbol{r}\right) e^{-i \sigma_{3} \frac{\phi}{2}}$, and the $u_{2}$ and $\tilde{u}_{2}$ terms, which are no longer forbidden, may be gauged away. This procedure restores the reflection symmetries to the Hamiltonian, despite their absence in the geometry of the moiré pattern for finite misalignment angle.

The symmetries described above can be used to gain a deeper understanding of the sDPs discussed in the main text. The $K$ valley plane wave states from the 
three equivalent sBZ corners, $\zeta \kappa_{n=0,1,2}=\zeta \hat{R}_{2 \pi n / 3} \kappa$, which form the basis for $\hat{H}_{\zeta \kappa}$, Eq. (2.6), transform into each other on application of symmetry operators of $c_{3 v}$. In the same basis, the symmetry operators acting on $\hat{H}_{\zeta \kappa}$ take the form of matrices

$$
\Gamma^{\zeta \kappa}\left(\hat{c}_{3}\right)=\left(\begin{array}{ccc}
0 & 0 & -1 \\
-1 & 0 & 0 \\
0 & 1 & 0
\end{array}\right), \quad \Gamma^{\zeta \kappa}\left(\hat{s}_{x}\right)=s \zeta\left(\begin{array}{ccc}
1 & 0 & 0 \\
0 & 0 & 1 \\
0 & 1 & 0
\end{array}\right) .
$$

For the inversion-symmetric superlattice perturbation, the singlet eigenstate of $\hat{H}_{\zeta \kappa}$ is given by $\boldsymbol{v}_{s}=\frac{1}{\sqrt{3}}(1,-1,-1)$. The action of matrices from Eq. (2.7) on this state show that it transforms according to the one-dimensional irreducible representations of $c_{3 v}$ : either $A_{1}$ for $s \zeta=1$ or $A_{2}$ for $s \zeta=-1$, indicating evenness or oddness under $\hat{s}_{x}$ respectively. Similarly, the doublet states of $\hat{H}_{\zeta \kappa}, \boldsymbol{v}_{+}=\frac{1}{\sqrt{3}}\left(\sqrt{2}, \frac{1}{\sqrt{2}}, \frac{1}{\sqrt{2}}\right)$ and $\boldsymbol{v}_{-}=\frac{1}{\sqrt{2}}(0,1,-1)$ transform as the two-dimensional irreducible representation, $E$, and their degeneracy is therefore protected by the $c_{3 v}$ symmetry.

The three anisotropic sDPs can be understood using the compatibility relations in the group appropriate for the sBZ edge, $c_{h}=\left\{i d, \hat{s}_{x}\right\}$. This group only supports one-dimensional irreducible representations $A_{1}$ and $A_{2}$ with the doublet states reducing as $E=A_{1}+A_{2}$. For a given band, $s= \pm 1$, the split bands at $\kappa$ and $-\kappa$ belong to different irreducible representations of $c_{h}$ and therefore cannot be joined along the sBZ edge. Instead, if both of these bands are closer to zero energy than the doublet states, they must each be joined to one of the doublet bands at the opposite sBZ corner. Thus, along the sBZ edge, a crossing of the split bands is required, resulting in the sDPs illustrated in the valence band for the top row of Fig. 2.2. 


\subsection{Microscopic Models}

\subsubsection{Point Charge Model}

The point charge model analysed below mimics the effect of the quadrupole electric moment of the atoms in the top layer of the substrate. In application to the graphene-hBN system, we neglect the potentials of the quadrupole moments of the boron atom, which have only $\sigma$-orbitals occupied by electrons, and replace nitrogen sites by a point core charge $+2|\mathrm{e}|$ compensated by the spread out cloud of the $\pi$-electrons, which we replace by a homogeneous background charge density, giving $-2|e|$ per hexagonal unit cell of the substrate. This model gives an example of an inversion-symmetric moiré superlattice. The matrix elements of the resulting perturbation, taken between sublattice Bloch states $i$ and $j(i, j=A$ or $B)$, acting on the low-energy Dirac spinors of the graphene $K_{+}$valley, are given by the long wavelength components of

$$
\begin{aligned}
\delta H_{i j} & =\frac{-2 \mathrm{e}^{2}}{4 \pi \epsilon_{0}} \sum_{\boldsymbol{R}_{N}} \int d z \frac{L^{2} \Phi_{K_{+} i}^{*}(\boldsymbol{r}, z) \Phi_{K_{+} j}(\boldsymbol{r}, z)}{\sqrt{\left(\boldsymbol{r}-\boldsymbol{R}_{N}\right)^{2}+(z-d)^{2}}} \\
& =\frac{-2 \mathrm{e}^{2}}{4 \pi \epsilon_{0} a / \sqrt{3}} \sum_{\boldsymbol{g}, \boldsymbol{g}^{\prime}, \boldsymbol{g}_{N}} I_{\left|\boldsymbol{K}_{+}+\boldsymbol{g}\right|,\left|\boldsymbol{K}_{+}+\boldsymbol{g}^{\prime}\right|,\left|\boldsymbol{g}_{N}\right|} e^{i\left(\boldsymbol{g}^{\prime}-\boldsymbol{g}+\boldsymbol{g}_{N}\right) \cdot \boldsymbol{r}} e^{i\left(\boldsymbol{g} \cdot \boldsymbol{\tau}_{i}-\boldsymbol{g}^{\prime} \cdot \boldsymbol{\tau}_{j}\right)} .
\end{aligned}
$$

In Eq. (2.8) $\boldsymbol{R}_{N}$ are positions of nitrogen sites and $L^{2}$ is the total area of the graphene sheet; $\Phi_{K_{+}, i}(\boldsymbol{r}, z)$ are Bloch wavefunctions of graphene $\pi$-electrons exactly at the $K_{+}$point. Then the Fourier transform has been used to write $\delta H_{i j}$ in terms of a sum over substrate reciprocal lattice vectors, $\boldsymbol{g}_{N}$, and graphene reciprocal lattice vectors, $\boldsymbol{g}$ and $\boldsymbol{g}^{\prime}$. Nearest neighbour vectors, $\boldsymbol{\tau}_{i=A / B}= \pm(0, a / \sqrt{3})$ are the same as in Fig. 1.1. The homogeneous background charge has not been included in Eq. (2.8) since its only role is to exclude $\boldsymbol{g}_{N}=0$ from the sum. The long wavelength terms in the first exponential of the second line of Eq. (2.8) determine 
$\boldsymbol{b}_{m}=-\left(\boldsymbol{g}^{\prime}-\boldsymbol{g}+\boldsymbol{g}_{N}\right)$. The dimensionless integral,

$$
I_{Q, Q^{\prime}, g_{N}}=\frac{32 a_{0}^{3}}{27(a / \sqrt{3})^{3}} \int d q_{z} d q_{z}^{\prime} \frac{\psi^{*}\left(Q, q_{z}\right) e^{i\left(q_{z}-q_{z}^{\prime}\right) \cdot d} \psi\left(Q^{\prime}, q_{z}^{\prime}\right)}{g_{N}^{2}+\left(q_{z}-q_{z}^{\prime}\right)^{2}}
$$

is written in terms of the Fourier transform of the hydrogen-like graphene $\mathrm{P}^{\mathrm{z}}(\boldsymbol{r}, z)$ orbitals with an effective Bohr radius $a_{0}$,

$$
\begin{aligned}
\psi\left(Q, q_{z}\right) & =\frac{\pi}{a_{0}^{3 / 2}} \frac{1}{2 \pi} \int d \boldsymbol{r} d z e^{-i\left(\boldsymbol{Q} \cdot \boldsymbol{r}+q_{z} z\right)} \mathrm{P}^{\mathrm{z}}(\boldsymbol{r}, z) \\
& =\frac{-64 i a_{0} q_{z}}{\left(1+4 a_{0}^{2}\left(Q^{2}+q_{z}^{2}\right)\right)^{3}}
\end{aligned}
$$

The integral, $I_{Q, Q^{\prime}, g_{N}}$, rapidly decays as a function of the magnitude of all its arguments so that we limit the sum in Eq. (2.8) to only several terms such that $\left|\boldsymbol{K}_{+}+\boldsymbol{g}\right|=\left|\boldsymbol{K}_{+}+\boldsymbol{g}^{\prime}\right|=\left|\boldsymbol{K}_{+}\right|$, with $I=I_{K_{+}, K_{+}, g_{0}}$ where $g_{0}=\frac{4 \pi}{\sqrt{3} a(1+\delta)}$.

The carbon $\mathrm{P}^{\mathrm{z}}$ orbitals may have a different effective Bohr radius compared to hydrogen. The range of values quoted for $\tilde{v}=\frac{2 \mathrm{e}^{2}}{4 \pi \epsilon_{0}(a / \sqrt{3})} I$ in Table 2.1 corresponds to the interval $0.27 \AA \leq a_{0} \leq 0.53 \AA$, indicated by the black double-arrow in Fig. 2.4, where the interlayer separation $3.22 \AA \leq d \leq 3.5 \AA$ is taken from Ref. [61]. The resulting superlattice perturbation and dispersion surfaces are shown graphically in Fig. 2.5 .

Both the dominance of the simplest moiré harmonics, and the finite values for the off-diagonal terms $u_{1}$ and $u_{2}$, stem from the three-dimensional treatment of the substrate potential. The potential is strongest near the substrate, and therefore a greater proportion of the integral $I_{Q, Q^{\prime}, g_{N}}$ comes from the region near the substrate, where the graphene $\mathrm{P}^{\mathrm{z}}$ orbitals are broad and therefore have both rapidly decaying Fourier components and significant overlap with their neighbours. This contrasts with the model employed in Ref. [18] which is based on a two-dimensional substrate potential resulting in $u_{1}=u_{2}=0$. 


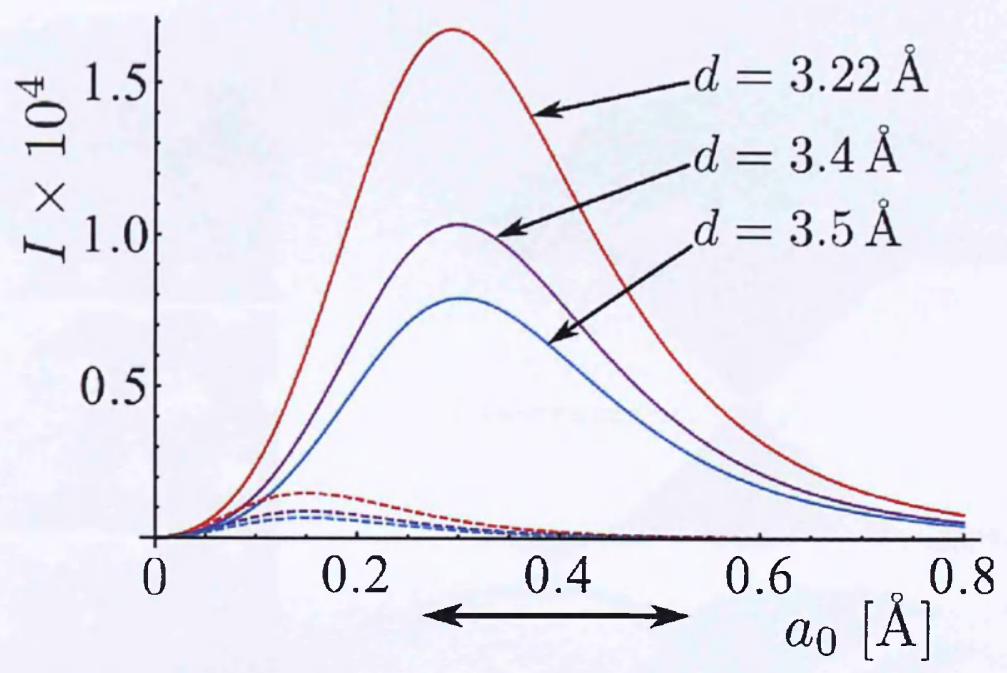

Figure 2.4: Solid lines show the dimensionless integral $I$, as a function of the effective Bohr radius of the graphene $\mathrm{P}^{\mathrm{z}}$ orbitals, for various choices of interlayer separation $d$. To demonstrate convergence of the sum in Eq. (2.8), dashed lines show $I_{2 K_{+}, K_{+}, g_{0}}$ for the same values of $d$.

\subsubsection{Graphene-hBN Hopping Model}

In Ref. [19], Kindermann et al. modelled a hBN substrate as a lattice of $\mathrm{P}^{\mathrm{z}}$ orbitals onto which the graphene electrons can hop. This treatment, extended from a model of twisted bilayer graphene [62], assumed equal values for the hopping integral to the boron and nitrogen sites, with the difference between the two sublattices arising from their different on-site energies. Here we consider an inversion-symmetric version of the hopping model of Ref. [19], assuming that coupling between graphene and the hBN layer is dominated by the hopping to only one of the two sublattices (e.g. boron). Using $\boldsymbol{k} \cdot \boldsymbol{p}$ theory (section 1.4 ), this coupling can be written in the basis of graphene $K_{+}$valley Bloch states, $\left(\Phi_{K_{+}, A}, \Phi_{K_{+}, B}\right)$, as [19],

$$
\begin{aligned}
& \delta \hat{H}=\hat{H}_{\mathrm{int}} \frac{1}{\epsilon-V-m} \hat{H}_{\mathrm{int}}^{\dagger}, \\
& \hat{H}_{\text {int }}=\frac{\gamma}{3} \sum_{n=0,1,2} e^{-i\left(\hat{R}_{\frac{2 \pi n}{3}} \kappa\right) \cdot r}\left(\begin{array}{c}
e^{i \frac{2 \pi n}{3}} \\
e^{-i \frac{2 \pi n}{3}}
\end{array}\right) .
\end{aligned}
$$

Neglecting a non-oscillatory term, which corresponds to a trivial constant energy shift, Eq. (2.9) as applied to graphene electrons in valley $K_{+}$, leads to the moiré 
a)

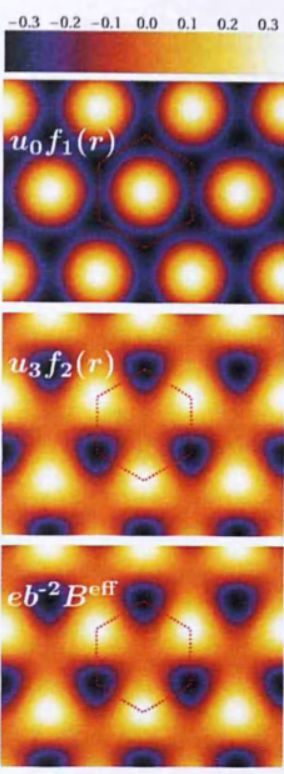

b)

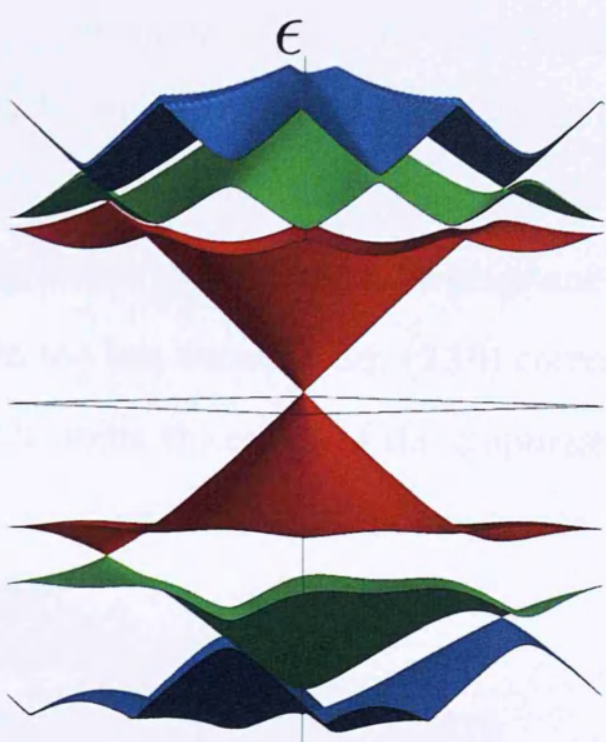

Figure 2.5: a) The simple potential modulation, $u_{0} f_{1}(\boldsymbol{r})$, the local sublattice asymmetry, $u_{3} f_{2}(\boldsymbol{r})$, and pseudo-magnetic field, $e b^{-2} B^{\text {eff }}=u_{1} f_{2}(\boldsymbol{r})$ for the choice of parameters corresponding to the point charge model or the hopping model scaled so that $\left|u_{0}\right|+\left|u_{1}\right|+\left|u_{3}\right|=0.15$. b) The corresponding miniband spectra.

Hamiltonian, Eq. (2.2), with

$$
\left\{u_{i=0, \ldots 3}\right\}=\frac{\gamma^{2} /(v b)}{9(m+V)}\left\{\frac{1}{2}, \frac{-\delta}{\sqrt{\delta^{2}+\theta^{2}}}, \frac{\theta}{\sqrt{\delta^{2}+\theta^{2}}},-\frac{\sqrt{3}}{2}\right\} .
$$

The parameters of the superlattice perturbation given in Table 2.1 of the main text, correspond to $\gamma=0.3 \mathrm{eV}, V=0.8 \mathrm{eV}$ and $m=2.3 \mathrm{eV}$, in accordance with Ref. [19]. For the perfectly aligned system, we always find $u_{2}=0$, which is a consequence of the reflection symmetries present in the perfectly aligned substrate-graphene system (see section 2.5).

\subsubsection{Inversion Asymmetric Microscopic Models}

To generate the inversion-asymmetric perturbation terms in the microscopic models discussed above, we now account for both the nitrogen and the boron sublattice of the hBN underlay. This is achieved by taking the moiré perturbation,

$$
\delta \hat{H}^{\prime}(\boldsymbol{r})=\delta \hat{H}(\boldsymbol{r})+v_{B} \delta \hat{H}\left(\boldsymbol{r}-\frac{4 \pi}{3 b^{2}} \boldsymbol{b}_{0}\right) .
$$


Here $\delta \hat{H}(\boldsymbol{r})$ is the Hamiltonian $(2.8)$, and $v_{B} \ll 1$ controls the strength of the perturbation due to the boron sites with respect to that of the nitrogen sites. The choice of origin used for $\delta \hat{H}^{\prime}$ in Eq. (2.10) corresponds to the location in the moiré unit cell with the nitrogen site directly below the centre of the graphene hexagon; whereas the coordinate shift $-\frac{4 \pi}{3 b^{2}} \boldsymbol{b}_{0}$ in the last terms of Eq. (2.10) corresponds to the position with the boron site directly under the centre of the graphene hexagon. This coordinate change in the last term affects a rotation in the parameter space $\ddagger$ for $v_{B} H\left(\boldsymbol{r}-\frac{4 \pi}{3 b^{2}} \boldsymbol{b}_{0}\right)$

$$
\left(\begin{array}{c}
v_{B} u_{i} \\
v_{B} \tilde{u}_{i}
\end{array}\right) \rightarrow \hat{R}_{\alpha 2 \pi / 3}\left(\begin{array}{c}
v_{B} u_{i} \\
v_{B} \tilde{u}_{i}
\end{array}\right), \quad \alpha=\left\{\begin{array}{cc}
-1 & \text { for } i=0 \\
1 & \text { otherwise }
\end{array} .\right.
$$

Hence the parameters are given by

$$
\begin{aligned}
& \left\{u_{i=0, \ldots 3}\right\}=v^{+}\left\{\frac{1}{2}, \frac{-\delta}{\sqrt{\delta^{2}+\theta^{2}}}, \frac{\theta}{\sqrt{\delta^{2}+\theta^{2}}},-\frac{\sqrt{3}}{2}\right\}, \\
& \left\{\tilde{u}_{i=0, \ldots 3}\right\}=v^{-}\left\{\frac{-1}{2}, \frac{-\delta}{\sqrt{\delta^{2}+\theta^{2}}}, \frac{\theta}{\sqrt{\delta^{2}+\theta^{2}}},-\frac{\sqrt{3}}{2}\right\},
\end{aligned}
$$

where $v^{+}=\tilde{v}\left(1-\frac{v_{B}}{2}\right)$ and $v^{-}=\tilde{v} \frac{\sqrt{3} v_{B}}{2}$. It is interesting to note that, for this choice of parameters, the zero-energy Dirac point remains un-gapped, due to cancellation of the two terms in Eq. (2.4) that is peculiar to this choice of parameters. However, gaps in the sDPs are opened as expected.

\subsection{Conclusion}

Using a general symmetry-based approach, we have provide a classification of generic miniband structures for electrons in graphene placed on substrates with the hexagonal Bravais symmetry. In particular, we identify conditions at which the first moiré miniband is separated from the rest of the spectrum by either one,

\footnotetext{
${ }^{\ddagger}$ In Eqs. (2.5) and (2.6) this transformation is equivalent to a gauge transform and therefore leaves the bandstructure unaltered.
} 
or a group of three isolated mini Dirac points, and is not obscured by dispersion surfaces coming from other minibands. In such cases the Hall coefficient exhibits two distinct alternations of its sign as a function of charge carrier density. Other experimental consequences of the miniband spectra will be discussed in the next three chapters. 


\section{Chapter 3}

\section{Optical Absorption in}

\section{Graphene-hBN Heterostructures}

\subsection{Introduction}

In this chaper, we investigate how the characteristic features of the moiré minibands of graphene-hBN heterostructures, described in chapter 2 , are reflected in the absorption spectra in the infrared to visible optical range. It has been noticed in recent tight-binding model studies $[63,64]$ that the absorption of light by Dirac electrons in twisted Bilayer graphene (which also feature a moiré superlattice) acquires robust features due to the edges and van Hove singularities of the first minibands. These are affected by both the modulation of the density of states and the sublattice structure of the electron Bloch states in graphene modified by the superlattice. The purpose of this chapter is to establish what characteristic features in the graphene-hBN heterostructure absorption spectrum can be attributed to one or another combination of moiré parameters in the phenomenological Hamiltonian Eq. (2.2), with a view to narrowing down their choice based on the combination of the transport data with the forthcoming optical studies.

The inversion-symmetric perturbation in (2.2) determines a gapless miniband spectrum, with the sDP singularities either at the edge of the first miniband, or 
embedded into a continuous spectrum at higher energies, whereas the asymmetric part opens a 'zero-energy' gap $\Delta_{0}$ and gaps $\Delta_{1}$ at the secondary Dirac points in conduction and valence bands ( $s= \pm 1$ respectively),

$$
\begin{array}{r}
\Delta_{0}=24 v b\left|u_{1} \tilde{u}_{0}+u_{0} \tilde{u}_{1}\right|, \\
\Delta_{1}=\sqrt{3}\left|\tilde{u}_{0}+2 s \zeta \tilde{u}_{1}-\sqrt{3} \zeta \tilde{u}_{3}\right| .
\end{array}
$$

However, recent transport experiments [23, 25] did not show any pronounced gap at the miniband edges, and either no gap [23] (see discussion in section 5) or a small gap [25] at zero energy $(\Delta \sim 20 \mathrm{meV})$, telling us that the inversion-asymmetric part of the moiré superlattice potential is weak. This agrees with the ansatz made in in section 2.6 that only one out of the two sublattices (either $\mathrm{N}$ or B atoms) of the honeycomb lattice of $\mathrm{hBN}$ top layer dominates in the coupling with the graphene electrons, thus making the effective lattice of the $\mathrm{hBN}$ perturbation simple hexagonal and prescribing inversion symmetry to the moiré potential. Therefore, in the following we assume that $\left|\tilde{u}_{i}\right| \ll\left|u_{i}\right|$ and neglect the inversion asymmetric terms in the analysis of optical absorption in the infrared-to-visible range.

Besides the above described dominance of the inversion-symmetric part in the moiré superlattice potential, very little is known for definite about the values of the superlattice parameters in Eq. (2.2). Two microscopic models, based on either scattering of graphene electrons by the quadrupole electric moments of nitrogen (section 2.6.1), or on hopping between graphene carbons and hBN atoms (section 2.6.2), predict similar relationships between coupling constants $u_{0}, u_{1}$, and $u_{3}$ in $(2.2)$,

$$
u_{0}=\frac{\tilde{v}}{2 v b}, \quad u_{1}=\frac{-\tilde{v} \delta}{v b \sqrt{\delta^{2}+\theta^{2}}}, \quad u_{3}=-\frac{\sqrt{3} \tilde{v}}{2 v b}
$$

with $0.6 \mathrm{meV} \leq \tilde{v} \leq 3.4 \mathrm{meV}$, to compare with $v b \approx 340 \mathrm{meV}$ for $\theta=0$ and $v b \approx 750 \mathrm{meV}$ for $\theta=2^{\circ}$. However, rather simplistic approximations are used in these models, and one must assume much larger values of superlattice potential parameters to relate the theory in chapter 2 to the recent magneto-transport data. 
This suggests that these models show that all three inversion-symmetric interaction terms in the Hamiltonian in (2.2) should be taken into account in a comprehensive phenomenological theory of moiré superlattice in graphene-hBN heterostructures. Note that the strongest deviations of the optical absorption by electrons in a heterostructure from the universal graphene absorption coefficient $g_{1}=\pi e^{2} / \hbar c$ would be most pronounced in a spectral range around $\omega \sim v b$ (from infrared at $\theta=0^{\circ}$ to visible at $\theta \sim 5^{\circ}$ ): for much lower photon frequencies, electron states are almost the same as in the unperturbed Dirac spectrum, whereas photons of much higher energies involve transitions between numerous overlapping minibands such that individual spectral features would be smeared out by the faster inelastic relaxation of photoexcited electrons and holes.

The coefficient of absorption of light described by energy $\omega$ and polarization $\mathbf{e}$ is

$$
g(\omega)=\frac{8 \pi \hbar}{c \omega \mathcal{A}} \operatorname{Im} \sum_{\mathbf{p}, s, s^{\prime}} \frac{f_{\mathbf{p} s^{\prime}}-f_{\mathbf{p} s}}{\omega+\epsilon_{\mathbf{p} s}-\epsilon_{\mathbf{p} s^{\prime}}+i \eta} M_{\alpha \beta}^{s s^{\prime}} e_{\alpha}^{*} e_{\beta}
$$

where $\alpha, \beta=x, y, \epsilon_{\mathrm{p} s}$ stands for the miniband energy found by diagonalisation of the Hamiltonian in (2.2), $f_{\mathrm{p} s}$ are the occupation numbers, $\mathcal{A}$ is the normalization area of the miniband plane wave states, and $\eta$ is the broadening of the energy states (we take $\eta=v b / 200$ unless otherwise stated). We also find numerically the eigenstates of $H$ to calculate the matrix elements of the current operator,

$$
M_{\alpha \beta}^{s s^{\prime}}=\left\langle\mathbf{p} s\left|\hat{j}_{\alpha}^{\dagger}\right| \mathbf{p} s^{\prime}\right\rangle\left\langle\mathbf{p} s^{\prime}\left|\hat{j}_{\beta}\right| \mathbf{p} s\right\rangle
$$

where $j_{\alpha}=e v \sigma_{\alpha}$ are Dirac current operators. The above equation gives the selection rules for optical transitions between the miniband states (we neglect the momentum transfer due to absorption of the photon), and take into account the spin and valley degeneracy. The $c_{3}$ rotational symmetry of the moiré pattern implies that there is no dependence of $g(\omega)$ on the polarization angle of the light, and after taking into account the fact that the two valleys in the graphene spectrum are related by time-inversion symmetry, we conclude that the absorption spectrum 
(a)
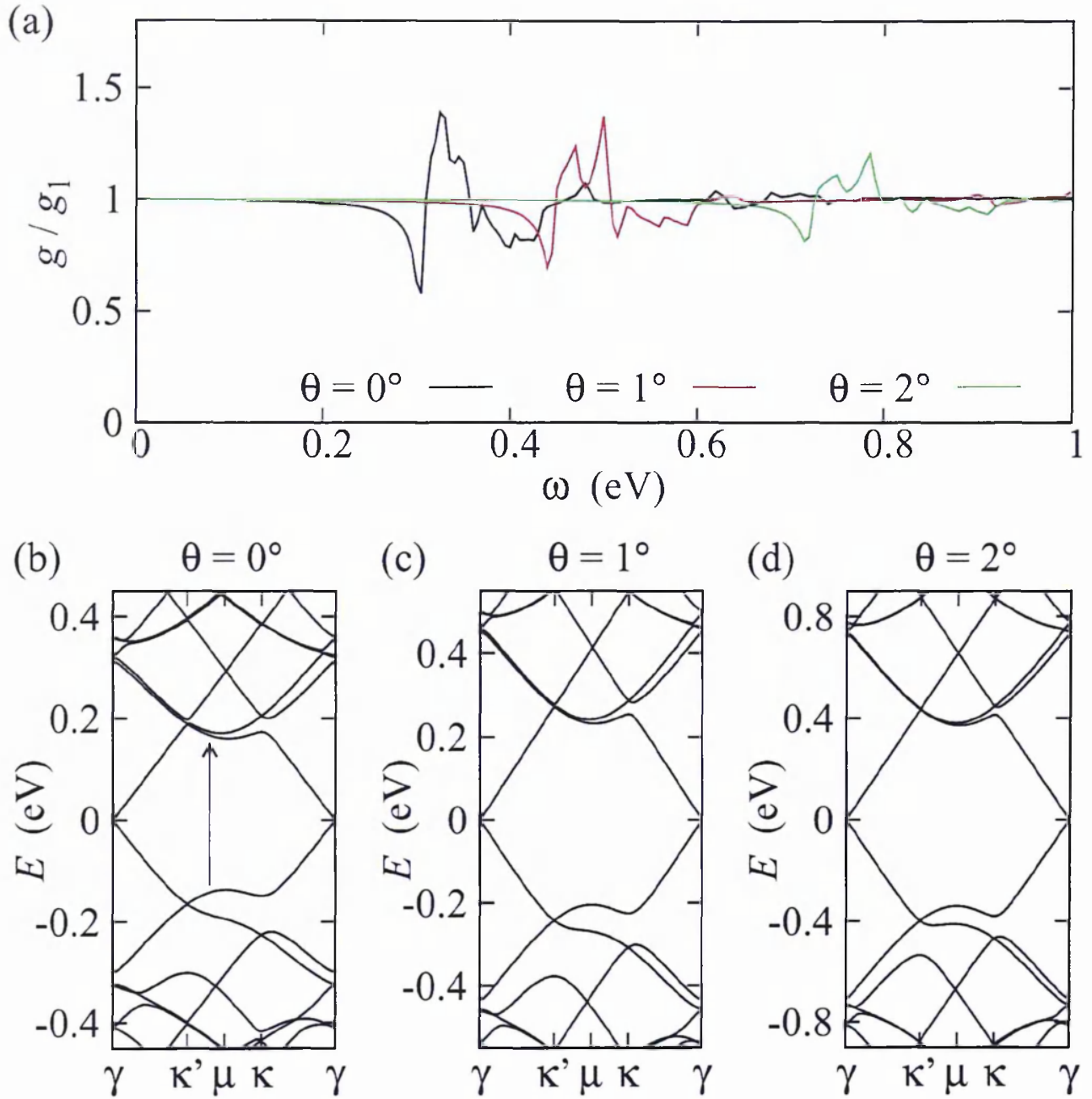

Figure 3.1: (a) The optical absorption spectra for the model moire perturbation in with parameters in (3.1) for $\tilde{v}=17 \mathrm{meV}$ and various misalignment angles. The Fermi energy, $\epsilon_{F}=0$. (b)-(d) Band structures corresponding to each of the spectra in (a). We have marked transitions responsible for the absorption maxima in (b).

is independent of the polarization state of photons.

\subsection{Optical Absorption Spectra}

We discuss the features of the absorption spectrum specific to the various realisations of the moire superlattice perturbation. In Fig. 3.1(a) we show the features of the absorption spectrum calculated for the realization of the moire superlattice with substantially sizeable amplitudes and the weight of parameters $u_{0}, u_{1}$, and $u_{3}$ set in (3.1). In this case, the electron spectrum features a sDP at the edge of the first miniband on the valence band side and overlapping bands on the conduction 
band side. It is strongly electron-hole asymmetric, which makes the spectral features of the superlattice less pronounced. The optically active transitions which provide the deviation from the standard absorption $g(\omega)=g_{1}$ come from the edge of the mBZ, as shown by the arrow in Fig. 3.1(b). This figure also shows the tendency of the spectral features to stretch into higher energies and gradually decrease in size with increasing misalignment angle.

It is also instructive to analyse spectra for more peculiar realizations of moiré superlattice, starting only with one of the three terms in the perturbation, and then increasing the size of the others. The corresponding evolution of the absorption spectra is shown in Fig. 3.2(a) for each of the three interaction terms. The distinctive feature of a pure $u_{0}$ interaction [black line, band structure shown in Fig. 3.2(b)] is the double peak structure near $\omega / v b \approx 0.8$. Fig. 3.2(e) shows the spectra for the same $u_{0}$ interaction with a small $u_{1}$ or $u_{3}$ interaction added for the frequency interval near the double peak. For both added interactions, each part of the double peak is split in two destroying this simple structure. Therefore, the most obvious identifying feature of a strong $u_{0}$ interaction is masked by even weak additions of the other two interactions. The red line in Fig. 3.2(a) is the spectrum for the $u_{1}=0.15$ interaction, with the associated band structure in Fig. 3.2(c). There are two key features to this spectrum, the first being that the initial deviation from the standard $g(\omega)=g_{1}$ result for low $\omega$ is downwards, not upwards as for the pure $u_{0}$ and $u_{3}$ interactions. The second key feature is the strong single peak at $\omega / v b \approx 0.8$ due to the electron-hole symmetry of the $u_{1}$ interaction allowing van Hove singularities in both the valence and conduction bands to contribute to the absorption simultaneously, as indicated by the double arrow marked ' $(1)$ ' in Fig. 3.2(c). In Fig. 3.2(f) we show this peak with a strong mixture of the $u_{0}$ and $u_{3}$ interactions [compare the size of the perturbation to that in Fig. 3.2(e)]. For both additional interactions, the position of the peak has shifted a little, and the peak has decreased slightly in height, but the peak is still clearly identifiable indicating that this spectral feature is rather robust against perturbation by the other 
two interactions. Finally, the $u_{3}=0.15$ interaction is shown by the green line in Fig. 3.2(a) and the band structure in Fig. 3.2(d). The identifying feature in this case is the small peak followed by a large frequency range where the absorption is suppressed substantially below the value of $g_{1}$.

Fig. 3.3 illustrates several examples of how the absorption spectrum would be modified by change in the carrier density (and Fermi energy $\epsilon_{F}$ ) in the heterostructure. In contrast to unperturbed graphene (where Pauli blocking simply suppresses absorption at $\omega<2 \epsilon_{F}$ ) here, due to Bragg scattering of electrons by the superlattice potential, empty states in higher minibands of the valence band or filled states in higher minibands of the conduction band open new absorption channels.

\subsection{Conclusion}

In conclusion, we have demonstrated that optical spectroscopy with infra-red and visible radiation may be used to gain insight into the detailed characterization of the interaction between layers in graphene-hBN heterostructures with a small misalignment angle. Since the exact parameters of this interaction are unknown, we have described the general features of optical spectroscopy due to each of the interaction terms allowed by symmetry, and linked these parameters to the formation of secondary Dirac points in the heterostructure spectrum and nearby van Hove singularities in the moiré miniband spectra. We also show that the modification of the optical transitions rules, due to the Bragg scattering of graphene electrons off the moiré superlattice, modify the doping dependence of graphene absorption spectrum, in a manner very sensitive to the detailed structure of moiré superlattice potential. 

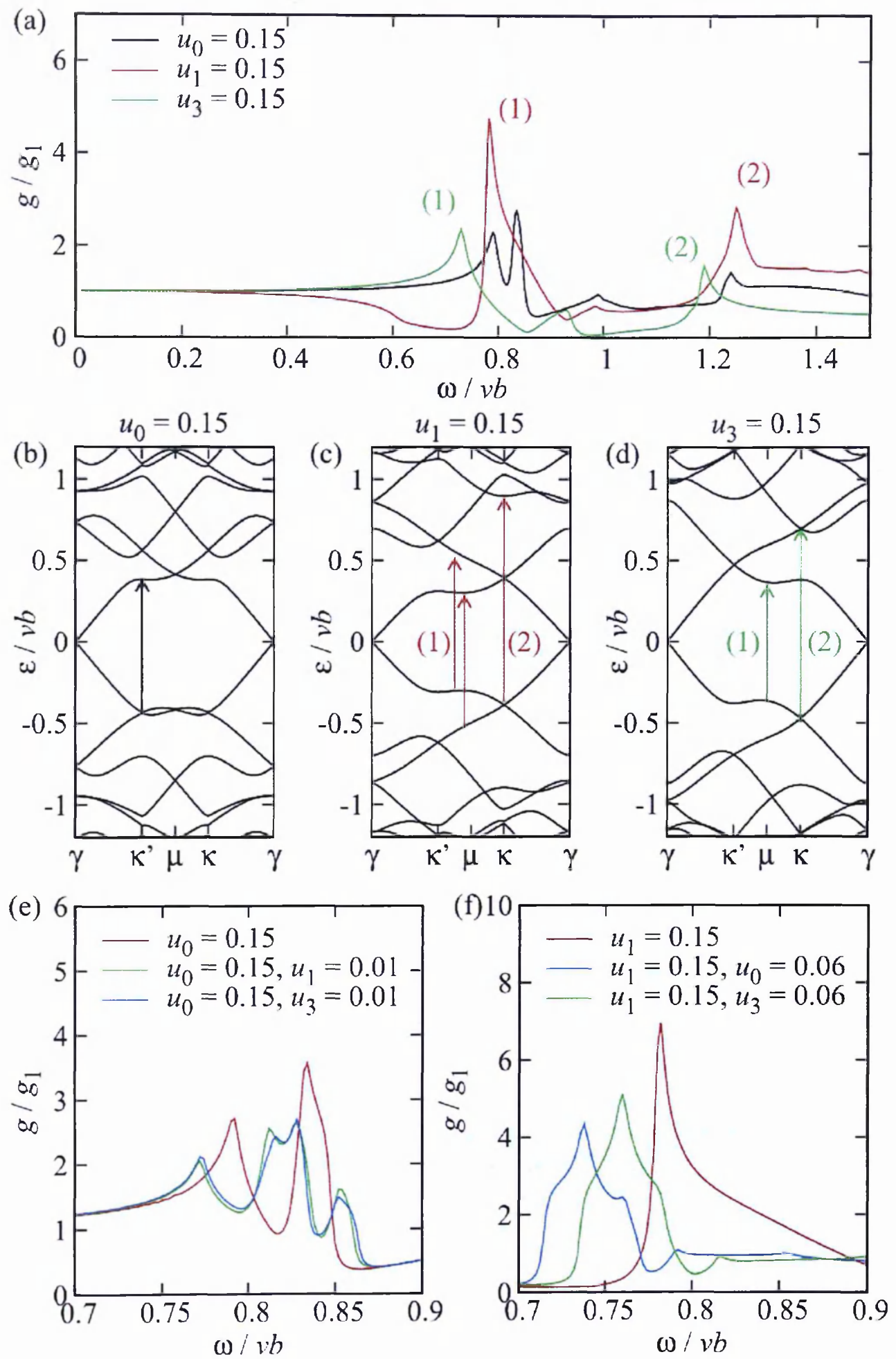

Figure 3.2: (a) Absorption spectra for each of the interaction terms in (2.2), and Fermi energy, $\epsilon_{F}=0$.. (b)-(d) The corresponding band structures with the transitions that make the strongest contribution to the labelled peaks in (a) marked with vertical arrows. (e) The change in the $u_{0}=0.15, u_{1}=u_{3}=0$ double peak at $\omega / v b \approx 0.8$ with addition of weak $u_{1}$ and $u_{3}$ interaction terms. (f) The change in the $u_{1}=0.15, u_{0}=u_{3}=0$ peak at $\omega / v b \approx 0.8$ due to the addition of strong electron-hole symmetry-breaking terms $u_{0}$ and $u_{3}$. In (e) and (f), we have $\eta=v b / 500$. 

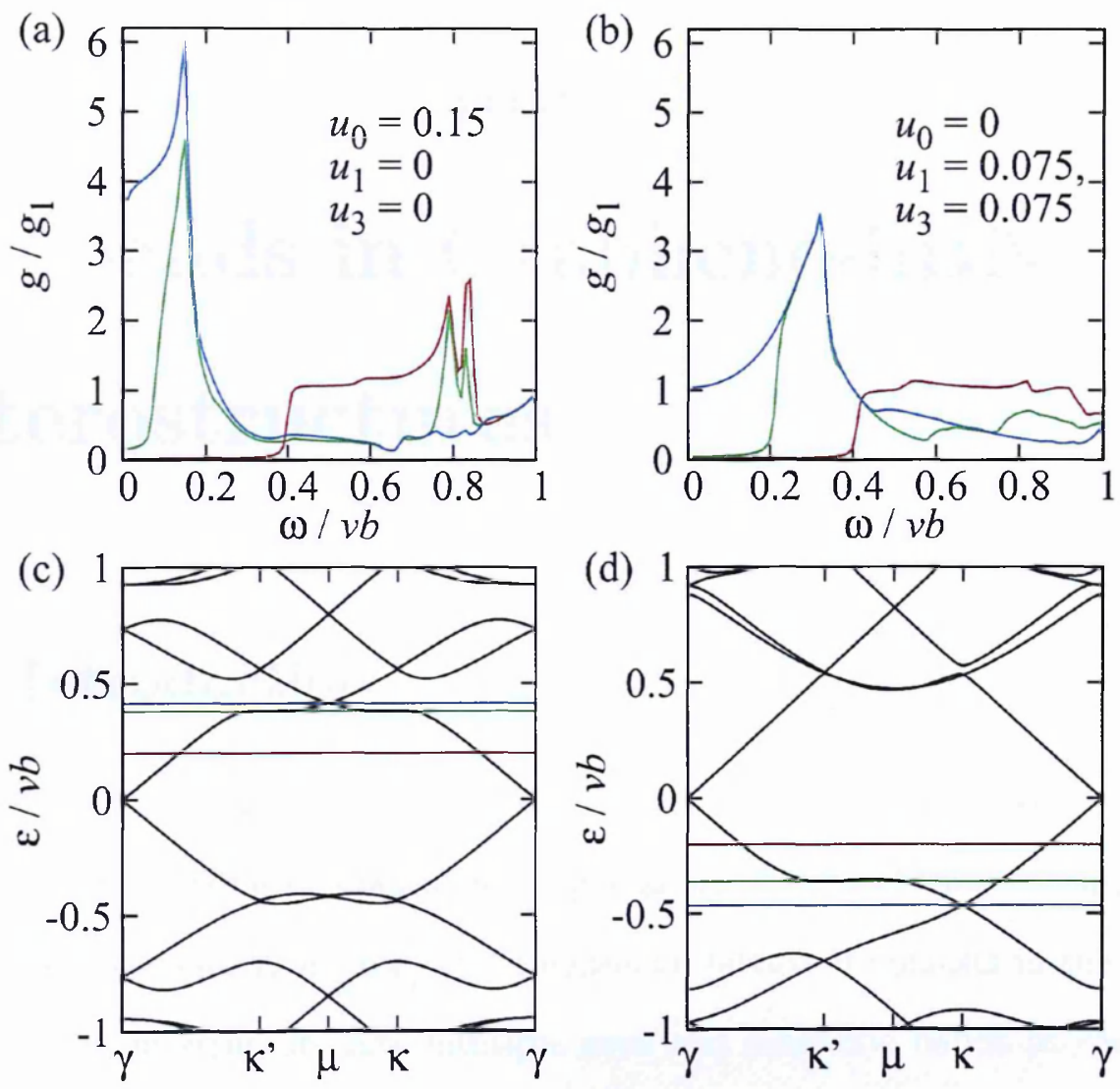

Figure 3.3: Variation of the optical spectrum with $\epsilon_{F}$. (a) Optical absorption spectra for the conduction band containing three sDPs on the $\mathrm{mBZ}$ edge. (b) Optical absorption spectra for the valence band containing one sDP at the mBZ corner. (c) and (d) Band structures associated with (a) and (b), respectively. The horizontal coloured lines show the Fermi energy for each of the spectra in (a) and (b). 


\section{Chapter 4}

\section{Fractal Spectrum of Magnetic}

\section{Minibands in Graphene-hBN}

\section{Heterostructures}

\subsection{Introduction}

The fractal spectrum of electron magnetic bands in crystals subjected to a strong magnetic field $[65,66]$ is one of the most spectacular results of the quantum theory of solids [67]. For electrons on a two-dimensional lattice, it consists in the fracturing of their band structure into multiple gaps and magnetic bands at each value $B_{\frac{p}{q}}=\frac{p}{q} \phi_{0} / S$ of the field providing a rational fraction of magnetic flux quantum, $\phi_{0}$, per unit cell area, $S$, of the crystal, [65, 67]. Its graphical representation [60], obtained for a square lattice model with the nearest neighbour hopping, known as the Hofstadter butterfly, offers an attractive image of a hierarchical self-similarity in physics, which has stimulated numerous experimental attempts to observe the fractal spectrum of electrons in quantum transport measurements. Since the sparsity of the spectrum increases and the size of the gaps decrease for larger values of the denominator $q$ of the rational fraction, the observation of fractal magnetic band spectra in real crystals would require unsustainably strong magnetic fields. Hence, 
the early efforts in observing the Hofstadter butterfly spectrum of electrons were based on two-dimensional electron gases in periodically patterned GaS/AlGaAS heterostructures [68], where the superimposed superlattice period was made large enough to obtain the low denominator fractions within the experimentally available steady magnetic field range $B \leq 35 \mathrm{~T}$. The more recent observation of hexagonal moiré superlattices in graphene, residing on substrates with hexagonal lattice facets [48], has shown an alternative way towards creating a longrange periodic potential for electrons: by making lattice-aligned graphene heterostructures with a hexagonal crystal with an almost commensurate period. For this, hexagonal boron nitride provides a perfect match, and, during the last year, several observations of moiré superlattice effects in graphene-hBN heterostructures have been reported [23-25].

In this chapter, we use the phenomenological model described in chapter 2 , to study generic features of Zak's magnetic minibands of electrons, in graphenehBN heterostructures. Graphene's specific electronic properties play an important role in this study. First of all, due to the long-range of the superlattice period, we describe graphene electrons using the effective Dirac theory, where the form of moiré perturbation depends on the detailed structure and symmetry of the underlying surface. In particular, for an inversion-symmetric surface layer, the zero-magnetic-field spectra features isolated secondary Dirac points at the first miniband edge. Here we find that generations of Dirac electrons systematically reappear at the edges of Zak's magnetic minibands for values $\frac{p}{q} \phi_{0}$ of the magnetic flux through a moiré supercell, and that fractal Hofstadter spectra in the vicinity of such points can be described in terms of $4 q$-times degenerate Landau levels of gapped Dirac electrons in an effective magnetic field $\delta B=B-B_{\frac{p}{q}}$. As the Landau level spectrum of Dirac electrons contains a zero-energy level, separated by the largest gap from the rest of the spectrum, this determines a specific hierarchy of minigaps in the Hofstadter butterfly, resulting in a peculiar sequence of dominant incompressible states of electrons in graphene-hBN heterostructures in a strong 


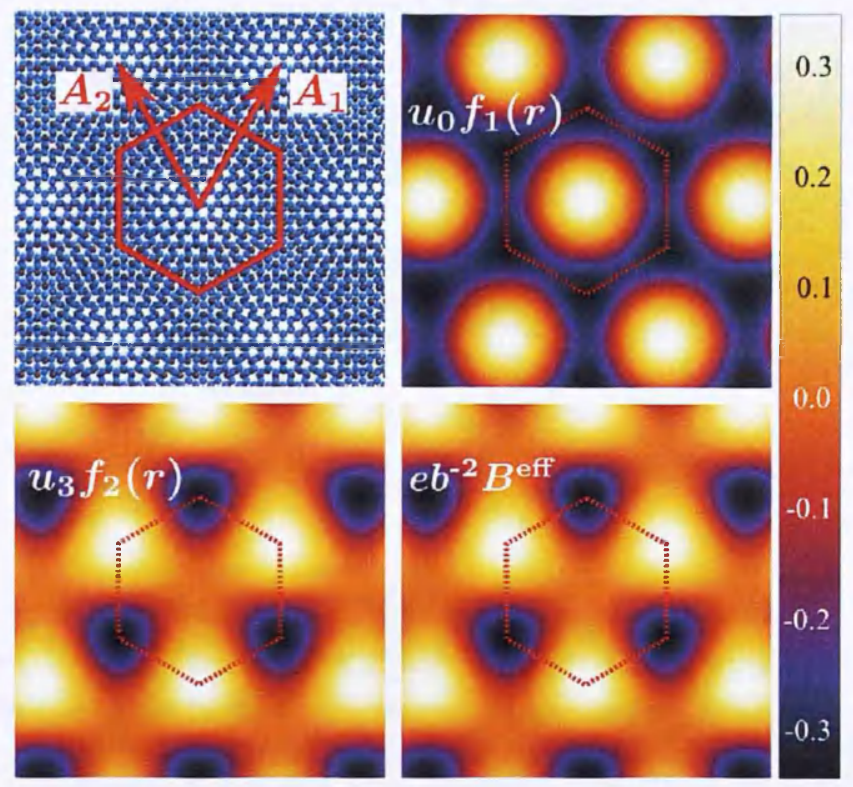

Figure 4.1: The moiré pattern of graphene on a hBN underlay, shown with an exaggerated lattice mismatch (top left), and the moiré perturbation (remaining panels) with $v^{-}=\theta=0$ and $v^{+}=0.063 v b$. The pseudo magnetic field, associated with $u_{1}$ is shown in the lower right panel. The red hexagon shows the moiré unit with area $S=\frac{\sqrt{3}}{2} A^{2}$, and the lattice vectors $\boldsymbol{A}_{1}=A(1 / 2, \sqrt{3} / 2), \boldsymbol{A}_{2}=A(-1 / 2, \sqrt{3} / 2)$.

magnetic field.

\subsection{Magnetic Translational Symmetry and Cal-}

\section{culation of Zak's Magnetic Minibands}

In the presence of a strong magnetic field, the electron spectrum can be described in terms of Zak's magnetic minibands for any value of $B$, providing a rational value of magnetic flux through the unit cell of the moire pattern,

$$
\phi \equiv B S=\frac{p}{q} \phi_{0}
$$

Here $p$ and $q$ are coprime and $\phi_{0}=h / e$. This ensures a commensurability between the moire superlattice and the superlattice with one flux quantum per super cell. One usually $[65,67,69,70]$ exploits the commensurability in Eq. (4.1) by enlarging the unit cell $q$-times in, e.g., the $\boldsymbol{A}_{1}$ direction, which would imply a magnetic 


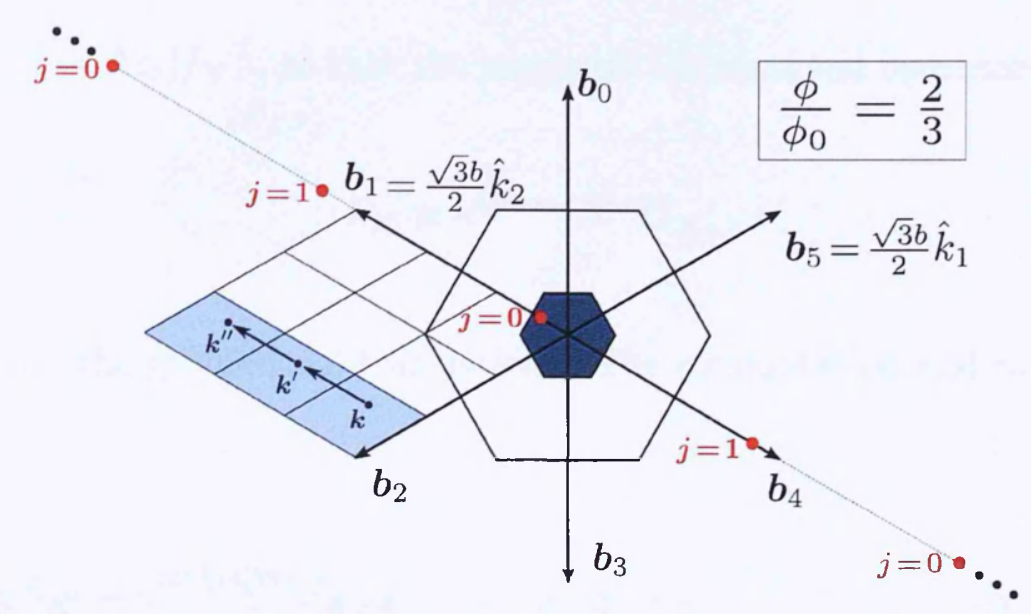

Figure 4.2: The moiré Brillouin zone (large hexagon, with area $\sqrt{3} b^{2} / 2$ ), the moiré reciprocal lattice vectors $\boldsymbol{b}_{m}$. The $q$-times smaller and $q^{2}$-times smaller magnetic Brillouin zones (described in the main text) for $\phi / \phi_{0}=2 / 3$ are shown in light blue and dark blue respectively. The chain of $k_{2}$ values (red dots) used to construct Bloch states (4.2) are also shown.

Brillouin Zone $q$-times smaller than the moire Brillouin zone in the $\boldsymbol{b}_{2}$ direction, as shown in Fig. 4.2, with the dispersion repeated inside it with the period $\boldsymbol{b}_{1} / q$. However, to preserve the point group symmetries, here we enlarge the unit cell $q$-times in both the $\boldsymbol{A}_{1}$ and $\boldsymbol{A}_{2}$ directions. With this choice the magnetic Brillouin zone (Fig. 4.2) is $q^{2}$-times smaller than the moiré Brillouin zone, and zone folding of the periodically repeating dispersion onto the smaller Brillouin zone provides a $q$-fold degeneracy of all energy states in its bands [66].

The classification of Zak's magnetic minibands, and their degeneracies, can be deduced from the properties of the group, $\left\{S_{\boldsymbol{X}}: \boldsymbol{X}=m_{1} \boldsymbol{A}_{1}+m_{2} \boldsymbol{A}_{2}\right\}$, of magnetic translations. Here we follow section 1.7, and use a non-orthogonal coordinate system, adapted to the hexagonal symmetry of the moiré pattern,

$$
r=x_{1} \hat{x}_{1}+x_{2} \hat{x}_{2}
$$

with basis vector $\hat{x}_{i=1,2}=A_{i} / A$ directed along the the lattice vectors of the moiré pattern (see Fig. 4.1). Also, we use the Landau gauge for the vector potential, 
$\boldsymbol{A}=B x_{1}\left(-\hat{x}_{1}+2 \hat{x}_{2}\right) / \sqrt{3}$, so that the magnetic translational operators are,

$$
S_{\boldsymbol{X}} \equiv e^{i e B m_{1} A \frac{\sqrt{3}}{2} x_{2}} T_{\boldsymbol{X}}
$$

where $T_{\boldsymbol{X}}$ are the geometrical translations. The commutation and multiplication rules are,

$$
S_{\boldsymbol{X}} S_{\boldsymbol{X}^{\prime}}=e^{i 2 \pi \frac{p}{q} m_{1}^{\prime} m_{2}} S_{\boldsymbol{X}+\boldsymbol{X}^{\prime}}, \quad S_{\boldsymbol{X}} S_{\boldsymbol{X}^{\prime}}=e^{i 2 \pi \frac{p}{q}\left(m_{1}^{\prime} m_{2}-m_{1} m_{2}^{\prime}\right)} S_{\boldsymbol{X}^{\prime}} S_{\boldsymbol{X}} .
$$

The subgroup of this group made of translations $\boldsymbol{R}=m_{1} q \boldsymbol{A}_{1}+m_{2} q \boldsymbol{A}_{2}$ on a $(q \times q)$-enlarged superlattice is isomorphic to a simple translational group, so that its eigenfunctions, $S_{R} \Phi_{k}=e^{i k \cdot \boldsymbol{R}} \Phi_{k}$, can be used as a plane wave basis. Since the whole group $\left\{S_{\boldsymbol{X}}\right\}$ is non-albelian, and has $q$-dimensional irreducible representations [66], the spectrum of such plane-wave states on the lattice will be $q$-fold degenerate. A convenient choice of basis states, to study Zak's miniband spectrum, is given by Bloch wavefunctions built from the Landau levels in Eq. (1.23),

$$
\Phi_{t, k}^{n, j}=\frac{1}{\sqrt{N}} \sum_{s} e^{-i k_{1} q A s} \psi_{n}^{k_{2}+\frac{\sqrt{3}}{2} b\left(p s+j+\frac{t p}{q}\right)}
$$

where the sums run over $r, s=-N / 2, \cdots, N / 2$. This basis is similar to the set of Bloch wavefunctions for a one dimensional chain with $p$ sites per elementary unit cell, labelled by $j=0, \cdots, p-1$, and the Landau level index, $n$, analogous to multiple atomic orbitals on each site. The index $t=0, \cdots q-1$ labels basis functions in the $q$-dimensional irreducible representation and $\boldsymbol{k}=k_{1} \hat{k}_{1}+k_{2} \hat{k}_{2}$, $\left|k_{i}\right|<\frac{\sqrt{3}}{4 q} b, \hat{k}_{i} \cdot \hat{x}_{j}=\delta_{i, j}$. The action of magnetic translations on this basis set,

$$
S_{\boldsymbol{A}_{1}} \Phi_{t, k}^{n, j}=\Phi_{t+1, \boldsymbol{k}}^{n, j}, \quad S_{\boldsymbol{A}_{2}} \Phi_{t, k}^{n, j}=e^{i \frac{2 \pi p t}{q}} e^{i k \cdot \boldsymbol{A}_{2}} \Phi_{t, k}^{n, j}
$$

determines both the degeneracy of, and the lack of coupling between, wavefunctions built from Bloch states with different $t$.

To calculate the magnetic miniband spectrum, we calculate the matrix ele- 


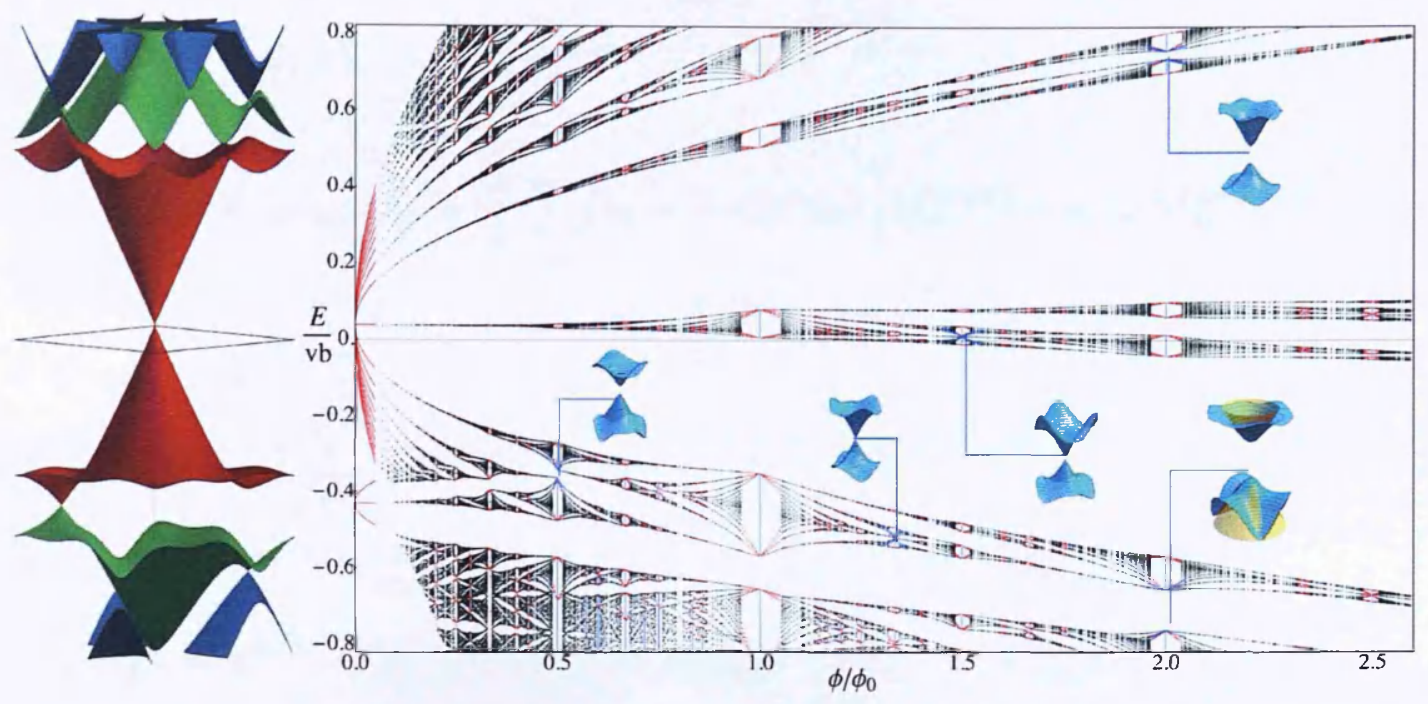

Figure 4.3: The numerical calculated energy spectrum in the absence of a magnetic field (left), and the magnetic miniband spectrum (right) calculated for $\boldsymbol{k}=0$ (black points), or, when the flux takes a simple fraction, the full spectral support (blue vertical lines). The insets show the magnetic miniband spectrum for simple fractions, with the fitting to a gapped Dirac spectra show in yellow for the lower magnetic miniband at $\phi / \phi_{0}=2$. [The right panel was provided by Xi Chen.]

ments,

$$
\left\langle\Phi_{t, k}^{n, j}|H| \Phi_{t^{\prime}, k^{\prime}}^{n^{\prime}, j^{\prime}}\right\rangle=\delta_{t, t^{\prime}} \delta_{k, k^{\prime}} \sum_{\tilde{s}} e^{-i k_{1}^{\prime} q a \tilde{s}} \mu_{n n^{\prime}}^{k_{2}+\frac{\sqrt{3}}{2} b j, k_{2}+\frac{\sqrt{3}}{2} b\left(p \tilde{s}+j^{\prime}\right)}
$$

of Hamiltonian (2.2) between Bloch states (4.2) with various $n$ and $j$, and numerically diagonalize the resulting Heisenberg matrix, with a large enough range of $n$ to ensure convergence of the resulting energy spectrum. Since Hamiltonian (2.2) contains only the simplest moiré harmonics, the sum in the above expression is 
limited to $\tilde{s}=0, \pm 1$

$$
\begin{aligned}
\mu_{n n^{\prime}}^{k_{2} k_{2}^{\prime}}= & \delta_{n, n^{\prime}} \delta_{k_{2}, k_{2}^{\prime}} E_{n}+\frac{v b}{2} \sum_{m}\left(u_{0}+i(-1)^{m} \tilde{u}_{0}\right)\left[M_{m}^{|n|,\left|n^{\prime}\right|}+s_{n} s_{n^{\prime}} M_{m}^{|n|-1,\left|n^{\prime}\right|-1}\right] \\
& -\frac{v b}{2} \sum_{m} \xi i\left(\tilde{u}_{1}+i(-1)^{m} u_{1}\right)\left[e^{\frac{i 2 \pi}{3}} s_{n} M_{m}^{|n|,\left|n^{\prime}\right|-1}+e^{\frac{-i 2 \pi}{3}} s_{n^{\prime}} M_{m}^{|n|-1,\left|n^{\prime}\right|}\right] \\
& +\frac{v b}{2} \sum_{m} \xi\left(\tilde{u}_{3}+i(-1)^{m} u_{3}\right)\left[M_{m}^{|n|,\left|n^{\prime}\right|}-s_{n} s_{n^{\prime}} M_{m}^{|n|-1,\left|n^{\prime}\right|-1}\right] ; \\
M_{0}^{n n^{\prime}}= & e^{-i \sqrt{3} \lambda^{2}} W_{5 \pi / 6,1}^{n n^{\prime}} \delta^{-}, M_{1}^{n n^{\prime}}=W_{-5 \pi / 6,0}^{n n^{\prime}} \delta^{-}, M_{2}^{n n^{\prime}}=W_{-\pi / 2,-1}^{n n^{\prime}} \delta^{0}, \\
M_{3}^{n n^{\prime}}= & e^{-i \sqrt{3} \lambda^{2}} W_{-\pi / 6,-1}^{n n^{\prime}} \delta^{+}, M_{4}^{n n^{\prime}}=W_{\pi / 6,0}^{n n^{\prime}} \delta^{+}, M_{5}^{n n^{\prime}}=W_{\pi / 2,1}^{n n^{\prime}} \delta^{0} ; \\
W_{c_{1}, c_{2}}^{n n^{\prime}} & =A_{n} A_{n^{\prime}} 2^{n} \sqrt{\pi} n^{\prime} ! L_{n^{\prime}}^{n-n^{\prime}}\left(2 \lambda^{2}\right) e^{-\lambda^{2}}\left(\lambda e^{i c_{1}}\right)^{n-n^{\prime}} e^{i c_{2} k_{2} 4 \lambda^{2} / b}, \quad n \geq n^{\prime} \geq 0 .
\end{aligned}
$$

Here $L_{n}^{n^{\prime}}$ is the associated Laguerre polynomial, $\lambda=b \lambda_{B} / 2, \delta^{ \pm}=\delta_{k_{2}^{\prime} k_{2} \pm \sqrt{3} b / 2}$, $\delta^{0}=\delta_{k_{2}^{\prime} k_{2}}$. The $k_{2}$ dependence has the period $p \sqrt{3} b / 2$.

\subsection{Generic Features in the Magnetic Miniband}

\section{Spectra}

We now discuss the generic features of the numerically calculated magnetic miniband spectrum shown in Fig. 4.3. In the left panel, we show the $\phi=0$ dispersion surfaces using parameters taken from the microscopic models (see chapter 2, Eq. (2.12)) with $v^{-}=\theta=0$ and $v^{+}=0.063 v b$. This spectrum contains both a gapless zero-energy DP and gapless secondary DP on the valence band side of the spectra. The right panel contains the energy spectrum calculated for rational values of the flux. For $\phi \lesssim 0.2 \phi_{0}$ the magnetic miniband spectra can still be traced to the sequence of Landau level (extended towards $\phi=0$ as red lines) corresponding to the two DPs.

At higher flux, the degeneracy of each Landau level is split by the moiré perturbation to reveal an intricate self-similar spectra, consisting of repeated Landau level-like features at multiple flux and at multiple energy scales. Despite this, the zeroth Landau level of the main DP remains separated from the rest of the 
spectrum by large gaps, which allow unambiguous identification of filling factors $\nu= \pm 2$. The carrier density (measured in carriers per moiré unit cell) required to place the chemical potential within this gap is obtained using $\rho_{0}=\nu \phi / \phi_{0}$. For all other gaps, the carrier density is determined by $\rho=\rho_{0}+s g_{s} g_{v} / q$, where $s$ is the number of magnetic minibands crossed from the reference density $\rho_{0}$, and $g_{s}=g_{v}=2$ accounts for spin and valley degeneracy. In the absence of time reversal symmetry, that is $\phi \neq 0$, the valley degeneracy in Fig. 4.3 is only prescribed by the spatial inversion symmetry, and is absent if the inversion asymmetric superlattice perturbation is included *.

When the flux ratio is simple, that is integers $p$ and $q$ are small, the magnetic minibands have a significant band width. Representative examples are shown as insets in the right panel of Fig. 4.3, and generally take the form of a weakly gapped DP with spectrum $\epsilon \approx \sqrt{v_{\text {eff }}^{2} p^{2}+\Delta^{2}}$. For a small window of flux $\phi=p \phi_{0} / q+\delta \phi$, the magnetic miniband spectra can then be traced [73] to a sequence of effective Landau levels, obtained from the weakly gapped Dirac-like spectra, using Eq. (1.23), and relative magnetic field $\delta B=\delta \phi / S$. This allows us to obtain an approximation to the magnetic minibands spectrum (blue continuous lines in Fig. 4.3) in the vicinity of simple flux fractions, where the dimension of the Heisenberg matrix with basis (4.2) is too large to diagonalize. Each effective Landau level undergoes its own splitting by the moiré perturbation, although the zeroth effective Landau level generally remains well separated. Also note the visible discontinuity in the energy of the zeroth effective Landau level as it skips across the gap, $\Delta$, at $\delta B=0$.

In the main and lower left panel of Fig. 4.4, we show the fan diagram in which each gap in Fig. 4.3 is mapped to the point at the carrier density at which the gap occurs, with a strength of colour reflecting the gap size. This figure provides our main contact with forthcoming compressibility measurements, since the presence of each significant gap, $\Delta>0$, will be observed as an incompressible state.

${ }^{*}$ For $\tilde{u}_{i}=0$, the spatial inversion symmetry, $H(\boldsymbol{r}, \zeta)=\sigma_{z} H(-\boldsymbol{r},-\zeta) \sigma_{z}$, prescribes the relation $\epsilon_{K_{+}+k}=\epsilon_{K_{-}-k}$ between spectra in graphene's two valleys. For $\phi=0$, time reversal symmetry prescribes the same relation, however when both $\phi, \tilde{u}_{i} \neq 0$ the spectra in the two valleys are unrelated. 
In Fig. 4.4 the well separated zeroth Landau levels associated with both the zero-energy DP at $\rho=0$, and the secondary DP at $\rho=-4$ produce clearly visible filling factor lines $\nu= \pm 2$. Similarly, each Dirac-like feature, occurring at simple flux ratio $\phi / \phi_{0}=p / q$, generates its own peculiar crossing features in fan diagram 4.4. Here the $g_{s} g_{v} q$-fold degeneracy of the well separated zeroth effective Landau level prescribes a pair of crossing, strongly gapped, filling factor lines separated by tilt angle $g_{s} g_{v} q$. These features are seen at many locations on Fig. 4.4 including those which correspond to the exemplary magnetic miniband spectra shown on Fig. 4.3. Moreover, these features are independent of the particular realization of the moiré perturbation.

In the lower right panel of Fig. 4.4, the fan diagram is shown for the same inversion symmetric moiré perturbation as the rest of the figure but with the additional inversion asymmetric perturbation $v^{-}=v^{+} / 10$. This eliminates the valley degeneracy, $g_{v}$, which is manifested in the fan diagram as additional filling factor lines intersecting at tilt angles $g_{v} q$. Note that similar effects, including the lifting of spin degeneracy, may be provided by the electron-electron interaction.

\subsection{Conclusion}

We diagonalize the phenomenological Hamiltonian of graphene with a hBN underlay, in a strong magnetic field. The resulting spectral support shows robust features, independent of the particular realization of the moiré perturbation, which arises due to the tendancy of the magnetic minibands to take the form of gapped Dirac points. The degeneracy of effective Landau levels, traced to these features, is reflected in the change of tilt angle of filling factor lines in the fan diagram. 


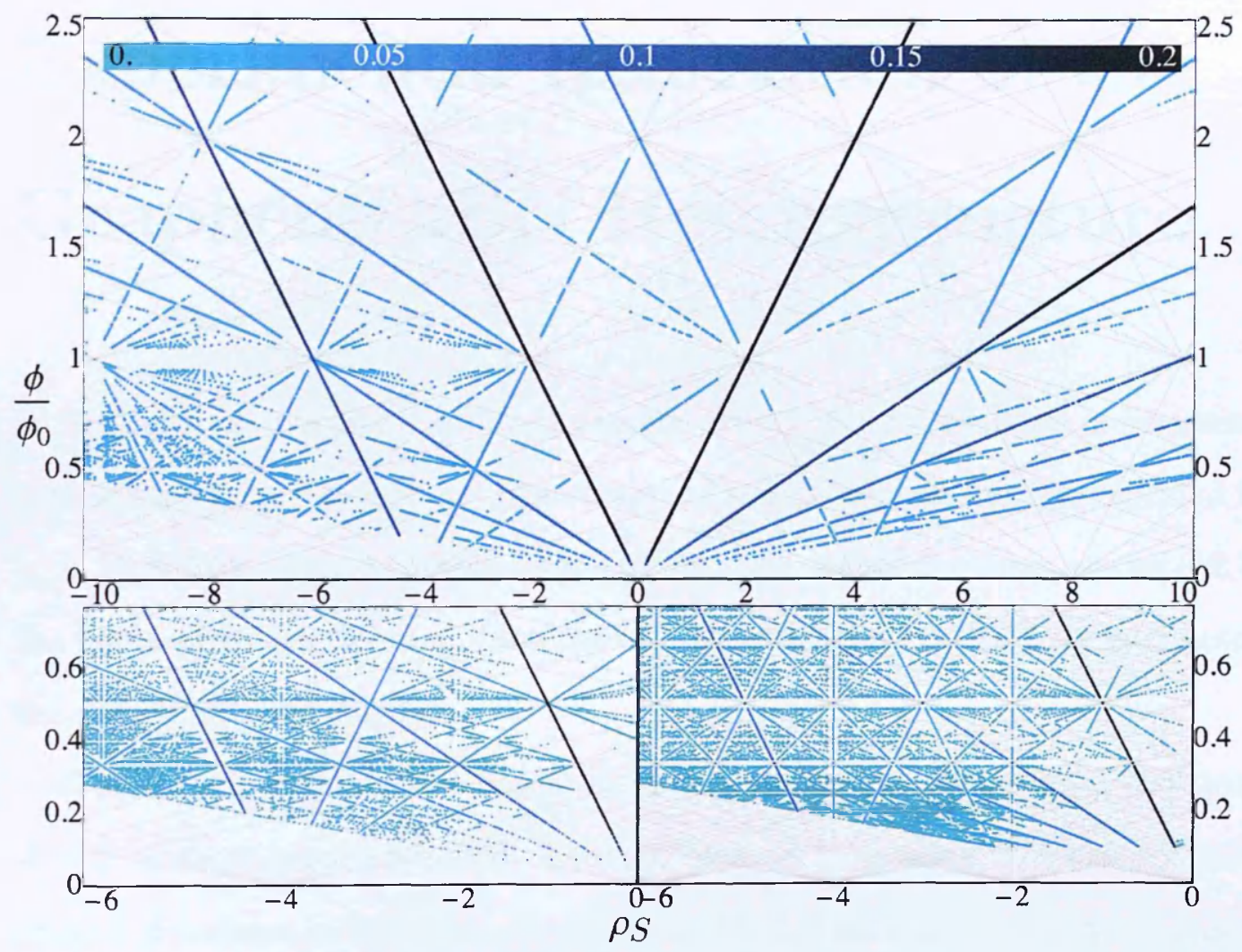

Figure 4.4: The fan diagram for the same moiré perturbation as Fig. 4.3 (top) with a detailed enlargement of the section containing the valence band secondary DP (lower left). The lower right panel shows the same region, but the inversion asymmetric part of the moiré perturbation has been included. The grey lines fulfil the Diophantine equation $[71,72] ; \nu=\sigma_{H}+g_{s} g_{v} s \phi_{0} / \phi, \sigma_{H}=g_{s} g_{v} z+2, s$ and $z$ are integers. [Figure provided by $\mathrm{Xi}$ Chen.] 


\section{Chapter 5}

\section{Experimental Realisation of the \\ Graphene-hBN Heterostructure}

In this short chapter we describe the recent experimental realisation of graphenehBN heterostructures, and the observation of the Hofstadter spectra, reported by L. A. Ponomarenko and co-workers [23]. The experimental work was carried out by the group of Andre Geim, and interpreted using the theory described in chapters the preceding three chapters.

The experimental devices consisted of encapsulated multi-terminal Hall bars, similar to those reported in [12]. The new element here being that the crystallographic directions of the graphene flake and the bottom $\mathrm{hBN}$ layer were aligned to within about one degree. A second hBN layer was used to encapsulate the graphene flake. This top hBN layer was deliberately misaligned from the graphene to avoid additional low-energy reconstructions of the graphene spectra. An oxidised Si wafer acted as a back gate, enabling a shift in graphene's Fermi energy of up to $\sim 0.35 \mathrm{eV}$. This allowed access to the part of the spectrum reconstructed by the superlattice perturbation.

Figure 5.1a shows the behaviour of longitudinal and Hall resistivities ( $\rho_{x x}$ and $\rho_{x y}$, respectively) for experimental device A. The standard peak in $\rho_{x x}$ at carrier density $n=0$, corresponds to graphene's main neutrality point. An additional 
a)

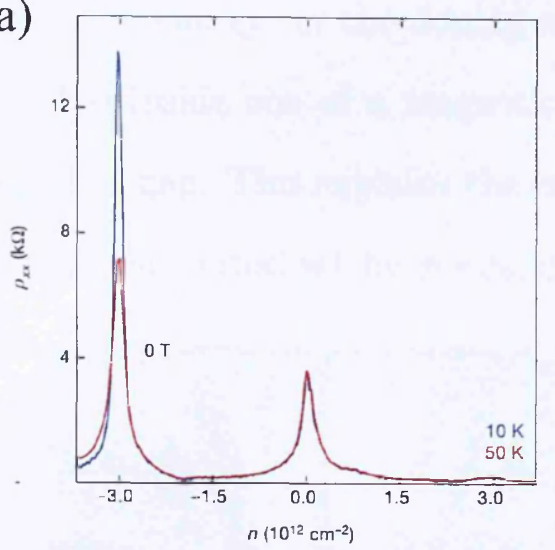

b)

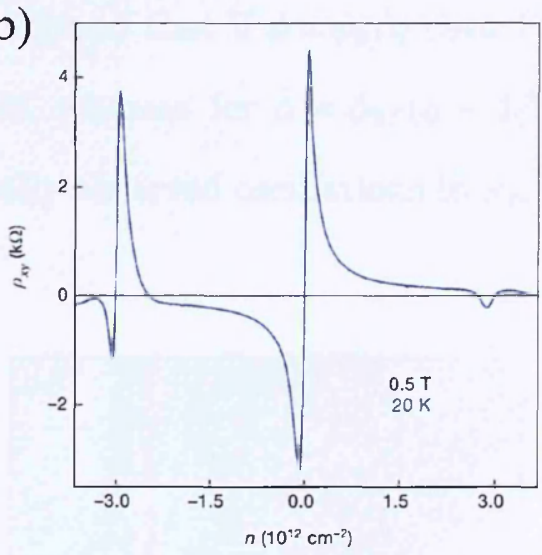

Figure 5.1: a) The Longitudinal resistivity, $\rho_{x x}$, as a function of $n$. Positive and negative values of $n$ correspond to electrons and holes, respectively. b) The experimentally measured Hall resistivity, $\rho_{x y}$, changes sign at hole doping $n_{S} \approx 3.0 \times 10^{12} \mathrm{~cm}^{-2}$, revealing a well-isolated secondary Dirac point. The data is for device A. [Adapted from Fig. 1 Ref. [23]]

peak in $\rho_{x x}$ appears at the doping $n=n_{S}$ of four holes per moiré unit cell. This is accompanied by a reversal in sign of $\rho_{x y}$ (Fig. 5.1b). These features prove that electron-like carriers appear in the valence bands of graphene, and are attributed to the isolated secondary Dirac points discussed in section 2.3. Similar features are found in the conduction band, but are much weaker, and provide an example of the electron-hole symmetry breaking also discussed in section 2.3 .

In Figure 5.2 the observed fan diagrams are presented. Near doping $n=$ $n_{S}$, the Hall resistivity repeatedly changes its sign with increasing $B$, indicating recurrent appearance and disappearance of electron-like orbits within graphene's valence band (Fig. 5.2 b). This occurs with a periodicity in $1 / B$ described by $\phi=\phi_{0} / q$. The calculated spectral support allows us to understand many of the experimentally observed features. Indeed, Figure $5.2 \mathrm{c}$ shows strong features at fluxes $\phi=\phi_{0} / q$, which are attributed to the gapped Dirac-like magnetic miniband spectra (see section 4), and give rise to sign changes in Hall resistivity. The effective Landau levels arising from these magnetic minibands are illustrated in Fig. 5.2d by magenta curves, which, as an example, magnifies a part of Fig. 5.2c near the hole side neutrality point and $\phi=\phi_{0} / 2$.

The capacity of the magnetic minibands can be used to determine the position 
of the Fermi energy for the doping $n=n_{S}$. It is found that if $\phi=\phi_{0} / q$ then Fermi energy lies inside one of a magnetic minibands, whereas for $\phi=\phi_{0} /(q+1 / 2)$ it lies inside a gap. This explains the experimentally observed oscillations in $\sigma_{x x}(B)$, with have the period set by $\phi=\phi_{0} / q$.
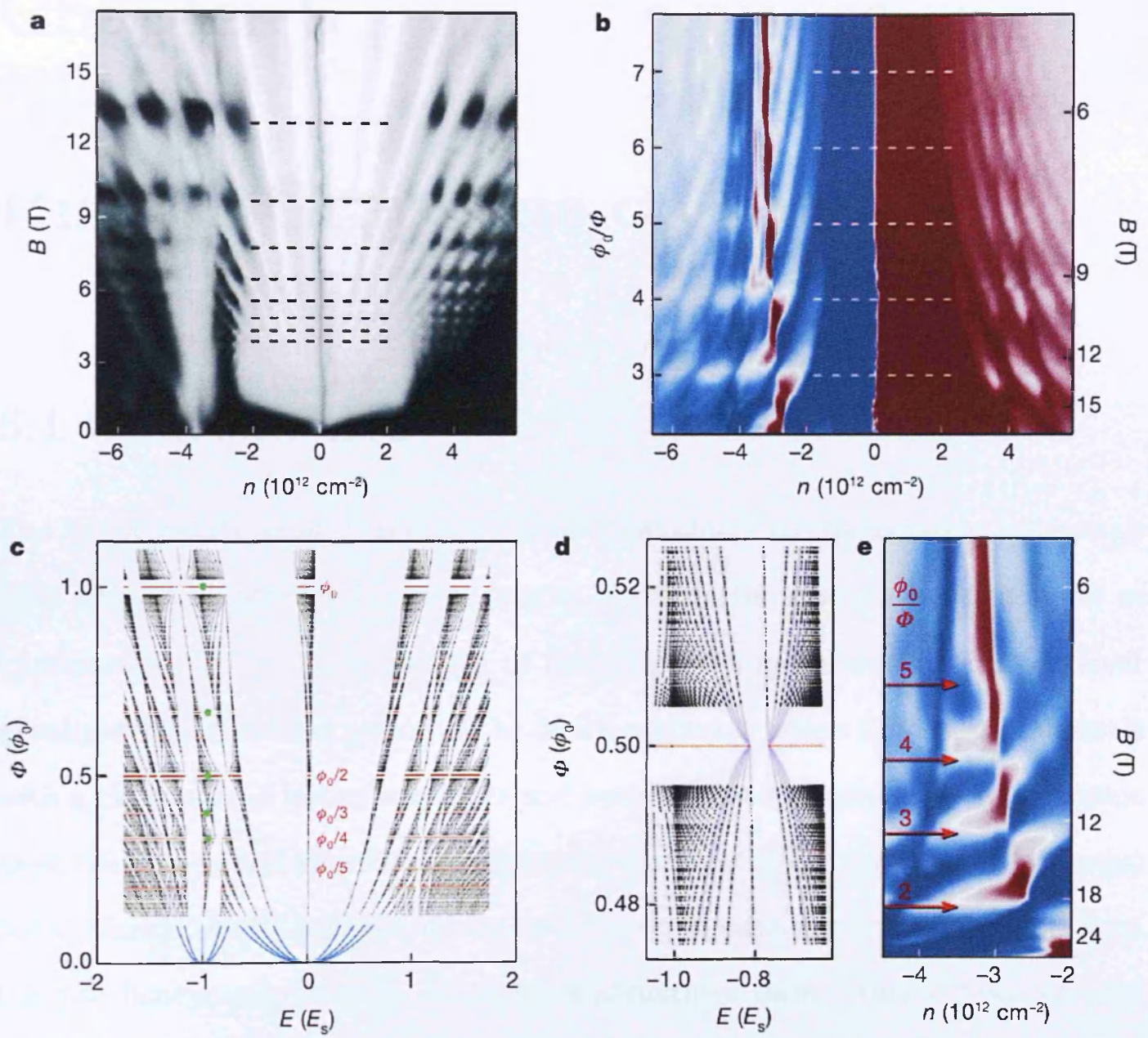

Figure 5.2: a) Experimentally measured longitudinal conductivity, $\sigma_{x x}=\rho_{x x} /\left(\rho_{x x}^{2}+\right.$ $\rho_{x y}^{2}$ ), as a function of $n$ and $B$ (device B). Grey scale: white, $0 \mathrm{k} \Omega$; black, $2.2 \mathrm{~m} S$. The dashed lines indicate $B=(1 / q) \phi_{0} / A_{u c}$ with $q=3-10$. b) Experimentally measured Hall resistivity, $\rho_{x y}$, for the same device, as a function of $n$ and $1 / B$. Colour scale: navy, $-2 \mathrm{k} \Omega$; white, $0 \mathrm{k} \Omega$; wine, $2 \mathrm{k} \Omega$. In both plots, $T=2 \mathrm{~K}$. c) Spectral support for the graphene-hBN heterostructure. The blue curves show several low index Landau levels calculated from the $\phi=0$ miniband spectra. The green dots indicate the position of Fermi level for $n=n_{S}$. d) Section of (c) with superimposed effective Landau levels calculated from the $\phi=\phi_{0} / 2$ magnetic miniband spectra. e) Hall resistivity, $\rho_{x y}$, for device $\mathrm{C}\left(n_{S} \approx 3.6 \times 10^{12} \mathrm{~cm}^{-2}\right)$. Colour scale: navy, $-3 \mathrm{k} \Omega$; white, $0 \mathrm{k} \Omega$; wine, $3 \mathrm{k} \Omega$. $T=20 \mathrm{~K}$. [Taken from Fig. 3 Ref. [23]] 


\section{Chapter 6}

\section{Bilayer Graphene on hBN}

\subsection{Introduction}

The heterostructures of graphene with other hexagonal layered crystals, or crystals with hexagonal symmetry facets, feature moiré patterns which are the result of incommensurability of the periods of the two two-dimensional lattices, or their misalignment. Since the period of the moiré pattern is longer for a pair of crystals with a closer size of lattice constants and better aligned principal crystallographic axes, the long period moiré superlattices are characteristic for graphene/hexagonal boron nitride (hBN) heterostructures with a small misalignment angle $\theta$ between the two honeycomb lattices. Such heterostructures have recently been created by transferring graphene onto hBN [10, 22, 23, 25]. The influence of hexagonal moiré patterns on Dirac electrons in monolayer graphene (MLG) has been studied in detail $[10,18,19,22,23,25,74]$, using both specific microscopic models and phenomenologically. Three possible types of moiré miniband structures on the conduction/valence band sides of graphene's spectrum have emerged from the theories $[18,19,22,23,25,74]$ : sometimes, spectra without a distinct separation between the lowest and other minibands; quite exceptionally, the first miniband separated from the next band by a triplet of secondary Dirac points (sDPs) in each of the graphene valleys $K_{+}$and $K_{-}$; more generically, a single sDP at the edge of the first miniband in each valley. Also, the signatures of the miniband 
formation have been observed experimentally in the tunnelling density of states [22] and magnetotransport characteristics [23, 25] in MLG/hBN heterostructures.

In this chaper, we analyse the characteristic moiré miniband features in heterostructures of bilayer graphene (BLG) with highly oriented and almost commensurate, hexagonal crystals, such as hBN, recently created and investigated using magneto-transport measurements by Dean et al. [24]. We find that, in contrast to monolayers, the electronic spectrum of BLG on hBN is most likely to exhibit gaps between the first moiré miniband and the rest of the spectrum (on valence or conduction band side, and sometimes in both bands), or have the bands strongly overlapping with each other, whereas Dirac points at the miniband edge appear only for exceptional choices of moiré parameters. Also, we find that a gap at the edge between the valence and conduction bands can be opened in BLG by the same moiré perturbation that would not open a 'zero-energy' gap in MLG. This behaviour is prescribed by the substrate creating a moiré perturbation only for one layer of BLG, thus breaking the inversion symmetry of the moiré superlattice. The results of a systematic study of the miniband regimes in BLG-hBN heterostructures is summarized in the parametric space diagrams in Fig. 6.1, where the regions of the parameter space with gapped spectra are painted red and overlapping (non-resolved) bands are left transparent. Differences between the two diagrams corresponding to different misalignment angles $\theta$ arises from the interplay between the orientation of the supercell Brillouin zone (sBZ) and the skew interlayer hopping in BLG. This interplay, unique to BLG, can help in narrowing down the microscopic parameters of moiré pattern at graphene-hBN interface using magnetotransport and capacitance experiments.

\subsection{Moiré Superlattice Hamiltonian}

The analysis in this chapter is performed using the phenomenological approach of chapter 2. This involves the description of the long-range moiré superlattice using a Dirac-type model for graphene electrons, where we include all symmetry-allowed 

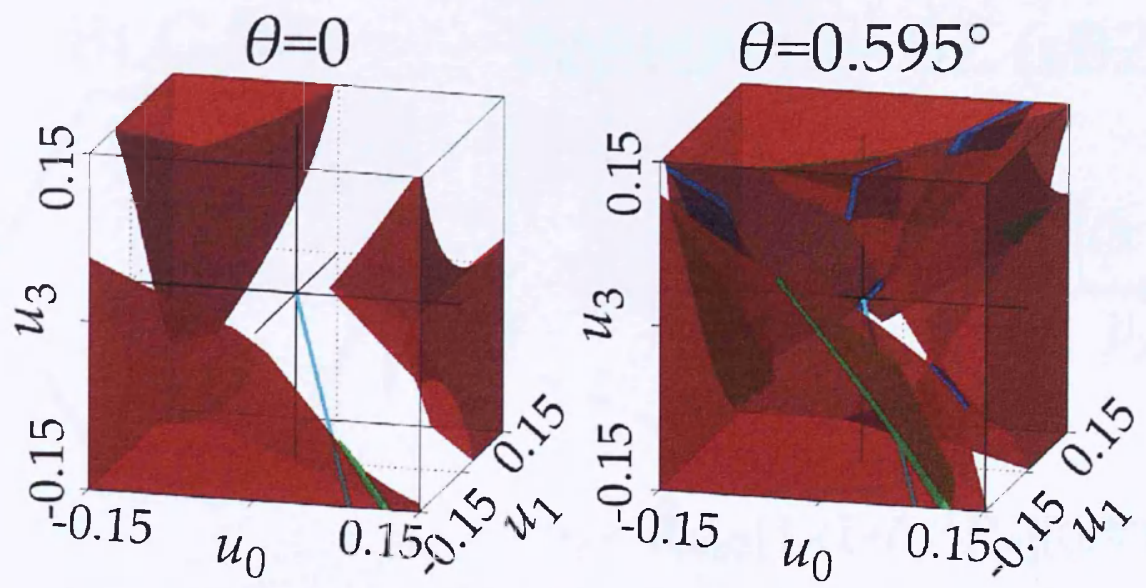

Figure 6.1: Parameter space $\left(u_{0}, u_{1}, u_{3}\right)$ used to classify characteristic behaviour of moire miniband in highly oriented BLG on almost commensurate substrate: in red, we paint regions where BLG spectrum has a gap separating the first miniband in the valence band from the rest of the spectrum, regions with overlapping (unresolved) bands are transparent and blue/green mark the degenerate conditions for the appearance of isolated secondary Dirac points at the first miniband edge, like in MLG [18, 19, 22]. The thick cyan lines show directions in the parameter space favoured by the point charge lattice and graphene-hBN hopping models discussed in chaper 2. A similar parametric plot describing the minibands on the conduction band side can be obtained by inversion in the $u_{0}-u_{3}$ plane.

terms in the moiré perturbation applied to one of the two layers in BLG and perform an exhaustive numerical analysis in order to characterise the miniband behaviour over a broad range of the parameter space. For BLG placed on top of a substrate with hexagonal symmetry and the lattice constant $a_{\mathrm{S}}=(1+\delta) a$ larger by $\delta$ than that of graphene, $(a=2.46 \AA$, and for the case of $h \mathrm{BN}, \delta=1.8 \%[10])$, the lattice mismatch, together with a possible misalignment of the two lattices given by the angle $\theta$, lead to a periodic structure which can be described using a set of reciprocal lattice vectors,

$$
\boldsymbol{b}_{n}=\hat{\boldsymbol{R}}_{n \pi / 3}\left[1-(1+\delta)^{-1} \hat{\boldsymbol{R}}_{\theta}\right]\left(0, \frac{4 \pi}{\sqrt{3} a}\right), \quad n=0,1, \ldots, 6
$$

where $\hat{\boldsymbol{R}}_{\varphi}$ stands for anticlockwise rotation by angle $\varphi$ and $b=\left|\boldsymbol{b}_{n}\right| \approx \frac{4 \pi}{\sqrt{3} a} \sqrt{\delta^{2}+\theta^{2}}$ [25]. Note that this set both rotates by $\phi(\theta)$ and changes its size as a function of $\theta$, Fig. 6.2. Because of the rapidly decaying nature of the interlayer interaction, we only take into account the influence of $\mathrm{hBN}$ on the bottom carbon layer, neglecting any interaction with the top one. Then, following the MLG investiga- 


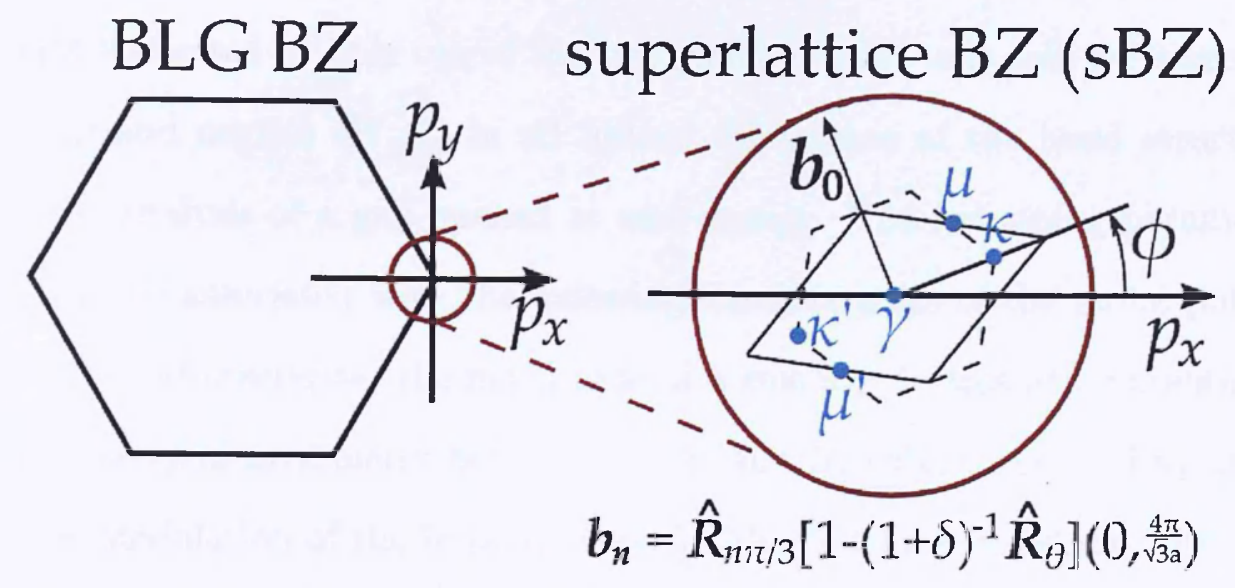

Figure 6.2: Hexagonal Brillouin zone (BZ) of BLG with the valley coordinate system $p_{x}, p_{y}$ and the electronic bands in the vicinity of the $K$ valley, together with the zoom in on the supercell Brillouin zone (sBZ) where we mark its own symmetry points $\kappa$ and $\mu$.

tions of chapter 2 , the electrons in $\mathrm{BLG} / \mathrm{hBN}$ heterostructure are described by the Hamiltonian

$\hat{\boldsymbol{H}}=\left(\begin{array}{cc}v \boldsymbol{\sigma} \cdot \boldsymbol{p}+\delta \hat{\boldsymbol{H}}_{\mathrm{sym}}+\delta \hat{\boldsymbol{H}}_{\mathrm{asym}} & \frac{\gamma_{1}}{2}\left(\tau_{3} \sigma_{1}-i \sigma_{2}\right)+\frac{v_{3}}{2}\left(\sigma_{1}+i \tau_{3} \sigma_{2}\right)\left(p_{x}+i \tau_{3} p_{y}\right) \\ \frac{\gamma_{1}}{2}\left(\tau_{3} \sigma_{1}+i \sigma_{2}\right)+\frac{v_{3}}{2}\left(\sigma_{1}-i \tau_{3} \sigma_{2}\right)\left(p_{x}-i \tau_{3} p_{y}\right) & v \boldsymbol{\sigma} \cdot \boldsymbol{p}\end{array}\right)$ $\delta \hat{\boldsymbol{H}}_{\mathrm{sym}}=u_{0} v b f_{1}(\boldsymbol{r})+u_{3} v b f_{2}(\boldsymbol{r}) \sigma_{3} \tau_{3}+u_{1} v\left[\boldsymbol{l}_{\boldsymbol{z}} \times \nabla f_{2}(\boldsymbol{r})\right] \cdot \boldsymbol{\sigma} \tau_{3}+u_{2} v \nabla f_{2}(\boldsymbol{r}) \cdot \boldsymbol{\sigma} \tau_{3}$, $\delta \hat{\boldsymbol{H}}_{\text {asym }}=\tilde{u}_{0} v b f_{2}(\boldsymbol{r})+\tilde{u}_{3} v b f_{1}(\boldsymbol{r}) \sigma_{3} \tau_{3}+\tilde{u}_{1} v\left[\boldsymbol{l}_{\boldsymbol{z}} \times \nabla f_{1}(\boldsymbol{r})\right] \cdot \boldsymbol{\sigma} \tau_{3}+\tilde{u}_{2} v \nabla f_{1}(\boldsymbol{r}) \cdot \boldsymbol{\sigma} \tau_{3}$, $f_{1}(\boldsymbol{r})=\sum_{n} e^{i \boldsymbol{b}_{n} \cdot \boldsymbol{r}}, \quad f_{2}(\boldsymbol{r})=i \sum_{n}(-1)^{n} e^{i \boldsymbol{b}_{n} \cdot \boldsymbol{r}}$,

written in the basis of the Bloch states on sublattices $\left\{\Phi\left(A_{1}\right), \Phi\left(B_{1}\right), \Phi\left(A_{2}\right), \Phi\left(B_{2}\right)\right\}$ in the $K_{+}$valley and $\left\{\Phi\left(B_{1}\right),-\Phi\left(A_{1}\right), \Phi\left(B_{2}\right),-\Phi\left(A_{2}\right)\right\}$ in $K_{-}$, where indices $1 / 2$ mark the bottom/top layers and the substrate directly acts on the electrons in the bottom layer. We also use $\hbar=1$ and employ two sets of Pauli matrices $\sigma_{i}$, $\boldsymbol{\sigma}=\left(\sigma_{1}, \sigma_{2}\right)$, and $\tau_{i}$, acting in the sublattice and valley space, respectively.

The moiré perturbation, scaled using energy scale $v b$ and parametrised using dimensionless $\left\{u_{i}, \tilde{u}_{i}\right\}, i=0,1,2,3$, captures the effect of the substrate on BLG through its coupling with carbon orbitals in the closest, bottom layer only. Following the approach used in the monolayer study, we separate the moiré perturbation due to hBN into inversion-symmetric and asymmetric terms, $\delta \hat{\boldsymbol{H}}_{\text {sym }}$ and $\delta \hat{\boldsymbol{H}}_{\text {asym }}$, respectively. By considering two limiting cases in which electrons in graphene are 
dominantly affected by only one of the two atoms in hBN unit cell, we argue that $\left|\tilde{u}_{i}\right| \ll\left|u_{i}\right|$ and neglect $\delta \hat{\boldsymbol{H}}_{\text {asym }}$ in all further discussions of the band structures, except the analysis of a gap opened at zero energy. The remaining parameters, $u_{0,1,2,3}$, can be associated with the following characteristics of the moiré pattern: parameter $u_{0}$ characterizes the magnitude of a smooth electrostatic potential, $u_{3}$ captures the local asymmetry between the $A_{1}$ and $B_{1}$ sublattices, and $u_{1}$ and $u_{2}$ introduce modulation of the in-plane hops for the electrons travelling within the bottom layer. As opposed to the monolayer case, in BLG the rotorless $u_{2}$ term cannot be completely gauged away. However, it vanishes for zero misalignment angle, and its effect on the band structure is generated via the interplay with the trigonal warping term: therefore, it is small and can be neglected. Note that, since we scale all energies by $v b \approx v \frac{4 \pi}{\sqrt{3} a} \sqrt{\delta^{2}+\theta^{2}}$, the size of dimensionless parameters $u_{i}$ in Eq. (6.1) would be larger for smaller angle $\theta$ for the same pair of BLG and a substrate.

\subsection{Symmetries in the Miniband Structure}

Due to the time-inversion symmetry $[38,77]$, described in chapter 1

$$
\hat{\boldsymbol{H}}(\boldsymbol{p})=\sigma_{2} \tau_{2}\left[\hat{\boldsymbol{H}}^{*}(-\boldsymbol{p})\right] \sigma_{2} \tau_{2}
$$

electronic spectra in the two valleys are related, $\epsilon_{\boldsymbol{K}_{+}+\boldsymbol{p}}=\epsilon_{\boldsymbol{K}_{-}-\boldsymbol{p}}$, so that we only discuss electronic spectra the $K_{+}$valley. We find that, in contrast to unperturbed BLG, the spectrum resulting from a generic choice of parameters in Hamiltonian (6.1) is not electron-hole symmetric, but obeys the following relation,

$$
\epsilon_{K_{+}+\boldsymbol{p}}^{u_{0}, u_{1}, u_{3}}=-\epsilon_{K_{+}+\boldsymbol{p}}^{-u_{0}, u_{1},-u_{3}}
$$

which reduces the parameter space $\left(u_{0}, u_{1}, u_{3}\right)$ to be explored. 


\subsection{Low-energy Hamiltonian for BLG-hBN Het-}

\section{erostructures}

For the sake of a systematic comparison with the low-energy 'two-band' model of free-standing BLG [35], applicable at the energy scale $\epsilon \ll \gamma_{1}$, we use a SchriefferWolff transformation [43] and project the four-band Hamiltonian onto the lowenergy bands, reducing it to an effective two-band Hamiltonian,

$$
\begin{aligned}
& \hat{\boldsymbol{H}}_{\mathrm{eff}}=-\frac{v^{2}}{\gamma_{1}}\left[\left(p_{x}^{2}-p_{y}^{2}\right) \sigma_{1}+2 p_{x} p_{y} \sigma_{2}\right] \tau_{3}+v_{3}(\boldsymbol{\sigma} \cdot \boldsymbol{p})^{T}+ \\
& \frac{v b}{2} g_{+}(\boldsymbol{r})\left(1+\sigma_{3} \tau_{3}\right)+\frac{v^{3} b}{2 \gamma_{1}^{2}}\left(p_{x}-i \sigma_{3} p_{y}\right) g_{-}(\boldsymbol{r})\left(p_{x}+i \sigma_{3} p_{y}\right)\left(1-\sigma_{3} \tau_{3}\right)+ \\
& \frac{v^{2} b}{2 \gamma_{1}}\left[\left(p_{x}+i p_{y} \tau_{3}\right) g(\boldsymbol{r})\left(\sigma_{1}-i \sigma_{2} \tau_{3}\right)+g^{*}(\boldsymbol{r})\left(p_{x}-i p_{y} \tau_{3}\right)\left(\sigma_{1}+i \sigma_{2} \tau_{3}\right)\right]
\end{aligned}
$$

where

$$
\begin{aligned}
& g_{ \pm}(\boldsymbol{r})=\left(u_{0} \pm \tilde{u}_{3}\right) f_{1}(\boldsymbol{r}) \pm\left(u_{3} \pm \tilde{u}_{0}\right) f_{2}(\boldsymbol{r}) \\
& g(\boldsymbol{r})=\sum_{n} e^{i \boldsymbol{b}_{n} \cdot \boldsymbol{r}}\left(\hat{b}_{n}^{x}+i \hat{b}_{n}^{y} \tau_{3}\right)\left[(-1)^{n}\left(u_{2}+i u_{1} \tau_{3}\right)+\left(\tilde{u}_{1} \tau_{3}-i \tilde{u}_{2}\right)\right] \\
& \boldsymbol{b}_{n}=b\left(\hat{b}_{n}^{x}, \hat{b}_{n}^{y}\right) .
\end{aligned}
$$

The applicability of the simplified Hamiltonian to the description of, at least, the first moiré miniband in the BLG spectrum requires that $\gamma_{1} \gtrsim 2 v b$. For a perfectly aligned BLG/hBN heterostructure, we estimate that $\frac{\gamma_{1}}{v b} \approx \frac{\sqrt{3}(1+\delta)}{4 \pi} \frac{a \gamma_{1}}{v}=1.107$, which suggests that a quantitative description of moiré minibands in $\mathrm{BLG} / \mathrm{hBN}$ requires the use of the four-band Hamiltonian, Eq. (6.1). 


\subsection{Miniband Spectra of BLG-hBN Heterostruc-}

\section{tures}

Little is known about the exact values of each of the perturbation parameters $u_{i}$. Due to the size of the moiré pattern and the importance of Van der Waals interaction in graphene/hBN heterostructures, the resources needed for supercellsize ab initio calculations are prohibitive. At the same time, even for the more studied system of $\mathrm{MLG} / \mathrm{hBN}$, experimental data obtained so far also does not allow for clear determination of the perturbation [23]. Hence, in our numerical modeling of spectra, we broadly cover the $\left(u_{0}, u_{1}, u_{3}\right)$ parameter space, Fig. 6.1. Examples of characteristic moiré miniband spectra on the valence band side in BLG/hBN are shown in Fig. 6.3: (a) overlapping minibands characteristic for the transparent part of the parameter space in Fig. 6.1; (b) and (c) gapped spectrum at the edge of the first moire miniband characteristic for the red-painted part of the parameter space in Fig. 6.1. Also shown are the corresponding densities of states, with a global gap in the valence band, Fig. 6.3(b), (c).

Spectra in Fig. 6.3(a) and (c) both correspond to the same choice of perturbation, $u_{0}=u_{1}=0, u_{3}=0.15$, but with a different misalignment angle $\theta$, which is enough to cause opening of a band gap between the first and second minibands on the valence side. This is because of the trigonal warping of the unperturbed BLG spectrum, which shifts the energy $\epsilon_{\boldsymbol{p}}^{0}$ of a momentum state $\boldsymbol{p}=\left(p_{x}, p_{y}\right)$ by $\epsilon_{\text {warp }} \approx-s v_{3} p \cos 3 \varphi$, where $\varphi=\arctan \frac{p_{y}}{p_{x}}, p=\sqrt{p_{x}^{2}+p_{y}^{2}}$ and $s=1(s=-1)$ denotes the conduction (valence) band. For $\theta=\phi=0$, this results, for example, in the $\kappa\left(\kappa^{\prime}\right)$ point in the valence band shifted up (down) in energy, like in Fig. 6.3(a). However, misalignment angle $\theta=0.595^{\circ}$ leads to sBZ rotation by $\phi=-30^{\circ}$ and the trigonal warping correction at the points $\kappa$ and $\kappa^{\prime}$ of the rotated sBZ vanishes, as seen in Fig. 6.3(c). Such a strong dependence of the miniband spectrum on the misalignment angle is special to BLG, because in MLG trigonal warping corrections are much weaker. Consequently, experimental investigation of the BLG 
miniband spectra for several misalignment angles (determined from the moiré geometry) may yield new information about the nature of the perturbation felt by graphene electrons due to hBN. For example, two of the models suggested for the MLG/hBN heterostructure, graphene-hBN hopping model and point charge lattice model (see chaper 2), yield the same form of the $u_{i}$ coefficients as a function of the misalignment angle $\theta$,

$$
u_{0}=\frac{1}{2} \tilde{v}, \quad u_{1}=-\frac{\delta}{\sqrt{\delta^{2}+\theta^{2}}} \tilde{v}, \quad u_{3}=-\frac{\sqrt{3}}{2} \tilde{v}
$$

( $\tilde{v}>0$ is a strength of the perturbation), corresponding to a single line for each misalignment angle $\theta$, as shown with bold cyan lines in Fig. 6.1 for $\theta=0$ and $\theta=0.595^{\circ}$. It is interesting to note that in the diagram for $\theta=0.595^{\circ}$, this line passes close behind the green region where a secondary Dirac point at $\kappa^{\prime}=\left(-\frac{1}{\sqrt{3}} b, 0\right)$ separates the first and the second miniband on the valence side. In Fig. 6.4, we show a detailed study of the gap between the first and second miniband in the conduction band as a function of the misalignment angle $\theta$ and perturbation magnitude $u=\sum_{i}\left|u_{i}\right|$, assuming that Eq. (6.3) holds, where 'negative' gap means that the minibands overlap. However, for the valence band, due to trigonal warping, a gap opens for $\theta \approx 0.6^{\circ}$; for points marked with a circle, this is a direct gap at the $\kappa^{\prime}$ point. Note that even a small change of misalignment angle leads to a large rotation of the moiré pattern and a different involvement of trigonal warping due to skew interlayer coupling in BLG. The corresponding evolution of BLG moiré spectra is illustrated in Fig. 6.5. For $\theta=0$, left column in Fig. 6.5, trigonal warping affects $\kappa$ and $\kappa^{\prime}$ in the opposite fashion (shown in the inset) which obscures the $\mathrm{sDP}$ at $\kappa^{\prime}$. However, the sDP becomes isolated on the energy scale for $\theta \approx 0.5^{\circ}$, centre column in Fig. 6.5. It disappears again as the misalignment angle is increased further, with the asymmetry between $\kappa$ and $\kappa^{\prime}$ reaching a maximum for $\theta=1.786^{\circ}$ when the sBZ is rotated by $60^{\circ}$, right column in Fig. 6.5.

Also, all of our above conclusions have been tested for a "single-side gate" geometry, where large charge density required to fill the first miniband (four elec- 
trons per moiré supercell) is induced by a single gate, which induces additional interlayer asymmetry [78, 79], and found that its presence has almost no effect on the type of the miniband spectrum at the edge of the first miniband (gapped or overlapping minibands).

One can notice that, in the majority of the spectra presented in Fig. 6.3 and 6.5, and for generic moiré perturbation, a gap, $\Delta$, is opened at the 'neutrality point' between the conduction and valence bands, in contrast to monolayer graphene, where a such gap, $\Delta_{0}^{\mathrm{MLG}}=24 v b\left|u_{1} \tilde{u}_{0}+u_{0} \tilde{u}_{1}\right|$, appears only when moiré pattern contains an inversion asymmetric perturbation. In the monolayer, this is accompanied by an overall shift, $12 v b\left(u_{1} u_{3}+\tilde{u}_{1} \tilde{u}_{3}\right)$, resulting in the edges of conduction/valence band at $\epsilon_{ \pm}^{\mathrm{MLG}}=12 v b\left(u_{1} u_{3}+\tilde{u}_{1} \tilde{u}_{3}\right) \pm 12 v b\left(u_{1} \tilde{u}_{0}+u_{0} \tilde{u}_{1}\right)$. In BLG, one of the two degenerate zero-energy states belongs to the bottom and one to the top layer. Because the top layer is unaffected by the perturbation, the corresponding state stays at zero energy, while the other one is shifted by $\epsilon_{+}^{\mathrm{MLG}}$, just like one of the states in MLG. As a result, the bilayer gap is nonzero even if the moiré perturbation is inversion-symmetric,

$$
\Delta^{\mathrm{BLG}} \approx 12 v b\left|u_{1}\left(u_{3}+\tilde{u}_{0}\right)+\tilde{u}_{1}\left(u_{0}+\tilde{u}_{3}\right)\right|
$$

as a consequence of the $\mathrm{hBN}$ substrate breaking the equivalence of the two layers and, hence, the inversion symmetry. For BLG sandwiched between two hBN layers, the asymmetry will be still present, if the misalignment angles between graphene and top/bottom $\mathrm{hBN}$ layers are different.

\subsection{Conclusion}

We shown that the interplay between interlayer coupling (including skew hopping between layers leading to the trigonal warping effect in BLG) in bilayer graphene and breaking of layer symmetry by the substrate play an important role in determining the miniband spectrum of $\mathrm{BLG}-\mathrm{hBN}$ heterostructures. As opposed to 
MLG in which a gap at the Dirac point is open only for inversion-asymmetric moiré perturbation, in BLG a 'zero-energy' gap is open even for an inversionsymmetric perturbation, as a direct consequence of interlayer asymmetry caused by the substrate. 


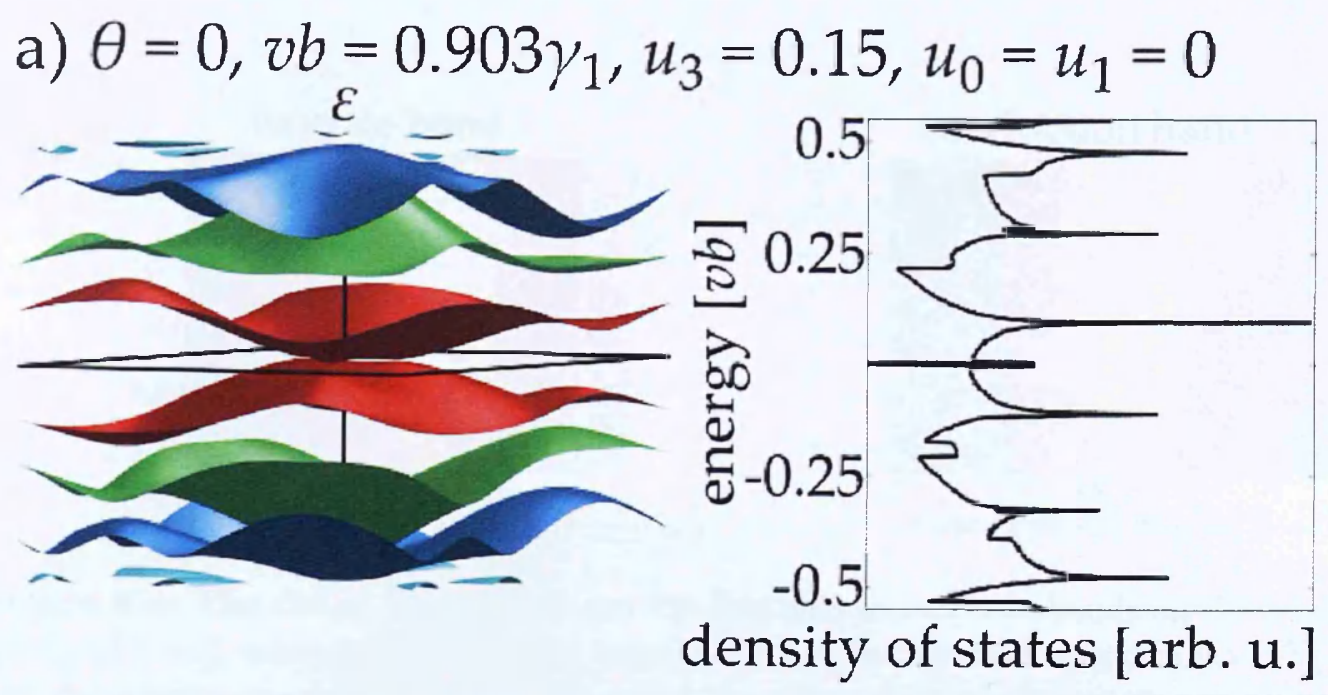

b) $\theta=0, v b=0.903 \gamma_{1}, u_{0}=0.15, u_{1}=u_{3}=0$
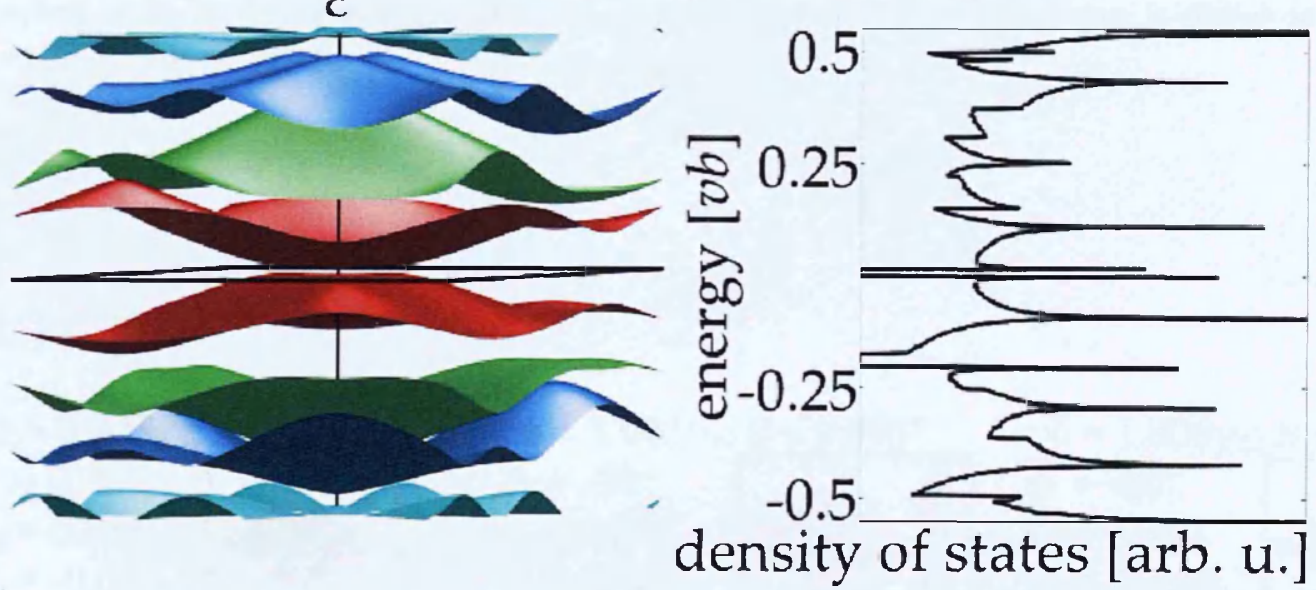

c) $\theta=0.595^{\circ}, v b=1.053 \gamma_{1}, u_{3}=0.15, u_{0}=u_{1}=0$
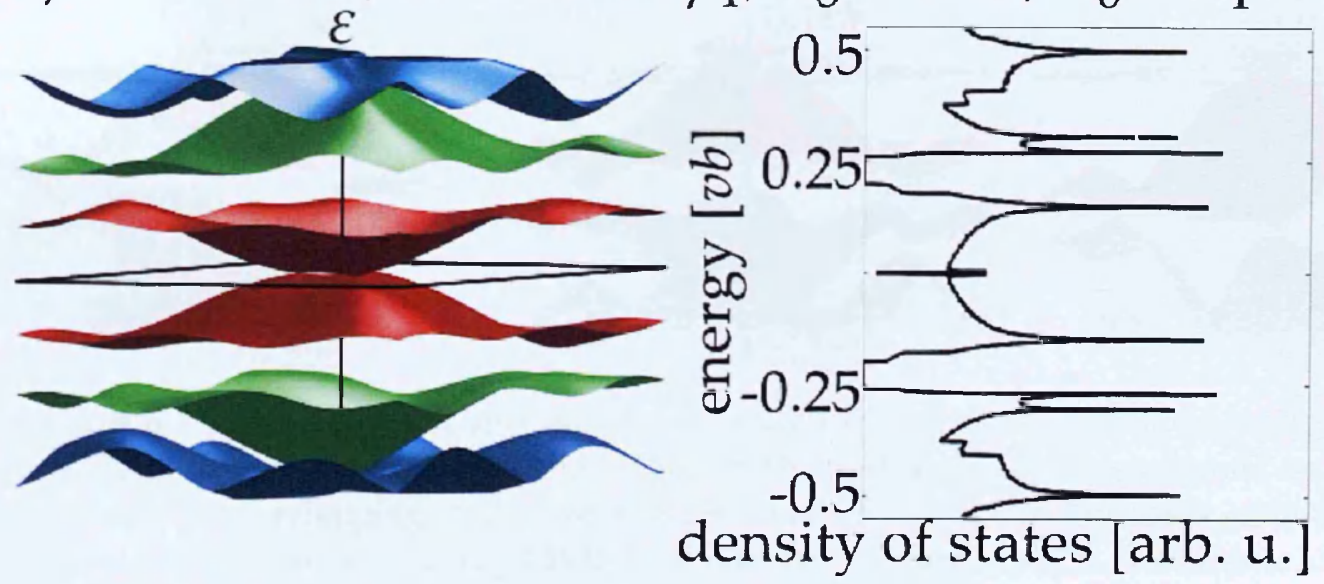

Figure 6.3: (a-c) Moiré miniband spectra (drawn within the rhombic sBZ) and density of states (DoS) portraying two characteristic behaviours of the miniband spectrum as determined in Fig. 6.1. 

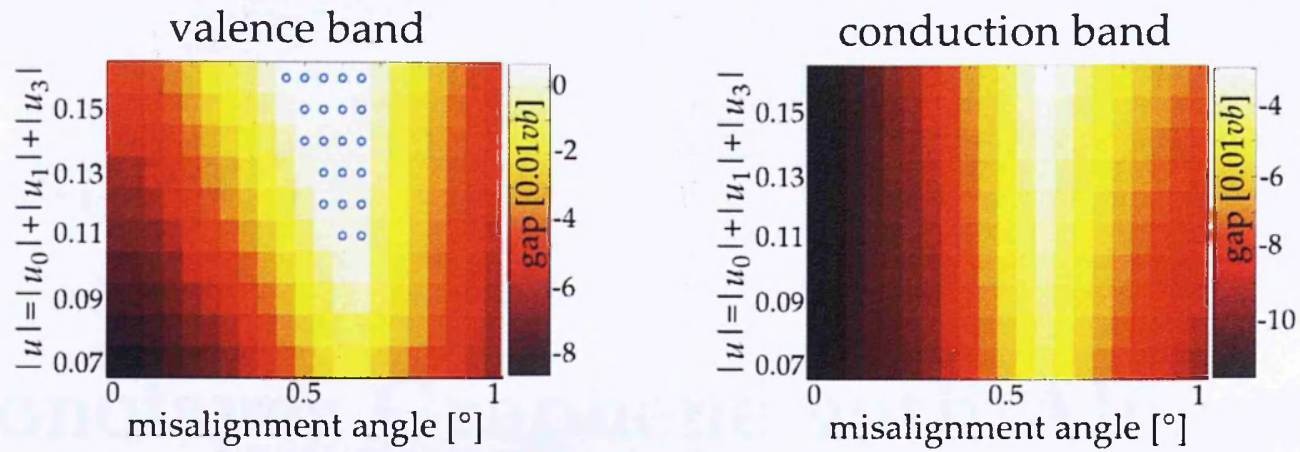

Figure 6.4: The size of the gap between the first and second minibands on the conduction (right) and valence (left) side as a function of the perturbation magnitude $u=\sum_{i}\left|u_{i}\right|$ and the misalignment angle $\theta$, assuming that the perturbation parameters are described by relations in Eq. (3). Negative values for the gap denote overlapping bands. Points marked with blue circles correspond to spectra for which the global gap is direct at $\kappa^{\prime}$.
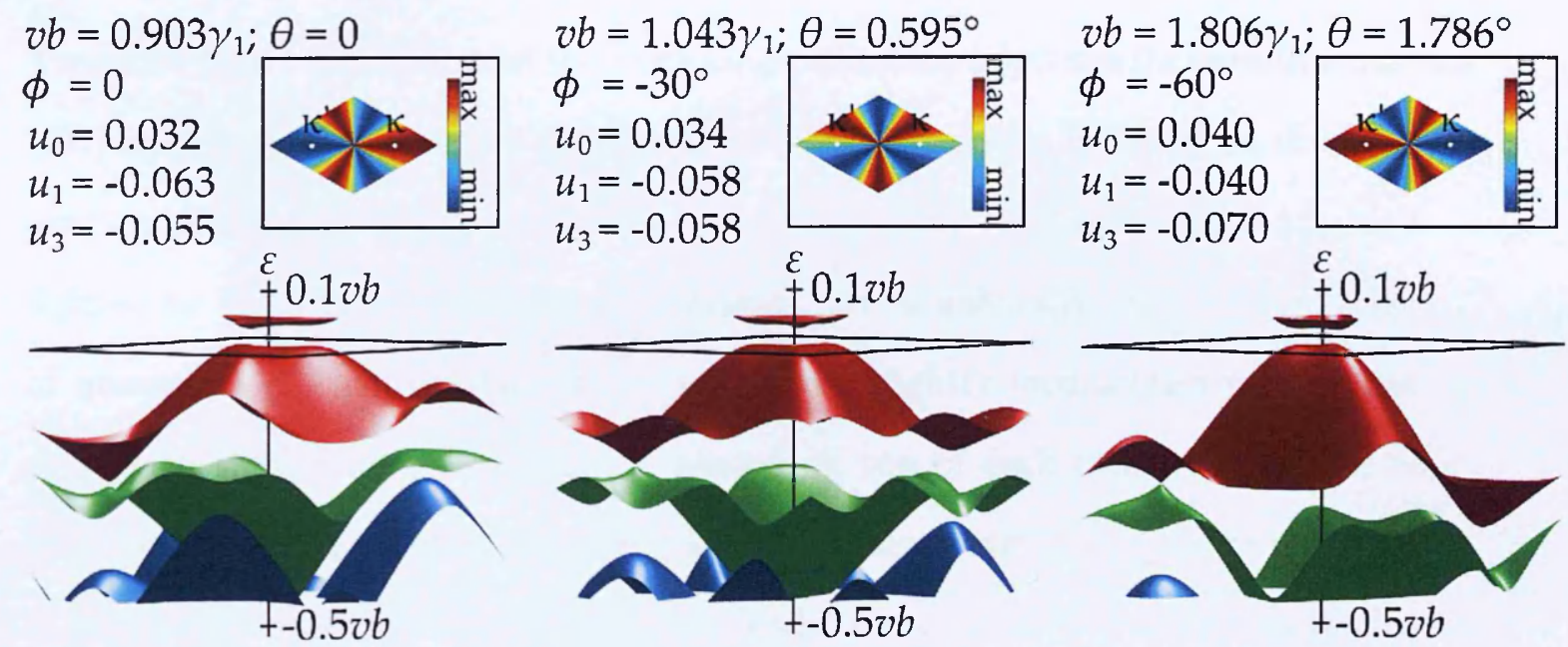

Figure 6.5: Moiré miniband spectra (drawn within the rhombic sBZ) illustrating the role of trigonal warping in determining whether the first and second minibands on the valence side are overlapping or gapped at a secondary Dirac point. For these examples, we assumed that relations in Eq. (3) hold and set $u=\sum_{i}\left|u_{i}\right|=0.15$. The insets show the angular dependence, $\cos 3 \phi$, of the trigonal warping for the valence band within the (rotated) sBZ. 


\section{Chapter 7}

\section{Monolayer Graphene with Almost}

\section{Commensurate $\sqrt{3} \times \sqrt{3}$}

\section{Hexagonal Crystals}

\subsection{Introduction}

Two alternative methods exist to create long-period superlattices for two-dimensional (2D) electrons. One method, developed for semiconductors, is based on the lithographic patterning of the semiconductor surface [68]. The other method, highlighted by the studies of $2 \mathrm{D}$ atomic crystals, arises naturally from the existence of quasi-periodic moiré patterns formed by two slightly incommensurate 2D lattices with similar crystal symmetry, placed on top of each other. Graphene on hexagonal boron nitride is one example of such heterostructure, where the effect of the moiré superlattice on $2 \mathrm{D}$ electrons leads to pronounced changes in the electronic properties detected by STM [11, 80, 81], and magnetotransport experiments [23-25].

The specific form of moiré superlattice for graphene electrons, generated by a hexagonal underlay, depends on the ratio between the periods of the two lattices and their mutual orientation. The abundance of layered hexagonal crystals 

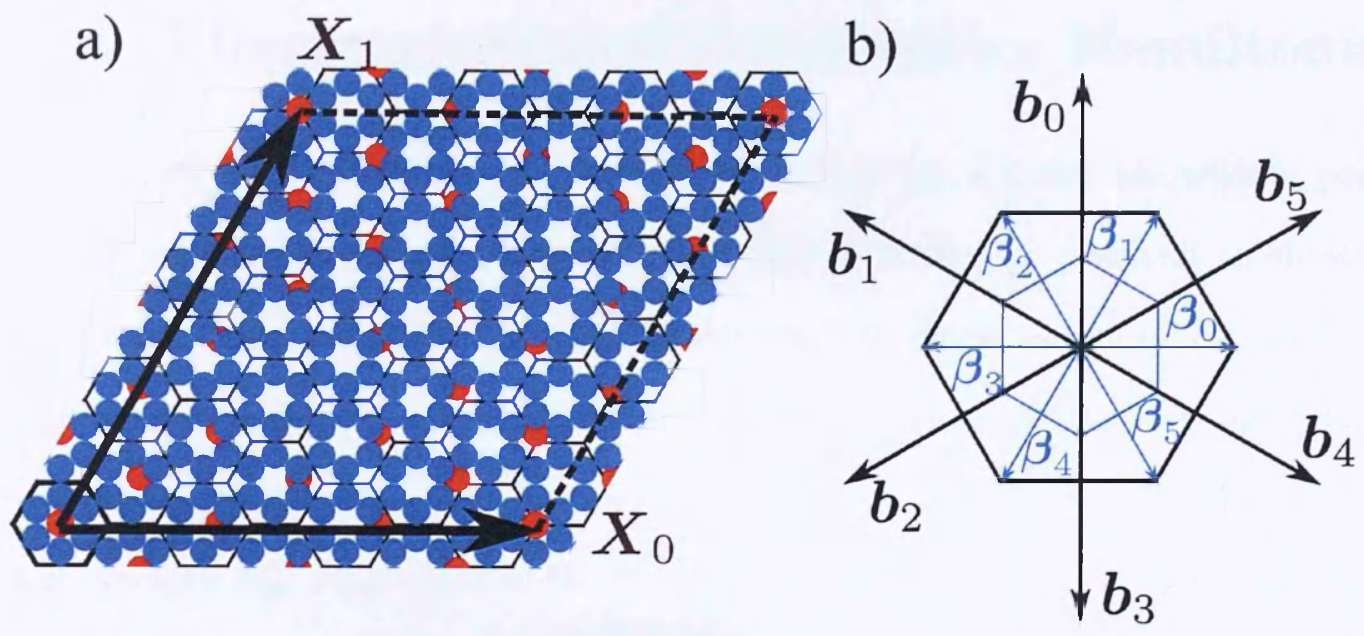

Figure 7.1: (a) The moire pattern formed from graphene (blue) on a underlay (red) with $\theta=0, \delta=\frac{1}{9}$. The black hexagons follow Kekulé lattice of graphene. (b) The two sets of reciprocal lattice vectors, $\boldsymbol{b}_{m}$ and $\boldsymbol{\beta}_{m}$, with their associated Brillouin zones.

and semiconductors with a hexagonal surface layer, allows for a multiplicity of qualitatively different superlattice structures, with various levels of moiré supercell complexity. The simplest and, by now, best studied is the highly orientated graphene-hBN heterostructure. Here we analyze the second simplest moiré pattern for Dirac electrons in graphene produced by a hexagonal underlay with an elementary unit cell approximately 3 times bigger than that of graphene. The effect of a perfectly commensurate $\sqrt{3} \times \sqrt{3}$ superlattice, known as the Kekulé distortion of the honeycomb lattice [82], consists in the Bragg type intervalley scattering of graphene electrons, which opens a gap between the conduction and valence bands. A hexagonal underlay with the lattice constant $a_{S}=\sqrt{3}(1+\delta) a$, $|\delta| \ll 1$, slightly different from that of the Kekulé superlattice of graphene and a small misaligned angle $\theta$, produce a periodically oscillating intervalley coupling. Although this does not open a gap in graphene's Dirac point, it creates a specific miniband spectrum, whose generic features are studied in this chapter. Below, we employ a phenomenological approach to classify the possible structure of moiré minibands of Dirac electrons in graphene [21] and, in particular, the behaviour of the edge of the first minibands on the conduction and valence band sides. 


\subsection{Phenomenological Superlattice Hamiltonian}

The image of a moiré supperlattice for graphene on a substrate with a period almost commensurate with the $\sqrt{3} \times \sqrt{3}$ Kekulé lattice of graphene is shown in Fig. 7.1. Since graphene electrons belong to the Bloch states in its hexagonal Brillouin zone corners and a Kekulé perturbation leads to their intervalley Bragg scattering, the symmetry of the electronic system is described by the group of wavevectors $K_{ \pm}$, equivalent to the extended point group $C_{6 v}+t C_{6 v}+t^{2} C_{6 v}$ where $t$ is an elementary translation of the honeycomb lattice. That is why in Fig. 7.1 (a) we show both the actual positions of carbon atoms in graphene and, using lines, the Kekulé lattice. The periodic occurrence of sites from the underlay under equivalent positions of graphene honeycomb lattice is described by a moiré pattern which is periodic under translations by $X_{0}$ and $X_{1}$. The associated reciprocal lattice vectors belong to the set $\check{\boldsymbol{b}}=\left\{\boldsymbol{b}_{m}=\hat{R}_{2 \pi m / 6} \boldsymbol{b}_{0}\right\}_{m=0, \cdots, 5}$ where $\hat{R}_{\psi}$ is the rotation matrix, and $\boldsymbol{b}_{0}=\left[1-(1+\delta)^{-1} \hat{R}_{\theta}\right]\left(0, \frac{4 \pi}{\sqrt{3} a}\right)$, so that $\left|\boldsymbol{b}_{n}\right| \equiv b=\frac{4 \pi}{\sqrt{3} a} \sqrt{\delta^{2}+\theta^{2}}$. In contrast, the equivalent positions of substrate sites on the Kekule lattice are characterized by the $\sqrt{3}$ times longer period of $X_{0}+X_{1}$ and reciprocal lattice vectors from the set $\check{\boldsymbol{\beta}}=\left\{\boldsymbol{\beta}_{m}=\frac{1}{\sqrt{3}} \hat{R}_{\frac{-\pi}{2}} \boldsymbol{b}_{m}\right\}_{m=0, \cdots, 5}$ with $\left|\boldsymbol{\beta}_{n}\right| \equiv \beta=b / \sqrt{3}$. The coexistence of these two periodicities is taken into account, on an equal footing, in the phenomenological Hamiltonian for graphene's Dirac electrons,

$$
\begin{aligned}
\hat{H} & =v \hat{\boldsymbol{p}} \cdot \boldsymbol{\sigma}+U_{E^{\prime}} v \beta F(\check{\boldsymbol{\beta}}) \sigma_{3}+U_{G} v\left[\boldsymbol{\sigma} \times \boldsymbol{l}_{z}\right] \cdot \nabla F(\check{\boldsymbol{\beta}})+U_{G^{\prime}} v \boldsymbol{\sigma} \cdot \nabla F(\check{\boldsymbol{\beta}}) \\
& +u_{0} v b f_{1}(\check{\boldsymbol{b}})+u_{3} v b f_{2}(\check{\boldsymbol{b}}) \sigma_{3} \tau_{3}+u_{1} v\left[\boldsymbol{l}_{z} \times \nabla f_{2}(\check{\boldsymbol{b}})\right] \cdot \boldsymbol{\sigma} \tau_{3}+u_{2} v \tau_{3} \boldsymbol{\sigma} \cdot \nabla f_{2}(\check{\boldsymbol{b}}) \\
f_{1}(\check{\boldsymbol{v}}) & =\sum_{m=0, \cdots, 5} e^{i \boldsymbol{v}_{m} \cdot \boldsymbol{r}}, \quad f_{2}(\check{\boldsymbol{v}})=i \sum_{m=0, \cdots, 5}(-1)^{m} e^{i \boldsymbol{v}_{m} \cdot \boldsymbol{r}}, \quad F(\check{\boldsymbol{v}})=f_{1}(\check{\boldsymbol{v}}) \tau_{1}+f_{2}(\check{\boldsymbol{v}}) \tau_{2} .
\end{aligned}
$$

This Hamiltonian is written in terms of the Pauli matrices $\sigma_{i}$ and $\tau_{j}$ which act separately on the sublattice $(A, B)$ and valley $\left(K_{+}, K_{-}\right)$components of the 4spinors $\left(\psi_{A K_{+}}, \psi_{B K_{+}}, \psi_{B K_{-}},-\psi_{A K_{-}}\right)^{T}$ describing graphene electrons. Hence, the second line describes intravalley Bragg scattering, whereas the first line accounts for intervalley scattering. In writing $\hat{H}$, we use the earlier observation $[18,19,22$, 
51-53] that the potential felt by the graphene electrons is smoothened by the larger separation between graphene and the substrate than the carbon-carbon distance in graphene. For graphene on hBN, as well as twisted bilayer graphene, this resulted in the presence of only the simplest set of harmonics, $\check{b}$, in the moiré perturbation $[18,19,22,51-53]$. For graphene on a almost commensurate $\sqrt{3} \times \sqrt{3}$ hexagonal underlay the same argument leads to the appearance of the intervalley terms. In Eq. (7.1), the relative strength of moiré perturbations, measured in the unit of energy $v b=\sqrt{3} v \beta$, is set by dimensionless parameters $U_{E^{\prime}}, U_{G}, U_{G^{\prime}}, u_{i=0,1,2,3}$. Here, we assume that such moiré perturbation is small, $\left|U_{i}\right| \ll 1,\left|u_{j}\right| \ll 1$, and that the underlay has an inversion-symmetric unit cell, which is a natural approximation * for a simple monoatomic surface layer.

\subsection{Microscopic Models}

To supplement the phenomenological approach to describe the moiré supperlattice, Eq. (7.1), we also estimated parameter $U_{i}$ and $u_{j}$ by adapting the two microscopic models discussed in section 2.6. That is, where the underlay is modelled as a hexagonal lattice of point charges (section 2.6.1), or modelled as a lattice of atomic orbitals on to which the graphene electrons can hop (section 2.6.2). Both models produce similar estimates for sets of phenomenological parameters $U_{i}$ and $u_{j}$,

$$
\begin{aligned}
& v \beta\left\{U_{E^{\prime}}, U_{G}, U_{G^{\prime}}\right\}=V\left\{\frac{1}{2}, \frac{-\delta}{\sqrt{\delta^{2}+\theta^{2}}}, \frac{\theta}{\sqrt{\delta^{2}+\theta^{2}}}\right\} \\
& v b\left\{u_{0}, u_{1}, u_{2}, u_{3}\right\}=v\left\{\frac{1}{2}, \frac{-\delta}{\sqrt{\delta^{2}+\theta^{2}}}, \frac{\theta}{\sqrt{\delta^{2}+\theta^{2}}},-\frac{\sqrt{3}}{2}\right\} .
\end{aligned}
$$

*Inversion asymmetric terms may be included into Eq. (7.1) by adding terms with $f_{1}(\check{\boldsymbol{v}}) \rightarrow$ $f_{2}(-\check{\boldsymbol{v}})$ and $f_{2}(\check{\boldsymbol{v}}) \rightarrow f_{1}(-\check{\boldsymbol{v}})$. 
Parameters $V$ and $v$ reflect the strength of the intervalley and intravalley perturbations. For the point charge model,

$$
\begin{gathered}
V=I(4 \pi /(3 a)), \quad v=I(4 \pi /(\sqrt{3} a)) \\
I(g) \approx \frac{8 \pi|\mathrm{e}| Q}{9 a^{4} \epsilon} \int d q_{z} d q_{z}^{\prime} \frac{\psi^{*}\left(\boldsymbol{K}, q_{z}\right) e^{i\left(q_{z}-q_{z}^{\prime}\right) \cdot d} \psi\left(\boldsymbol{K}, q_{z}^{\prime}\right)}{g^{2}+\left(q_{z}-q_{z}^{\prime}\right)^{2}}
\end{gathered}
$$

Here $Q$ is the charge per substrate lattice site, $D$ the graphene-substrate distance, $\epsilon$ the electric permittivity and $\psi\left(\boldsymbol{Q}, q_{z}\right)$ the Fourier transform of the graphene $\mathrm{P}^{z}$ orbitals $^{\dagger}$. The point charge model predicts $\tilde{V} \gg \tilde{v}$, whereas for the hopping model $V=v=\frac{-\gamma^{2}}{9 \epsilon_{s}}$ with $\gamma$ the hopping integral to the substrate and $\epsilon_{s}$ is the energy of the substrate state.

\subsection{Parameter Space of the Superlattice Pertur-}

\section{bation}

The features of the miniband spectrum of the Dirac electrons prescribed by the intravalley terms, $u_{j}$, in the second line of Eq. (7.1) have already been explored in chapter 2 as well as in previous studies of graphene on $h B N[18,19,22]$. The characteristic features, present in the low energy graphene band structure for this case, consist in the formation of additional secondary Dirac points $[18,21,22]$ in a gapless spectrum. $\ddagger$ In contrast, intervalley perturbations $U_{i}$ are able to open gaps in the spectrum at the edges of the low energy moiré minibands. Hence, we focus on the role of the intervalley terms, and explore the parameter space $\left[U_{E^{\prime}}, U_{G}, U_{G^{\prime}}\right]$, classifying the resulting electron spectra. It is useful to notice that

\footnotetext{
${ }^{\dagger}$ Comparison with section 2.6 suggest, all things being equal, that the point charge model of the $\sqrt{3} \times \sqrt{3}$ underlay generates a significantly stronger perturbation than $1 \times 1$ underlay. This follows from the fact that $I(g)$ decreases rapidly as a function of its argument.

¥The addition of a small intervalley component to a larger intravalley perturbation may open a minigap in the secondary DP, depending upon which valley the secondary DP occurs in.
} 


$$
-\epsilon_{-U_{E^{\prime}}, U_{G}, U_{G^{\prime}}}(\boldsymbol{k})=\epsilon_{U_{E^{\prime}}, U_{G}, U_{G^{\prime}}}(\boldsymbol{k})=\epsilon_{-U_{E^{\prime}},-U_{G},-U_{G^{\prime}}}(\boldsymbol{k}) .
$$

The first equality in Eq. (7.3) allows us to relate the bandstructure of the valence band to that of the conduction band by flipping the sign of $U_{E}$. Also, it turns out that the parameter $U_{G^{\prime}}$ affects the miniband spectra of electrons only in the second order, since its first order effect on the electron energies can be removed by the gauge transformation $\boldsymbol{\psi} \rightarrow e^{-i U_{G^{\prime}} F(\check{\boldsymbol{\beta}})} \psi^{\prime}$.

\subsection{Translational Symmetries and Zone Folding}

The correspondence between the translational symmetries of the Hamiltonian $\hat{H}$ and the geometrical symmetry group of the moiré supperlattice, $G_{S L}=\left\{c_{6}, T_{\boldsymbol{X}_{0}}\right\}$, is set by the fact that a translation e.g. by the period $\boldsymbol{X}_{0}$ indicated in Fig. 7.1, is accompanied by a valley-dependent unitary gauge transformation, $\hat{U}_{t}=-\frac{1}{2}-\frac{\sqrt{3} i}{2} \tau_{3}$, which represents the effect of the elementary translation of the honeycomb lattice on the 4-component spinors $\psi$. This argument establishes the isomorphism of $G_{S L}$ to the symmetry group $G_{H}=\left\{\hat{c}_{6}, \hat{S}_{\boldsymbol{X}_{0}}\right\}$ of the Hamiltonian $\hat{H}$, where, instead of geometrical translation $T_{\boldsymbol{X}_{0}}$, we use $\hat{S}_{\boldsymbol{X}_{0}}=\hat{U}_{t} \hat{T}_{\boldsymbol{X}_{0}}$ (and $\hat{S}_{\boldsymbol{X}_{1}}=\hat{U}_{t}^{\dagger} \hat{T}_{\boldsymbol{X}_{1}}$ instead of $T_{\boldsymbol{X}_{1}}$ ). This correspondence allows one to use two equivalent descriptions of the folded mini Brillouin zone (mBZ) of the electrons in the presence of the moiré pattern, Fig. 7.1(b). One, based on the longer periodicity implicit in the $e^{i \boldsymbol{\beta}_{m} \cdot \boldsymbol{r}}$ dependence of the intervalley part of the Hamiltonian $\hat{H}$, suggests plotting the miniband dispersion over the smaller $\mathrm{mBZ}$. The other, adjusted to the periodicity of the geometrical arrangement of atoms, uses the three times larger mBZ. For the smaller mBZ, the Dirac cones from both $K_{+}$and $K_{-}$valleys are folded onto the centre of the $\mathrm{mBZ}$, resulting in the valley degenerate dispersion surfaces shown in the left panel of Fig. 7.2(a). In contrast, the zone folding into the larger $\mathrm{mBZ}$, shown in the centre panel, places Dirac cones from graphene's two valleys 
at opposite mBZ corners. The folding of dispersion surfaces from the larger mBZ into the smaller $\mathrm{mBZ}$ can be used relate the spectra shown in these alternative schemes. The unfolding of smaller mBZ into the larger $\mathrm{mBZ}$ is provided by the gauge transformation $\boldsymbol{\psi} \rightarrow U \boldsymbol{\psi}^{\prime}, \hat{H} \rightarrow \hat{H}^{\prime}=U^{\dagger} \hat{H} U$ where $U=e^{\frac{i}{2}\left(\boldsymbol{b}_{0}+\tau_{3} \boldsymbol{\beta}_{0}\right) \cdot \boldsymbol{r}}$ represents a valley dependent shift of momentum. After this gauge transformation, the new Hamiltonian $\hat{H}^{\prime}$ can be written solely in terms of the $\breve{b}$ harmonics,

$$
\begin{aligned}
\hat{H}^{\prime} & =v\left(\hat{\boldsymbol{p}}+\frac{1}{6}\left[3 \boldsymbol{b}_{0}+\tau_{3}\left(\boldsymbol{b}_{4}+\boldsymbol{b}_{5}\right)\right]\right) \cdot \boldsymbol{\sigma} \\
& +U_{E^{\prime}} v b\left(\tau_{1} \operatorname{Re} f^{\prime}-\tau_{2} \operatorname{Im} f^{\prime}\right) \sigma_{3}+U_{G} v\left(\tau_{1} \operatorname{Re} \boldsymbol{g}^{\prime}-\tau_{2} \operatorname{Im} \boldsymbol{g}^{\prime}\right) \boldsymbol{\sigma} \\
& +U_{G^{\prime}} v\left(\tau_{1} \operatorname{Re}\left[\hat{R}_{\frac{\pi}{2}} \boldsymbol{g}^{\prime}\right]-\tau_{2} \operatorname{Im}\left[\hat{R}_{\frac{\pi}{2}} \boldsymbol{g}^{\prime}\right]\right) \boldsymbol{\sigma} \\
f^{\prime} & =\frac{2}{\sqrt{3}}\left(1+e^{i \boldsymbol{b}_{1} \cdot \boldsymbol{r}}+e^{i \boldsymbol{b}_{2} \cdot \boldsymbol{r}}\right), \boldsymbol{g}^{\prime}=\frac{2 i}{\sqrt{3}}\left(\boldsymbol{b}_{0}+\boldsymbol{b}_{2} e^{i \boldsymbol{b}_{1} \cdot \boldsymbol{r}}+\boldsymbol{b}_{4} e^{i \boldsymbol{b}_{2} \cdot \boldsymbol{r}}\right) .
\end{aligned}
$$

\subsection{Characteristic Miniband Spectra}

Characteristic miniband spectra, calculated by numerical diagonalisation in the basis of zone-folded plane waves of $K_{+}$and $K_{-}$Dirac electrons, are shown in Fig. 7.2(b,c). The choices of phenomenological parameters used to calculate these spectra, marked with black dots in the lower right panel of Fig. 7.3, correspond to the direction in the parameter space set by Eq. (7.2) with $\theta=0$. Since nesting obscures some of the dispersion branches, it is useful to plot them over both the smaller mBZ (left) and the larger mBZ (middle). Also, we note that the calculated spectra will be electron-hole asymmetric, $\epsilon(\boldsymbol{k}) \neq-\epsilon(\boldsymbol{k})$, unless either $U_{E}=0$ or $U_{G}=U_{G^{\prime}}=0$.

Generically, we find either a gapped edge of the first moiré miniband (on the conduction and/or valence band side of the graphene spectra) for a strong moiré perturbation, or gapless spectra with overlapping minibands for a weak moiré perturbation. In all cases, the main Dirac point is preserved with a renormalized Dirac velocity, $\left(1-12 U_{E^{\prime}}^{2}-24 U_{G}^{2}\right) v$. The parameter range where the spectrum 
a) $U_{E^{\prime}}=0, U_{G}=0, U_{G^{\prime}}=0$
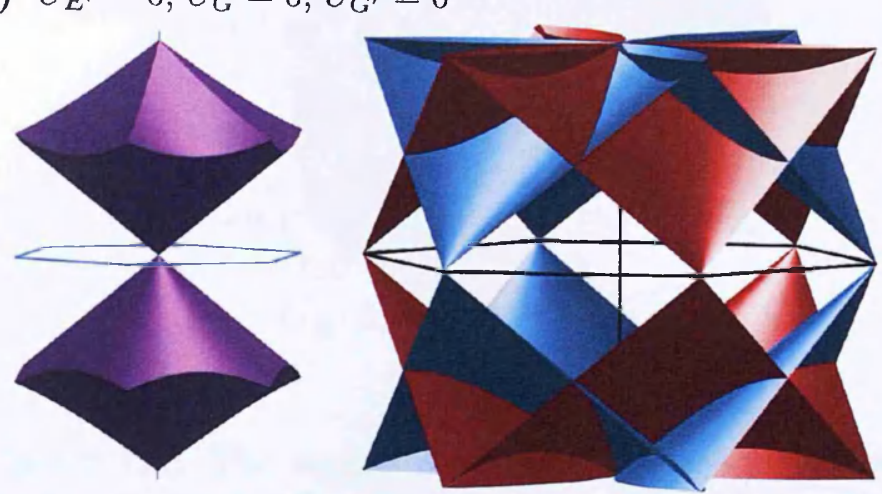

b) $U_{E^{\prime}}=0.02, U_{G}=-0.04, U_{G^{\prime}}=0$
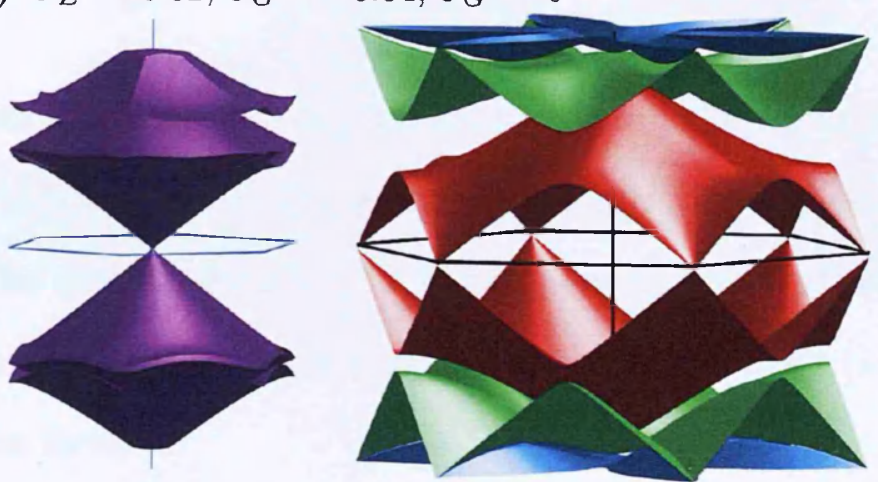

c) $U_{E^{\prime}}=0.07, U_{G}=-0.14, U_{G^{\prime}}=0$
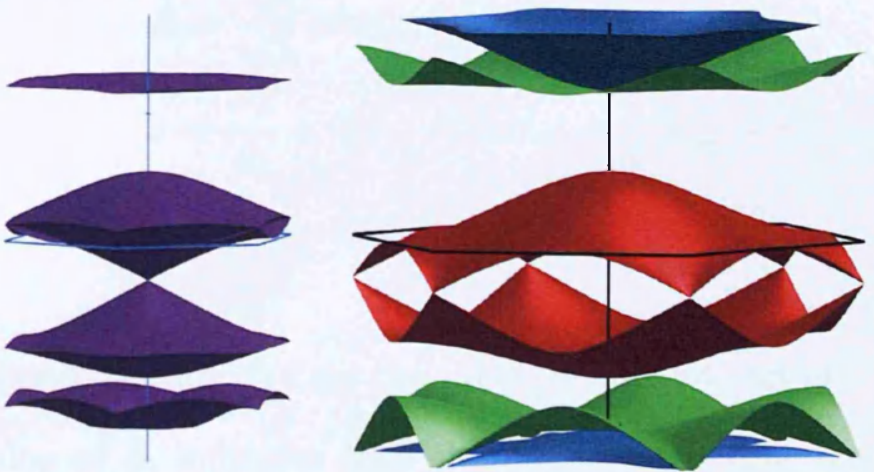
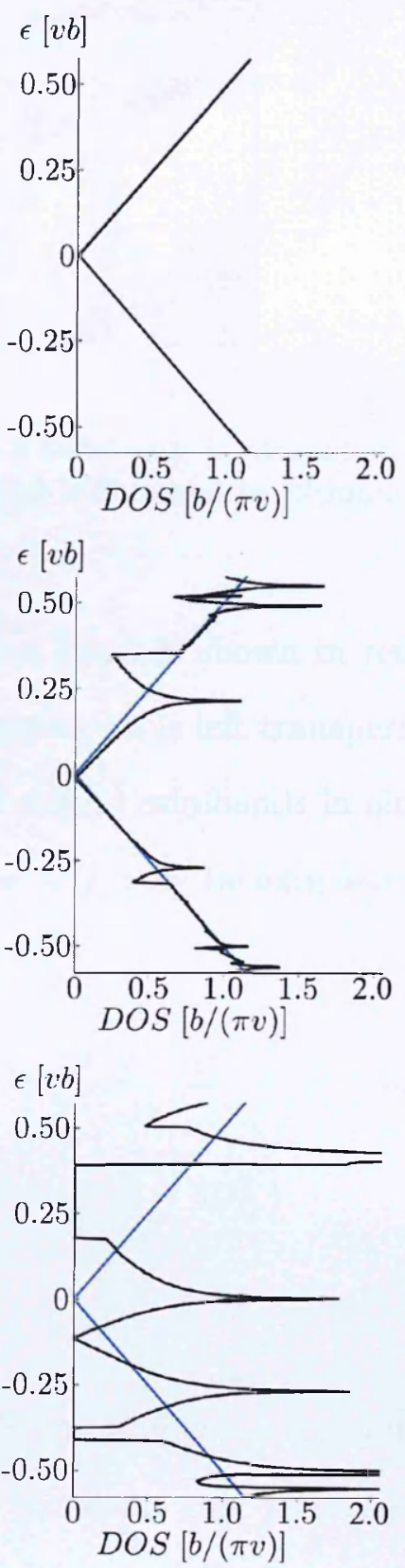

Figure 7.2: Numerically calculated moiré minibands shown in the smaller mBZ (left) and larger $\mathrm{mBZ}$ (centre), and the corresponding density of states (right). A Van Hove singularity, originating from the first moiré miniband (in both the conduction and valence bands) is always present for the perturbed spectra. 

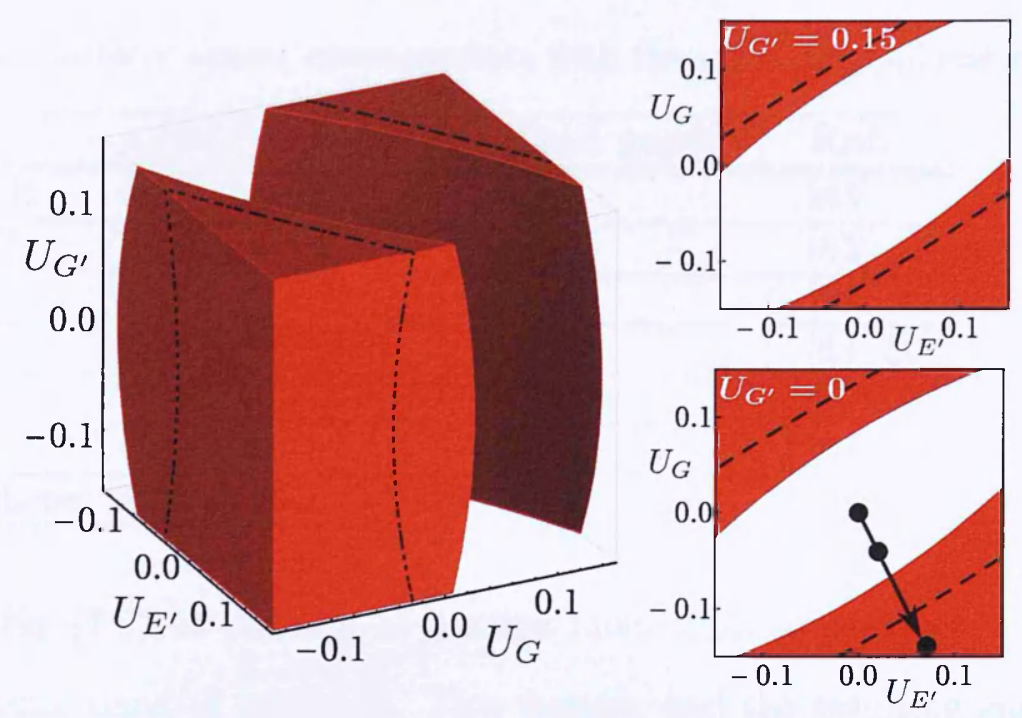

Figure 7.3: The regions of parameter space for which a band gap is present in the conduction band. The parameter space for the valence band is obtained by flipping the sign of $U_{E^{\prime}}$.

has a gap at the first miniband edge in the conduction band is shown in red in Fig. 7.3, whereas the parameter range with a gapless spectrum is left transparent. The magnitude of the band gap between the first and second minibands in either the conduction band $(s=1)$ or the valence band $(s=-1)$, may be expressed in the form

$$
\begin{aligned}
& \Delta=\frac{v b}{\sqrt{3}} \min (c, d) \\
& c \approx \frac{-1}{2}+\left|4 U_{E^{\prime}}-6 s U_{G}\right|+\frac{4}{3}\left(U_{E^{\prime}}^{2}+6 s U_{E^{\prime}} U_{G}-2 U_{G}^{2}-3 U_{G^{\prime}}^{2}\right) \\
& d \approx\left|U_{E^{\prime}}-2 s U_{G}\right|+\frac{3}{2}\left(3 U_{E^{\prime}}^{2}-4 U_{G^{\prime}}^{2}\right) .
\end{aligned}
$$

where $\frac{v b}{\sqrt{3}} c$ and $\frac{v b}{\sqrt{3}} d$ are the values of the indirect and direct band gaps. A negative value of $\Delta$ indicates that the bands are overlapping (no band gap, transparent volume of Fig. 7.3). A more detailed discussion is found in appendix B.

\subsection{Conclusion}

To summarize, Dirac electrons in graphene heterostructures with hexagonal crystals with a three times larger unit cell than that of graphene are likely to have a 
Table 7.1: Surfaces almost commensurate with the $\sqrt{3} \times \sqrt{3}$ graphene superlattice.

\begin{tabular}{lllll}
\hline Surface & $a_{s}[\AA]$ & $E_{1}(\theta=0)[\mathrm{eV}]$ & Band gap $[\mathrm{eV}]$ & Ref. \\
\hline \hline $\mathrm{InAs}(111) \mathrm{B}$ & 4.28 & 0.026 & 0.35 & {$[83]$} \\
\hline $\mathrm{InP}(111) \mathrm{B}$ & 4.15 & 0.145 & 1.34 & {$[83]$} \\
\hline $\mathrm{PdTe}_{2}$ & 4.04 & 0.289 & 0.2 & {$[84,85]$} \\
\hline $\mathrm{PtTe}_{2}$ & 4.03 & 0.303 & 0.8 & {$[84,86]$} \\
\hline $\mathrm{InSe}$ & 4.05 & 0.276 & $\approx 1$ & {$[87]$} \\
\hline $\mathrm{h}-\mathrm{GaTe}$ & 4.04 & 0.289 & 2.1 & {$[88-90]$} \\
\hline$\sqrt{3} a$ graphene & 4.26 & n/a & 0 & {$[28]$} \\
\hline
\end{tabular}

band gap, Eq. (7.5), at the edge of the first moiré miniband, either in the conduction or valence band of graphene. This feature, and the resulting suppression of the electron density of states in graphene, take place at the energy $\epsilon_{\theta} \sim \pm v b / \sqrt{3}$, counted from the ungapped Dirac point. The energy scale where such feature occurs depends on the difference between the lattice constants of the two crystals and their misalignment.

For each hexagonal crystal, the lowest possible value of $\epsilon_{0}$ is shown in Table 7.1: it corresponds to the perfect alignment of the two lattices, $\theta=0$, and it is set by the lattice mismatch $\delta$. In this table, we list several semiconductors which can provide facets nearly commensurate with the $\sqrt{3} \times \sqrt{3}$ Kekule superlattice in graphene. Two of them are zinc blende type crystals, InAs and InP, whose (111)B surfaces retain the hexagonal structure of the top layer of As or $\mathrm{P}$ atoms without surface reconstructions [91-93]. Of these two, $\operatorname{InAs}(111) \mathrm{B}$ has a work function [94] close to that of free-standing graphene [95], and a polar surface which causes a downward band bending, sometimes leading to an accumulation electron layer near the surface. However, it is possible to produce accumulation-free $\operatorname{InAs}(111) \mathrm{B}$ surface [96, 97], or deplete graphene-InAs using gate-controlled doping in a field effect transistor. Due to a smaller size of the energy $\epsilon_{0}$ than the InAs band gap (see Table 7.1) it should be possible to reach the gap at the first miniband edge in graphene, before depleting states in the valence band of the InAs substrate, thus producing a graphene-based field effect transistor with improved current on/off ratio. Since the energy of the first moiré miniband edge depends on the misalignment 
angle, the proposed device will work only for a limited range of (small) misalignment angles. By comparing the size of the corresponding values of $\epsilon_{0}$ to the band gaps of materials Table 7.1, we suggest that $\operatorname{InP}(111) \mathrm{B}, \mathrm{hGaTe}$ and InSe may also be suitable for producing high on/off current ratio in field-effect transistors, but for $\mathrm{PdTe}_{2}$ and $\mathrm{PtTe}_{2}$ the band gap is too small as compared to $\epsilon_{0}$. 


\section{Chapter 8}

\section{Raman Scattering by Phonons in}

\section{Graphene}

\subsection{Introduction}

The electron-phonon interaction (EPI) determines many kinetic properties of metals and semiconductors. Its measurement, together with the characterization of the spectra of lattice vibrations, represents an important step in the study of new materials. Graphene [6, 99], a two-dimensional sheet of carbon atoms arranged on a honeycomb lattice, is no exception. Over the several years since its discovery, the phonon spectra and coupling between Dirac-type electrons and phonons in graphene have been studied both theoretically $[27,39,100-108]$ and experimentally [109-115], in particular, using inelastic (Raman) scattering of light. Using the irreducible representations of the symmetry group of the honeycomb crystal of monolayer graphene (section 1.5) it has been established that the lattice modes engaged in EPI include in-plane optical phonons in the centre of the hexagonal Brillouin zone (BZ), transverse optical $\left(\mathrm{TO}_{\mathrm{K}_{ \pm}}\right)$as well as longitudinal optical $\left(\mathrm{LO}_{\mathrm{K}_{ \pm}}\right)$ and longitudinal acoustic $\left(\mathrm{LA}_{\mathrm{K}_{ \pm}}\right)$phonons in the vicinity of the BZ corners $\mathrm{K}_{+}$ and $\mathrm{K}_{-} .^{*}$

${ }^{*}$ Time reversal symmetry forbids electronic coupling to $\mathrm{TA}_{\mathrm{K}_{ \pm}}$. This mode belongs to irreducible representation $E^{\prime \prime}$ of $c_{6 v^{\prime \prime}}$, and therefore couples with the $\tau_{x}, \tau_{y}$ electronic matrices. These are odd under time reversal. Also, out-of-plane modes can only couple quadratically, since the 

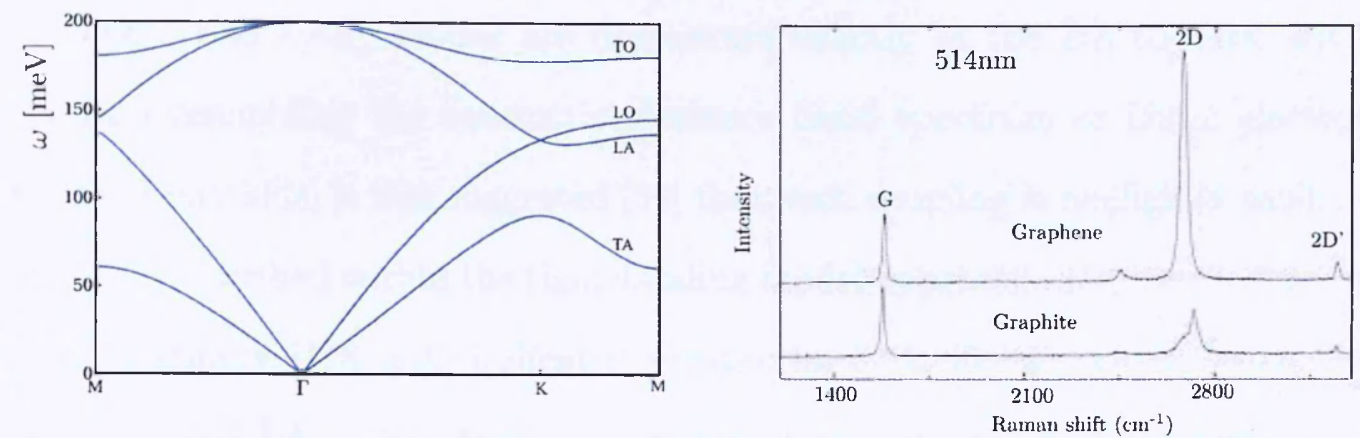

Figure 8.1: The in-plane phonon modes (left panel) calculated using a simplistic two parameter valence-force field model, separately accounting for the forces required to stretch the carbon-carbon bond and change the bond angle. The Raman spectra of graphene and graphite (right panel), measured experimentally using $514 \mathrm{~nm}$ light [taken from Ref. [98]]. For comparison $1 \mathrm{meV}=8.06554 \mathrm{~cm}^{-1}$.

By now, $\Gamma$-point optical phonons $\left(\mathrm{LO}_{\Gamma}\right.$ and $\left.\mathrm{TO}_{\Gamma}\right)$ and transverse optical $\left(\mathrm{TO}_{\mathrm{K}_{+}}\right)$ phonons in the corner of the hexagonal Brillouin zone (BZ) have been extensively studied using Raman and X-ray spectroscopy (see Fig. 8.1). Their coupling to electrons has been calculated using ab-initio density functional theory (DFT) $[100,102]$, incorporated in the graphene tight-binding model [101], and subjected to the study of renormalisation by the electron-electron interaction [107]. Based on the theory of phonon renormalisation by the EPI [100-103], the experimentallyobserved variation of the G-line in Raman (attributed to the excitation of one $\Gamma$-point optical phonon) as a function of carrier density in graphene [113] and a fine structure it acquires at a strong magnetic field under the condition of magnetophonon resonance $[104,105,116]$ have permitted a direct measurement of the EPI constant with this mode. A further comparison between the integral intensities of the two-phonon peaks in Raman attributed to the excitation of a pair of $\Gamma$-point optical phonons (2D' peak) and a pair of $\mathrm{TO}_{\mathrm{K}_{ \pm}}$phonons (2D peak) facilitated the experimental determination of the corresponding EPI constants.

In contrast to the above-discussed phonon branches, the Raman signature of the $\mathrm{L} \mathrm{O}_{\mathrm{K}_{ \pm}}$and $\mathrm{LA}_{\mathrm{K}_{ \pm}}$modes in graphene and their interaction with electrons have not been satisfactorily established. This pair of lattice excitations is quite interesting. system assumed to be symmetric under reflection in the graphene plane (the substrate neglected until section 8.5). These modes will not be considered further. 
The $\mathrm{LO}_{\mathrm{K}_{ \pm}}$and $\mathrm{LA}_{\mathrm{K}_{ \pm}}$modes are degenerate exactly at the $\mathrm{BZ}$ corners, with a spectrum resembling the conduction/valence band spectrum of Dirac electrons. On the theory side, it was suggested [39] that such coupling is negligibly weak and cannot be described within the tight-binding model approach, despite the fact that $a b$-initio studies $[118,119]$ indicated substantial EPI effects. At the same time, the $\mathrm{LO}_{\mathrm{K}_{ \pm}}$and $\mathrm{LA}_{\mathrm{K}_{ \pm}}$double-phonon Raman lines have not been unambiguously identified in the published Raman data.

In this chapter, we use a analytical description of the pair of longitudinal phonons in the vicinity of the BZ corners to show that the interaction between Dirac electrons in graphene and these modes can be described within a minimal tight-binding model and estimate the corresponding coupling using DFT, and calculate the lineshape of the corresponding two-phonon signal in Raman. We predict that the latter is strongly asymmetric, reflecting a substantial trigonal asymmetry of these phonons' dispersion around the BZ corners, with the linewidth being dependent on the energy of the incoming photon. We also show that the presence of a superlattice perturbation modifies the EPI, resulting in a non-isotropic renormalisation of the energy of the $\mathrm{TO}_{\mathrm{K}_{ \pm}}$mode.

\subsection{In-plane Phonon Modes}

The relevant phonon branches include optical phonons near the BZ center where we describe the phonon state using the full value of wave vector $\mathbf{q}$ and in the vicinity of the $\mathrm{BZ}$ corner where $\mathrm{q} \equiv q(\cos \varphi, \sin \varphi)$ is the valley wave vector part of the total wave vector $\boldsymbol{K}_{+}+$q. A full microscopic theoretical description of the phonon spectrum $[27,103,120,121]$ should be done by means of diagonalization of a full $4 \times 4$ dynamical matrix $\mathbb{D}_{4 \times 4}$ determined in the space of the inplane (xy) displacements of $\mathrm{A}$ and $\mathrm{B}$ sublattice atoms: $A_{x}, A_{y}, B_{x}, B_{y}$. For the sake of simplicity of the Raman spectrum analysis, we perform a partial diagonalization of the actual dynamical matrix $\mathbb{D}_{4 \times 4}$ and employ a Schrieffer-Wolff transformation [122] to reduce it to a block-diagonal form describing each of the phonon branches 
around the $K\left(K^{\prime}\right)$ points separately.

A degenerate pair of $\mathrm{TO}$ and $\mathrm{LO}$ modes at the center of the $\mathrm{BZ}\left(q \ll a_{c c}^{-1}\right.$, where $a_{c c}$ is the A-B bond length), with polarization $\left|v_{\mathrm{LO}}\right\rangle_{\Gamma}=(1 / 2 q)\left(q_{x}, q_{y},-q_{x},-q_{y},\right)$ and $\left|v_{\mathrm{TO}}\right\rangle_{\Gamma}=\frac{1}{2 q}\left(q_{y},-q_{x},-q_{y}, q_{x},\right)$ have an almost isotropic dispersion [123], $\omega_{\mathrm{LO}-\mathrm{TO}}^{2}(\Gamma, \mathbf{q})$ $\omega_{\Gamma}^{2}+\lambda_{\Gamma} q^{2}\left(\omega_{\Gamma} \approx 0.2 \mathrm{eV}\right)$. In the vicinity of the $\mathrm{K}_{+}$-point, the highest energy branch (TO) is only slightly anisotropic with respect to the direction of the phonon wave vector $\mathbf{q}$ in the $\mathrm{K}_{\zeta}$ valley $(\zeta= \pm 1)$ [polarization $\left|v_{\mathrm{TO}}\right\rangle_{\boldsymbol{K}_{+}}=\frac{1}{2}(i,-1, i, 1)$ and $\left.\left|v_{\mathrm{TO}}\right\rangle_{K_{-}}=\left|v_{\mathrm{TO}}\right\rangle_{K_{+}}^{*}\right]: \omega_{\mathrm{TO}}^{2}(\mathbf{q}) \simeq \omega_{\mathrm{TO}}^{2}+\lambda_{\mathrm{TO}} q^{2}+\zeta \chi_{\mathrm{TO}} q^{3} \cos (3 \varphi)$, where $\omega_{\mathrm{TO}} \approx 0.15 \mathrm{eV}[114]$.

This contrasts with the much stronger anisotropy of the pair of LO-LA phonons, which are degenerate in the BZ corners K and K', where both have the same energy $\omega_{\mathrm{L}} \approx 0.15 \mathrm{eV}$ [114], and polarization vectors $\left|v_{1}\right\rangle_{\boldsymbol{K}_{+}}=2^{-1 / 2}(i, 1,0,0),\left|v_{2}\right\rangle_{\boldsymbol{K}_{+}}=$ $2^{-1 / 2}(0,0,-i, 1),\left|v_{1}\right\rangle_{\boldsymbol{K}_{-}}=\left|v_{1}\right\rangle_{\boldsymbol{K}_{+}}^{*}$, and $\left|v_{2}\right\rangle_{\boldsymbol{K}_{-}}=\left|v_{2}\right\rangle_{\boldsymbol{K}_{+}}^{*}$ form the 4-dimensional irreducible representation $\mathrm{G}$ of the symmetry group of the crystal. For each valley, the pair $\left[\left|v_{1}\right\rangle,\left|v_{2}\right\rangle\right]$ can be used as a basis to describe the polarization of lattice displacements at a finite valley wave vector $\mathbf{q}$, as $|v\rangle=\eta_{1}\left|v_{1}\right\rangle+\eta_{2}\left|v_{2}\right\rangle$. Then, the LO-LA spectrum can be described [121] using the $2 \times 2$ dynamical matrix acting in the space of two-component vectors $\eta=\left(\eta_{1}, \eta_{2}\right)$

$$
\begin{aligned}
\mathbb{D}_{K_{+}}(\mathbf{q}) & =\left(\begin{array}{cc}
\omega_{\mathrm{L}}^{2}+\lambda_{\mathrm{L}} q^{2} & \rho_{\mathrm{L}}\left(\kappa^{*}\right)^{2}-s_{\mathrm{L}} \kappa \\
\rho_{\mathrm{L}} \kappa^{2}-s_{\mathrm{L}} \kappa^{*} & \omega_{\mathrm{L}}^{2}+\lambda_{\mathrm{L}} q^{2}
\end{array}\right), \\
\mathbb{D} \eta & =\omega^{2} \eta, \quad \kappa \equiv q_{x}+i q_{y}=q e^{i \varphi}, \\
\mathbb{D}_{K_{-}}(\mathbf{q}) & =\mathbb{D}_{K_{+}}^{*}(-\mathbf{q}), \quad \eta_{\boldsymbol{K}_{-}}(\mathbf{q})=\eta_{\boldsymbol{K}_{+}}^{*}(-\mathbf{q}) .
\end{aligned}
$$

In group-theory terms, this representation is equivalent to the irreducible representation provided by the two-component electronic wave functions in the valleys $\mathrm{K}_{+}$and $\mathrm{K}_{-}$of monolayer and bilayer graphene. The dynamical matrix (8.1) has structure similar to that of the electronic Hamiltonian [124], with $s_{\mathrm{L}}$ playing the role of 'Dirac velocity' and the term with $\rho_{\mathrm{L}}$ that of the 'warping term'. On the basis of diagonalization of the full $4 \times 4$ dynamical matrix, we observe that $s_{L}>0$, 
$\rho_{L}>0$. The corresponding spectrum and polarization of the $\mathrm{LO}(+)$ and $\mathrm{LA}(-)$ modes near $\mathrm{K}$ and $\mathrm{K}$ ' read

$$
\begin{aligned}
\omega_{\boldsymbol{K}_{+} \pm}^{2}(\mathbf{q}) & \simeq \omega_{\mathrm{L}}^{2}+\lambda_{\mathrm{L}} q^{2} \pm \Pi^{1 / 2}, \omega_{\boldsymbol{K}_{+}^{\prime} \pm}(\mathbf{q})=\omega_{\boldsymbol{K}_{+} \pm}(-\mathbf{q}) \\
\Pi & =s_{\mathrm{L}}^{2} q^{2}+\rho_{\mathrm{L}}^{2} q^{4}-2 \rho_{\mathrm{L}} s_{\mathrm{L}} \operatorname{Re} \kappa^{3} \\
\eta_{\boldsymbol{K}_{+} \pm}(\mathbf{q}) & =\frac{1}{\sqrt{2 \Pi}}\left(\begin{array}{c} 
\pm\left[\rho_{\mathrm{L}}\left(\kappa^{*}\right)^{2}-s_{\mathrm{L}} \kappa\right] \\
\sqrt{\Pi}
\end{array}\right) .
\end{aligned}
$$

\subsection{Electron-Phonon Coupling}

Below, we use the electronic tight-binding model to describe the electron coupling with all of the above-mentioned lattice modes. The minimal tight-binding model reads

$$
H=\sum_{i} \epsilon_{i} c_{i}^{\dagger} c_{i}-\sum_{\langle i, j\rangle}\left[t_{i j} c_{i}^{\dagger} c_{j}+\text { h.c. }\right]
$$

Here the first term takes into account the fact that the on-site energy $\epsilon_{i}$ on the $i$ th site on the honeycomb lattice may depend on the mutual distance between neighboring $(\mathrm{A} / \mathrm{B})$ carbon atoms. The second term describes nearest-neighbor (A/B) hopping. For an unperturbed honeycomb lattice, $\epsilon_{i}=\epsilon_{0}$ and $t_{i j}=t$, and electrons with momenta $\boldsymbol{K}_{+}+\mathbf{p}\left(\boldsymbol{K}_{-}+\mathbf{p}\right)$ in the vicinity of the BZ corners $[\mathbf{p}=p(\cos \phi, \sin \phi)$ is the valley momentum] are described by the Hamiltonian

$$
H_{\mathrm{e}}=\psi_{\mathbf{p}}^{\dagger}\left(\begin{array}{cc}
\epsilon_{0}+v \mathbf{p} \boldsymbol{\sigma} & 0 \\
0 & \epsilon_{0}+v \mathbf{p} \boldsymbol{\sigma}
\end{array}\right) \psi_{\mathbf{p}}
$$

where $\psi^{\dagger}=\left(\psi_{A, \boldsymbol{K}_{+}}^{\dagger}, \psi_{B, \boldsymbol{K}_{+}}^{\dagger}, \psi_{B, K_{-}}^{\dagger},-\psi_{A, \boldsymbol{K}_{-}}^{\dagger}\right), \sigma_{x, y}$ are Pauli matrices in the space of the electron amplitude on A and B sublattices, and $v=3 a_{c c} t / 2 \hbar$.

Modulation of the hopping energy $t_{i j}$ is known to lead to EPI with $\Gamma$-point optical phonons and $\mathrm{TO}_{\mathrm{K}_{ \pm}}$phonons [39]. This can be established using an expansion, $t_{i j}=t+t^{\prime}\left(\mathbf{u}_{j}-\mathbf{u}_{i}\right) \mathbf{e}_{i j}$, of the hopping parameter in the lattice displacements $\mathbf{u}_{i}$. Here, $t^{\prime}=\partial t / \partial a_{c c}$ is the hopping parameter derivative with respect to the AB 
bond length $a_{c c}$, and $\mathbf{e}_{i j}$ is a unit vector connecting two neighboring sites $i$ and $j$. Similarly, the dependence of the on-site energy $\epsilon_{i}$ with respect to the surrounding three honeycomb lattice sites $j$,

$$
\epsilon_{i}=\epsilon_{0}+\frac{\epsilon^{\prime}}{3} \sum_{j}\left(\mathbf{u}_{j}-\mathbf{u}_{i}\right) \mathbf{e}_{i j}
$$

leads to EPI parameterized using $\epsilon^{\prime}=\partial \epsilon_{0} / \partial a_{c c}$. To compare the two EPI parameters, we employed ${ }^{\dagger}$ the DFT-based Vienna ab initio simulation package (VASP) [125] with a plane-wave basis set used within the framework of the projector augmented-wave method to obtain first principles values for the derivative of the on-site energy with respect to the carbon-carbon bond length. The plane-wave cutoff energy was $500 \mathrm{eV}$, which corresponds to a very large basis set where basis set superposition errors are negligible, and a $72 \times 72$ Monkhorst-Pack grid was used in $k$-space. Results were obtained using three different DFT functionals: the Ceperley-Alder local density approximation functional (LDA), the PerdewBurke-Ernzerhof generalized gradient approximation functional (PBE), and the Heyd-Scuseria-Ernzerhof screened hybrid functional (HSE06). The derivative of the on-site energy was calculated by numerical differentiation using a $0.01 \AA$ displacement in the bond length preserving lattice symmetry. Since the on-site energy in the tight-binding model sets the Fermi energy relative to the vacuum, the key to obtaining first principles results for the on-site energy $\epsilon_{0}\left(a_{c c}\right)$ is to obtain the correct reference energy of the vacuum in each step of the calculation. This was done by computing the electrostatic potential in real space, which saturates at a 'zero level' far from the graphene layer. This method was previously used to compare absolute values of the Fermi energy of carbon nanotubes of varying diameter [126]. Our results for parameters $\epsilon^{\prime}$ and $t^{\prime}$ are summarized in Table 1, in comparison with earlier calculated $t^{\prime}$ values.

Substituting the expansion of $t_{i j}$ and $\epsilon_{i}$ into the tight-binding model (8.3) yields electron coupling to all of the above-discussed phonons in the centre $(\nu=$

\footnotetext{
†This part of the work was carried out by V. Zóloymi, who also compiled table 8.1
} 
Table 8.1: The DFT values of parameters $\epsilon^{\prime}$ and $t^{\prime}$ in units of $e V / \AA$ (calculated here and taken from recent literature).

\begin{tabular}{llllll}
\hline \hline & LDA & PBE & HSE06 & $\overline{\text { SSH }}$ & DFPT \\
\hline$-\epsilon^{\prime}$ & 6.1 & 6.0 & 6.3 & & \\
$-t^{\prime}$ & 4.5 & 4.5 & 5.1 & $3.4[127] ; 5.3[128] ; 5.5[129]$ & $7.8[100,130]$ \\
\hline \hline
\end{tabular}

$\left.\mathrm{LO}_{\Gamma}, \mathrm{TO}_{\Gamma}\right)$ and corners $\left(\nu=\mathrm{TO}_{\mathrm{K}_{ \pm}}, \mathrm{LO}_{\mathrm{K}_{ \pm}}, \mathrm{LA}_{\mathrm{K}_{ \pm}}\right)$of the $\mathrm{BZ}$,

$$
\begin{gathered}
H_{\mathrm{EPI}}=\psi_{\mathbf{p}-\mathbf{q}}^{\dagger}\left[h_{\mathrm{L}}+h_{\mathrm{TO}}+h_{\Gamma}\right] \psi_{\mathbf{p}}+\text { h.c. } ; \\
h_{\mathrm{L}}=\frac{g_{\mathrm{L}}}{\sqrt{N}} \sum_{\nu}\left(\begin{array}{cc}
0 & \Lambda_{\mathbf{q}, \nu} \alpha_{\boldsymbol{K}_{+}+\mathbf{q}, \nu}^{\dagger} \\
\Lambda_{-\mathbf{q}, \nu}^{\dagger} \alpha_{\boldsymbol{K}_{-}+\mathbf{q}, \nu}^{\dagger} & 0
\end{array}\right), \quad \Lambda_{\mathbf{q}, \nu}=\left(\begin{array}{cc}
0 & \eta_{2, \mathbf{K} \nu}^{*}(\mathbf{q}) \\
\eta_{1, \mathbf{K} \nu}^{*}(\mathbf{q}) & 0
\end{array}\right) ; \\
h_{\Gamma}=\frac{g_{\Gamma}}{\sqrt{N}} \sum_{\nu=\mathrm{LO}, \mathrm{TO}}\left(\begin{array}{cc}
\mathbf{l}_{\nu} \boldsymbol{\sigma} & 0 \\
0 & -\mathbf{l}_{\mathbf{q}, \nu} \boldsymbol{\sigma}
\end{array}\right) \\
b_{-\mathbf{q}, \nu}^{\dagger}, \\
h_{\mathrm{TO}}=\frac{g_{\mathrm{TO}}}{\sqrt{N}}\left(\begin{array}{cc}
0 & \beta_{\mathrm{LO}}^{\dagger} \\
\boldsymbol{K}_{+}+\mathbf{q} & \sigma_{z} \\
\beta_{\boldsymbol{K}_{-}+\mathbf{q}} \sigma_{z} & 0
\end{array}\right) ;
\end{gathered}
$$

where $N$ is the number of unit cells and the coupling constants are written using the carbon mass $m$,

$$
g_{\mathrm{L}}=\frac{-\epsilon^{\prime} \sqrt{\hbar}}{\sqrt{4 m \omega_{\mathrm{L}}}}, \quad g_{T O}=\frac{3 t^{\prime} \sqrt{\hbar}}{\sqrt{2 m \omega_{\mathrm{TO}}}}, \quad g_{\Gamma}=\frac{3 t^{\prime} \sqrt{\hbar}}{\sqrt{4 m \omega_{\Gamma}}}
$$

Here, $\alpha^{\dagger}, \beta^{\dagger}, b^{\dagger}$ are creation operators of the corresponding LO/LA, TO and $\Gamma$ point optical phonons, whereas all phonon annihilation operators are incorporated in the Hermitian conjugated (h.c.) terms. In $H_{\mathrm{EPI}}$, the lower-left blocks describe the emission of a phonon due to intervalley scattering of an electron from $\mathrm{K}_{+}$to $\mathrm{K}_{-}$valley, and the upper-right blocks - scattering from $\mathrm{K}_{-}$to $K_{+}$valley. The form of $H_{\mathrm{L}}$ in $H_{\mathrm{EPI}}$ agrees with that proposed using symmetry-based phenomenology in Ref. [39], however, the comparison of the DFT-calculated coupling parameters, Table 1, suggests that the coupling of electrons to the LA/LO branches is not diminishingly small, in contrast to Refs. [39, 100]. 

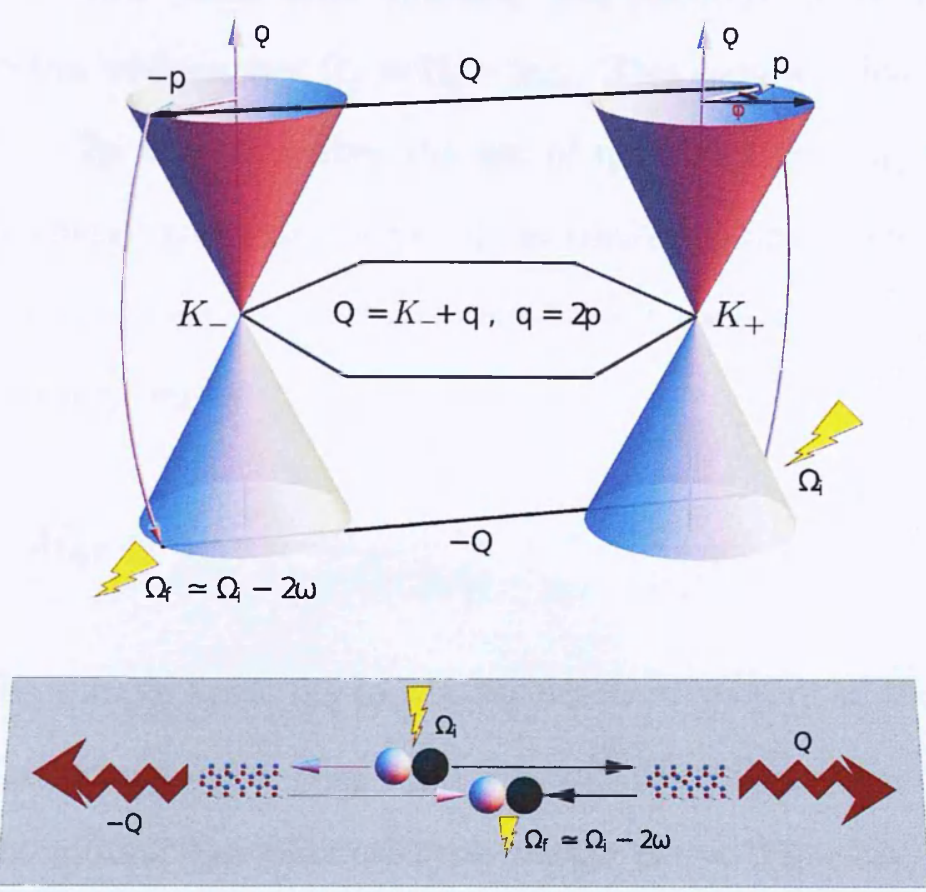

Figure 8.2: Resonant two-phonon scattering in graphene. The cones indicate the electronic bands in the vicinity of the Brillouin zone corners, labeled $K_{+}$and $K_{-}$. Lightning marks indicate an incoming (outgoing) photon. The electron-hole pair generated in one valley scatters to the other valley (by emission of phonons with wave vectors $\pm \mathbf{Q}$ ), where it recombines to contribute to the Raman signal.

\subsection{LO-LA Raman Spectra}

Finally, we can use EPI in Eq. (8.4) to calculate the spectral density of inelastic light scattering accompanied by the emission of a pair of $\mathrm{LA}_{\mathrm{K}_{ \pm}}$or $\mathrm{LO}_{\mathrm{K}_{ \pm}}$phonons. The two-phonon Raman scattering is a fully-resonant process. It is illustrated in Fig. 8.2, and was discussed in detail in relation to the Raman spectroscopy of transverse phonons [39]. An incoming photon, with energy $\Omega_{i}$, generates an electron-hole pair in the vicinity of one of the valleys (the $\mathrm{K}_{+}$-point in the upper panel of Fig. 8.2). Owing to energy and momentum conservation and the negligible momentum of the incoming photon, the photoexcited electron has the same momentum, $\mathbf{p}=\left(\Omega_{i} / 2 v\right)(\cos \phi, \sin \phi)$ as it had in the emptied state (which can be viewed as a valence band hole). Then, both the electron and hole scatter to the other valley (the $\mathrm{K}_{-}$-point in Fig. 8.2) emitting a pair of phonons with opposite wave vectors $\pm \mathrm{Q}$. The kinetics of this process are such that both electron 
and hole retrace their paths after emitting $\pm \mathbf{Q}$ phonons, to be able to recombine into a photon with energy $\Omega_{f}=\Omega_{i}-2 \omega_{\nu}$. This requires that $\mathbf{Q}=\mathbf{K}_{-}+2 \mathbf{p}$ and $-\mathbf{Q}=\mathbf{K}_{+}-2 \mathbf{p}$ and prescribes the use of $\mathbf{q}=-2 \mathbf{p}\left(q \sim q_{0} \approx \Omega_{i} / v\right)$ when calculating the energy and polarization of the corresponding modes.

Similarly to TO phonons $[39,106]$, the matrix element of the LO-LA fully resonant process is given by

$$
M_{\mathbf{q}, \nu}=\frac{\sqrt{6 \pi^{3}} e^{2} L^{2} g_{L}^{2}}{8 N \sqrt{\Omega_{i}+\Omega_{f}}} \frac{\left[\mathbf{e}_{\mathbf{q}} \times \mathbf{e}_{i}\right]_{z}\left[\mathbf{e}_{\mathbf{q}} \times \mathbf{e}_{f}^{*}\right]_{z}}{\left[v\left(q-q_{0}\right)-2 i \gamma\right]^{3 / 2}} A_{\nu}(\mathbf{q})
$$

where $L^{2}$ is the sample area, $\mathbf{e}_{i}, \mathbf{e}_{f}$ are polarization vectors of the photons and $\mathbf{e}_{\mathbf{q}}=\mathbf{q} / q$. The denominator $\left[v\left(q-q_{0}\right)-2 i \gamma\right]^{3 / 2}$ describes a sharp peak of the matrix element around the phonon wave vector $q_{0}=\left(\Omega_{i}+\Omega_{f}\right) /(2 v) \approx \Omega_{i} / v$ corresponding to resonant backscattering. The width $\delta q \sim \gamma / v$ of this peak is determined by the electron inelastic-scattering rate $2 \gamma$. The factor

$$
A_{\mathrm{LO}, \mathrm{LA}}(\mathbf{q})=-\frac{1}{\sqrt{6 \pi}}\left[1 \pm \frac{\rho_{\mathrm{L}} q^{2}-s_{\mathrm{L}} q \cos 3 \varphi}{\Pi^{1 / 2}}\right]
$$

(+ stands for the LA and - for the LO mode) shows that the kinetics of the LO-LA fully resonant process are strongly affected by the anisotropy of their dispersion, Eq. (8.2), which increases upon departure from the BZ corners. This anisotropy, shown in Fig. 8.3, will be observable in the spectral density of the LO-LA signal,

$$
\mathcal{L}(\omega)=\frac{4 \Omega_{f}^{2}}{\pi c^{4}} \sum_{\nu=\mathrm{LO}, \mathrm{LA}} \int \frac{d^{2} q}{(2 \pi)^{2}}\left|M_{\mathbf{q}, \nu}\right|^{2} \delta\left(\omega-2 \omega_{\nu}(\mathbf{q})\right)
$$

because the wave vector of the emitted phonons $q \equiv q_{0} \approx \Omega_{i} / v$ may be tuned by changing the photon energy $\Omega_{i}$.

In the limit $\gamma \rightarrow 0$, the resulting lineshape for the Raman spectrum, studied 
using non-polarized light, is

$$
\begin{aligned}
\mathcal{L}(\omega) & =\sum_{ \pm} \frac{3 c_{g}}{128}\left(\frac{e^{2} \Omega_{f} g_{L}^{2} L^{2}}{v c^{2} \gamma N}\right)^{2} \frac{\omega}{12 \pi \Gamma \rho_{\mathrm{L}}^{2} q_{0}^{4}} \\
& \times \frac{\left[\left(\Gamma \pm \rho_{\mathrm{L}} q_{0}^{2}\right)^{2}-s_{\mathrm{L}}^{2} q_{0}^{2}\right]^{2}}{\sqrt{\left[\Gamma^{2}-q_{0}^{2}\left(s_{\mathrm{L}}-\rho_{\mathrm{L}} q_{0}\right)^{2}\right]\left[q_{0}^{2}\left(s_{\mathrm{L}}+\rho_{\mathrm{L}} q_{0}\right)^{2}-\Gamma^{2}\right]}}, \\
\Gamma(\omega) & =\left|\omega_{\mathrm{L}}^{2}+\lambda_{\mathrm{L}} q_{0}^{2}-\omega^{2} / 4\right|, q_{0} \approx \Omega_{i} / v,
\end{aligned}
$$

where $c_{g}=\left\langle\left[\mathbf{e}_{\mathbf{q}} \times \mathbf{e}_{i}\right]_{z}^{2}\left[\mathbf{e}_{\mathbf{q}} \times \mathbf{e}_{f}^{*}\right]_{z}^{2}\right\rangle \sim 1 / 4$ is a factor determined by averaging over the photon polarization. The lineshape is composed of two non-overlapping lines corresponding to the $\mathrm{LA}(+)$ and $\mathrm{LO}(-)$ phonon branches. The contribution from each line is characterized by a square root singularity $\propto\left[\omega-\omega_{ \pm, l}\right]^{-1 / 2}$ at the lower spectral end, $\omega_{ \pm, l}=2\left[\omega_{\mathrm{L}}^{2}+\lambda_{\mathrm{L}} q_{0}^{2} \mp s_{\mathrm{L}} q_{0}-\rho_{\mathrm{L}} q_{0}^{2}\right]^{1 / 2}$, and it vanishes as $\left[\omega_{ \pm, u}-\omega\right]^{3 / 2}$ at the upper spectral end, $\omega_{ \pm, u}=2\left[\omega_{\mathrm{L}}^{2}+\lambda_{\mathrm{L}} q_{0}^{2} \mp s_{\mathrm{L}} q_{0}+\rho_{\mathrm{L}} q_{0}^{2}\right]^{1 / 2}$. In experiments, each contribution would acquire a distinctive triangular shape, smeared by finite broadening $\sim \gamma s_{\mathrm{L}} / v$. The splitting $\sim 2 s_{\mathrm{L}} \Omega_{i} / v$ and linewidth $\sim 2 \rho_{L} \Omega_{i}^{2} / v^{2}$ are smaller, and the intensities of the LA and LO modes are closer for a Raman signal taken using photons with a lower energy (FIR), whereas the LA mode should dominate in Raman spectra taken using more energetic photons (e.g. ultraviolet). Note that the TO mode near the BZ corner is strongly red-shifted by the electron-phonon interaction $[100-103,114]$, so it is possible that its spectral line will lie over that of the LO mode taken using high-energy photons.

Integrating the spectral density with respect to frequency $\omega$ yields the total intensity of the two-phonon Raman signal for longitudinal phonons near the BZ corner $I_{\mathrm{L}} \approx\left(3 c_{g} / 128\right)\left(e^{2} \Omega_{f} g_{L}^{2} L^{2} / v c^{2} \gamma N\right)^{2}$. It can be used to measure the value of the e-ph coupling constant $g_{\mathrm{L}}$ because the constant $g_{\Gamma}$ of the electron coupling to the $\Gamma$-point optical phonons has already been extracted from the density-dependent shift $[109,112,113]$ and splitting at high magnetic field [116] caused by such coupling to the electronic excitations. One can, therefore, compare $\ddagger$ the predicted

\footnotetext{
${ }^{\ddagger} I_{\mathrm{L}} / I_{\Gamma} \sim(3 / 32)\left(\omega_{\Gamma} / \omega_{\mathrm{L}}\right)^{2}\left(\epsilon^{\prime} / 3 t^{\prime}\right)^{4} \sim 1 / 140$ according to the LDA results in Table 1.
} 


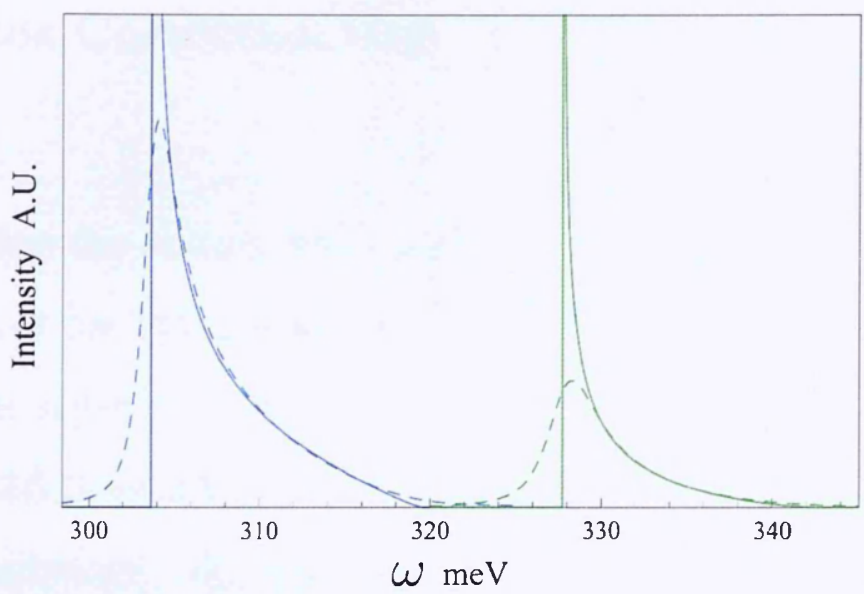

Figure 8.3: The theoretical lineshapes for the LA mode (blue lines) and LO mode (green lines). Solid lines indicate the $\gamma \rightarrow 0$ limit with the dashed lines indicating the same line shape subjected to finite broadening.

LO-LA peak intensity with that of the so-called 2D' Raman peak (corresponding to the emission of two $\Gamma$-point phonons), and determine $g_{\mathrm{L}}$ from the integral intensity ratio $I_{\mathrm{L}} / I_{\Gamma}=(3 / 32)\left(g_{\mathrm{L}} / g_{\Gamma}\right)^{4}$.

\subsection{Renormalisation of the $\mathrm{TO}_{K_{ \pm}}$Phonon for Graphene}

\section{on $\mathrm{hBN}$}

Motivated by the recent experimental measurements by A. Eckmann and coworkers [117], we now turn our attention to the Raman spectra of the graphene/hBN heterostructure. Rather than calculate the correction to the small LO-LA peak, due to the superlattice perturbation, we instead concentrate on the strongest Raman peak: the $2 \mathrm{D}$ peak. This peak is caused by the emission of a pair of $\mathrm{TO}_{K_{ \pm}}$ phonons, whose highly isotropic spectrum results in the sharp, symmetric Raman peak clearly visible in Fig. 8.1. In the absence of the superlattice, the isotropic nature of the Dirac electrons and the strong electron-phonon coupling leads to a strong isotropic downwards renormalisation of the TO phonon energy [100]. In this section I describe how the superlattice breaks the isotropy of the renormalisation, and the manifestation of this in the Raman signal. $\S$

\footnotetext{
${ }^{\S}$ A full account of the Raman signal of this system should also account for a periodic inhomogeneity of the graphene layer and corresponding shift in the $2 \mathrm{D}$ peak position (e.g. caused
} 


\subsubsection{Vertex Correction Due to the Superlattice Perturba- tion}

Before calculating the phonon self-energy diagrams, responsible for the energy renormalisation of the $\mathrm{TO}_{K_{ \pm}}$mode, we must first calculate the correction to EPI (8.4) due to the superlattice perturbation. To do this we use the point charge model (section 2.6.1) describing the perturbation to the graphene electrons due to the hexagonal substrate. The difference here is that a small displacement of the graphene lattice due to $\mathrm{TO}_{K_{ \pm}}$phonons is taken into account. Retaining terms to first order in the displacements (which we then quantise) results in the correction to the EPI,

$$
\begin{aligned}
& H_{E P M}=-2 K\left[u_{T O, K_{+}} \sigma_{z} \tau^{-}+u_{T O, K_{-}} \sigma_{z} \tau^{+}\right]\left[u_{0} v b f_{1}+u_{1} v\left[\boldsymbol{l}_{z} \times \nabla f_{2}\right] \cdot \boldsymbol{\sigma} \tau_{3}+u_{2} v \nabla f_{2} \cdot \boldsymbol{\sigma} \tau_{3}\right] \\
& u_{T O, K_{ \pm}}(\boldsymbol{r})=\sum_{\boldsymbol{q}} \frac{1}{\sqrt{2 N m \omega_{T O}}}\left(b_{-\left(K_{ \pm}+\boldsymbol{q}\right)}^{\dagger}+b_{K_{ \pm}+q}\right) e^{i \boldsymbol{q} \cdot \boldsymbol{r}}
\end{aligned}
$$

Here $K=\left|\boldsymbol{K}_{ \pm}\right|$and parameters $u_{i}$ retain their meanings from the point charge model in section 2.6.1

\subsection{2 $\mathrm{TO}_{K_{ \pm}}$Self-Energy Calculation}

We calculate the renormalisation of the $\mathrm{TO}_{K_{ \pm}}$phonon energy due to the self-energy diagrams, shown graphically in Fig. 8.4. We treat the superlattice perturbation as small and therefore only include diagrams up to second order in this parameter. There is no correction to first order since the moiré perturbation transfers momentum $\boldsymbol{b}_{m}$ to the electron, which must latter be absorbed.

In Figure 8.4, diagram $\Pi_{0, A}$ represents the usual isotropic correction to the

by strain modulated with the periodicity of the moiré pattern). The size of laser spot used in experiments averages over many moiré periods which leads to a broadening of the Raman signal [117].

ISince Hamiltonian (8.8) consists of the product of a superlattice perturbation in the form of Eq. (2.2) and the EPI for the $\mathrm{TO}_{K_{ \pm}}$phonon (8.4), it is trivial to check that it obeys the appropriate geometrical symmetries. A term like the $u_{3}$ perturbation in Eq. (2.2) can not be added into Eq. (8.8) since it would not be Hermitian. Also the $u_{2}$-like term in (8.8) can not be gauged away. 


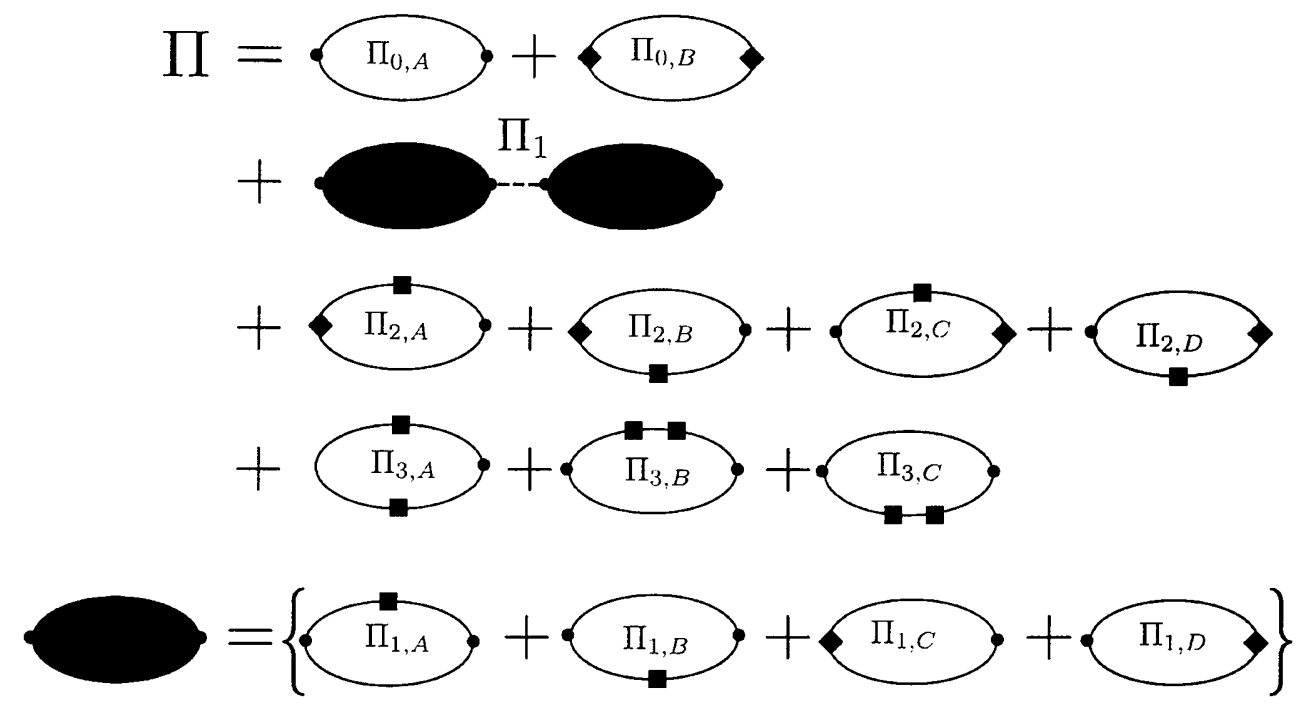

Figure 8.4: Contributions to the self energy of the $\mathrm{TO}_{K_{ \pm}}$phonon, taking into account contributions up to second order in the superlattice perturbation. Solid (dashed) lines represent the unperturbed electron (phonon) Green's function, circles represent the EPI for the $\mathrm{TO}_{K_{ \pm}}$phonon (Eq. 8.4), diamonds the vertex correction to the EPI (Eq. 8.8), and black squares the moiré perturbation (Eq. 2.2).

$\mathrm{TO}_{K_{ \pm}}$energy. This part, calculated in Ref. [100], is always present regardless of the substrate and therefore we assume that it is already accounted for in the energy of the $\mathrm{TO}_{K_{ \pm}}$phonon. In contrast, diagram $\Pi_{0, B}$ does depend on the substrate. However this diagram is also neglected since it only leads to a small featureless correction to the contribution from $\Pi_{0, A}$. Also a cancellation between the diagrams $\prod_{2, i=A, B, C, D}$ means that their contribution vanishes. Finally, the intermediate phonon state in $\Pi_{1}$ is almost on resonance, and therefore the contribution due to $\Pi_{1}$ is far greater than that due to $\Pi_{3, i=A, B, C}$. This allows $\Pi_{3, i}$ to be neglected"

Therefore, keeping terms up to $q^{2}$, the dominant contribution to the phonon

\footnotetext{
$\|$ Actually this diverges at small $b=\left|b_{m}\right|$ as $\Pi_{3, i}\left(\omega_{\mathrm{TO}}(\boldsymbol{q}), \boldsymbol{q}\right) \sim \frac{3 \sqrt{3} a_{c c}^{2} g_{T O}^{2}}{\pi v^{2}} \frac{\left(v b u_{i}\right)^{2}}{v q} \log \left(\frac{q}{b}\right)$. However, even for perfect alignment between graphene and hBN, $b$ takes a finite value of approximately $0.052 \AA$. Therefore setting $q=0.3 \AA$ (typical wavevector of phonon emitted in a Raman experiment) and $v b u_{i} \sim 30 \mathrm{meV}$ we estimate $\Pi_{3, i}(\boldsymbol{q}) \lesssim 0.03 \mathrm{meV}$. This is negligible on the scale of Fig. 8.5 and has been confirmed by numerical calculations.
} 
self-energy correction is,

$$
\begin{gathered}
\Pi_{1}\left(\omega_{\mathrm{TO}}(\boldsymbol{q}), \boldsymbol{q}\right) \approx \sum_{m=0,2,4} \frac{\left|\Pi_{1, A}(\boldsymbol{q})+\Pi_{1, B}(\boldsymbol{q})+\Pi_{1, C}(\boldsymbol{q})+\Pi_{1, D}(\boldsymbol{q})\right|^{2}}{v_{T O} q \cot ^{2}\left(\theta^{\prime}\right)-v_{T O} \frac{b^{2}}{4 q} \sin ^{2}\left(\theta^{\prime}\right)} \\
\Pi_{1, A}(\boldsymbol{q})+\Pi_{1, B}(\boldsymbol{q}) \approx \frac{12 \sqrt{3} a_{c c}^{2} g_{T O}^{2}}{v^{2}(2 \pi)^{2}} I_{A B}\left[v b u_{1} \sin \left(\theta^{\prime}\right)+v b u_{2} \cos \left(\theta^{\prime}\right)\right] \\
\Pi_{1, C}(\boldsymbol{q})+\Pi_{1, D}(\boldsymbol{q}) \approx \frac{12 \sqrt{3} a_{c c}^{2} g_{T O}|K|}{\sqrt{2 m \omega_{T O}}} \frac{v b u_{0}}{v^{2}(2 \pi)^{2}}\left[2 \pi \Lambda-\frac{\pi^{2}}{2} v q+I_{C D} \frac{(v q)^{2}}{\Lambda}\right]
\end{gathered}
$$

Here $\theta^{\prime} \equiv \theta_{\boldsymbol{q}}-\frac{2 \pi m}{6}-\theta_{\boldsymbol{b}_{0}}, \theta_{\boldsymbol{q}\left(\boldsymbol{b}_{0}\right)}$ is the angle $\boldsymbol{q}\left(\boldsymbol{b}_{0}\right)$ makes with the $\mathrm{x}$-axis, and $\Lambda$ is a high energy cut-off, of order the graphene $\pi$-band width, which was introduced to ensure convergence of the integral over intermediate electron states. Also the energy scales $v b$ and $\omega_{T O}$ were neglected in comparison to the typical energies of the intermediate electrons, and the dimensionless integrals $I_{A B} \approx 2.44$ and $I_{C D} \approx$ 1.17 where calculated numerically. The phonon Green's function $D^{0}(\omega, \boldsymbol{q})$ was approximated as $D^{0}\left(\omega_{T O}(\boldsymbol{q}), \boldsymbol{q}+\boldsymbol{b}_{m}\right) \approx\left[\omega_{T O}(\boldsymbol{q})-\omega_{T O}\left(\boldsymbol{q}+\boldsymbol{b}_{m}\right)+i 0\right]^{-1}$. Moreover, since Raman line-shape only depends on the phonon spectra around $q \approx q_{0} \approx \Omega_{i} / v$, the almost isotropic phonon dispersion has been approximated by $\omega_{T O}(\boldsymbol{q}) \approx v_{T O}|q|$ with $v_{T O} \approx 0.035 \mathrm{eV} \AA$ (using Ref. [114] and $\Omega_{i} \approx 2 \mathrm{eV}$ ).

The TO mode energy $\omega_{T O}\left(q_{0}, \theta_{q}\right)$ for $\Omega_{i} \approx 2 \mathrm{eV}$, subject to the correction in Eq. (8.9), is displayed in the left panel Fig. 8.5 for various misalignment angles, $\theta$, of the graphene and its substrate. In contrast to the unperturbed spectra, a strong angular dependence with a $2 \pi / 3$ period is evident. This periodicity, rather than a $2 \pi / 6$ periodicity, reflects the fact that both $\Pi_{1, A}+\Pi_{1, B}$ and $\Pi_{1, C}+\Pi_{1, D}$ make a significant contribution to $\Pi_{1}$. The right panel displays the corresponding Raman spectra, calculated using the method outlined in section 8.4. A pair of small satellite peaks is visible at small misalignment angles, the splitting of which reflects the $2 \pi / 3$ periodicity in the spectrum. 

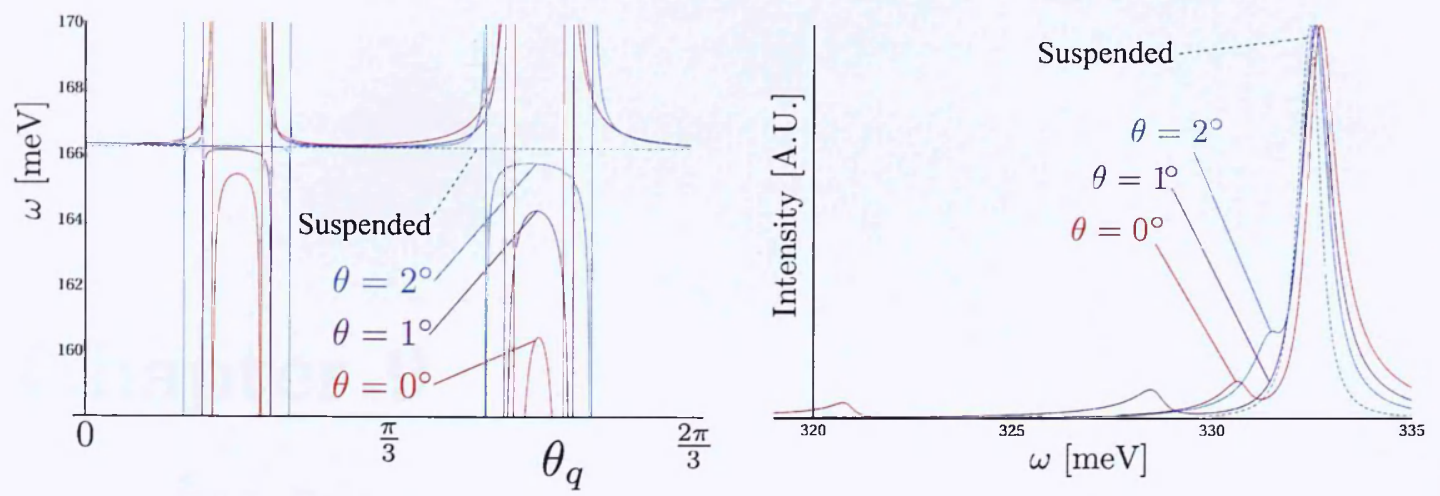

Figure 8.5: The $\mathrm{TO}_{K_{+}}$phonon energy $\omega_{T O}\left(q_{0}, \theta_{q}\right)$ (left panel) is displayed for various misalignment angles, $v q_{0}=2 \mathrm{eV}$, and $\Lambda=5 \mathrm{eV}$. The spectrum in the absence of the superlattice perturbation is assumed to be isotropic. Superlattice parameters from the microscopic model (section 2.6.1), scaled so that $\left|u_{0}\right|+\left|u_{1}\right|+\left|u_{3}\right|=0.15$, have been used. The corresponding 2D Raman peaks are shown in the right panel.

\subsection{Conclusion}

We used a minimal tight-binding model for the EPI with the $\mathrm{LA}_{\mathrm{K}_{ \pm}}$and $\mathrm{LO}_{\mathrm{K}_{ \pm}}$ phonons in the BZ corner in graphene to study the resonant two-phonon Raman processes. Owing to the strong anisotropy of the nearly degenerate $\mathrm{LA}_{\mathrm{K}_{ \pm}}$and $\mathrm{LO}_{\mathrm{K}_{ \pm}}$branches, the predicted contribution to the Raman spectrum of graphene is non-Lorentzian (almost triangular) with a much larger linewidth than other twophonon peaks that is strongly dependent on the energy of the incoming photon. We also show that the presence of a superlattice perturbation modifies the EPI, resulting in a non-isotropic renormalisation of the energy of the $\mathrm{TO}_{\mathrm{K}_{ \pm}}$mode. 


\section{Chapter 9}

\section{Conclusion}

In the preceding chapters, we have developed the theory of graphene heterostructures, and described how the novel features of these systems are manifested in experiment. Chapter 2 dealt with the reconstruction of graphene's spectra due to the superlattice potential arising from an hBN underlay. The generic appearance of an isolated secondary Dirac point on the edge of the first miniband was predicted. This feature was observed experimentally, by Yankowitz and co-workers [22], as a dip in the density of states, on the valence band side of graphene's spectrum. The recent transport measurements [23], described in chapter 5, measured a change in sign of the Hall resistivity in graphene's valence band. This confirmed the presence of the electron-like carriers associated with the isolated secondary Dirac point. Moreover, the experimental manifestations of the fractal magnetic minibands spectrum were also observed. Here, the tendency of the magnetic minibands to form strong Dirac-like features (chapter 4), at simple flux fractions was observed as oscillations of the Hall resistivity, periodic in the inverse of the magnetic field strength. Similar results were later reported, for unencapsulated graphene-hBN heterostructures, by Hunt and Co-workers [25]. The principle difference with Ref. [23] being the appearance of a small gap in the main Dirac point.

Chapter 6 dealt with the bilayer-graphene/hBN heterostructure, predicting the appearance of a gap, on the edge of the first miniband, for a broad range of 
superlattice parameters. However, the pair of microscopic models, based on either scattering by the quadrupole electric moments of nitrogen, or the hopping between carbon and $\mathrm{hBN}$ atoms, predict the exceptional choice of superlattice parameters for which the first miniband edge is almost gapless. This is in agreement with the experimental results of Dean and co-workers [24], for which no significant gap was observed, and lends credit to the use of the microscopic models.

An alternative method of narrowing down the superlattice parameters is proposed in chapter 3, based on the anticipated modulation of graphene's optical absorption spectra. The effect of each symmetry allowed superlattice perturbation term is analysed in detail, which should prove a useful guide to experiment.

Further experiments are proposed in chapter 7 . Here we show that large band gaps may be generated, in graphene's bandstructure, when placed on an hexagonal underlay almost commensurate with the $\sqrt{3} \times \sqrt{3}$ Kekulé lattice of graphene. We propose that the (111)B surface of InAs is suitable for this role.

Separately, in chapter 8, we analyze the electron-phonon coupling and Raman spectra of both graphene and graphene/hBN heterostructures. Raman processes accompanied by the emission of a pair of LO or LA phonons from the corner of the Brillouin zone are included, and we find that the lineshape of the corresponding Raman signal consists of two peaks with a strongly non-Lorentzian (almost triangular) form. 


\section{Appendix A}

\section{Minibands in the Vicinity of $\kappa$}

\section{and $\mu$-points of Graphene-hBN}

\section{Heterostructures}

Additional terms can be included in effective Hamiltonians Eq. (2.5) and Eq. (2.6) of the main text to increase their accuracy and provide greater detail on the movement of the sDPs.

\section{A.1 $\mu$-point Hamiltonian}

Similar to Eq. (2.6), we project Hamiltonian (2.2) on to the basis, $(|\boldsymbol{\mu}+\boldsymbol{q}\rangle, \mid \boldsymbol{\mu}+$ $\left.\boldsymbol{b}_{3}+\boldsymbol{q}\right\rangle$ ). Retaining terms to second order in $u_{i}$ and first order in $\tilde{u}_{i}$,

$$
\begin{aligned}
& \hat{H}_{\boldsymbol{\mu}+\boldsymbol{q}}=v b\left(\begin{array}{cc}
E_{\mu}+s \frac{q_{y}}{b} & H_{12} \\
H_{12}^{*} & E_{\mu}-s \frac{q_{y}}{b}
\end{array}\right) \\
& E_{\mu}=s\left(1 / 2+q_{x}^{2} / b^{2}-\frac{1}{6} s\left(24 u_{0}^{2}+29 u_{1}^{2}+5 u_{3}^{2}-70 s u_{1} u_{3}\right)\right. \\
& H_{12}=\left(s u_{1}-u_{3}\right)-i\left(s \tilde{u}_{1}-\tilde{u}_{3}\right)+4 u_{0}\left(2 u_{1}-s u_{3}\right)+2 \frac{q_{x}}{b}\left(u_{0}+i \tilde{u}_{0}\right)+2 \frac{q_{x}^{2}}{b^{2}}\left(u_{3}-i \tilde{u}_{3}\right) .
\end{aligned}
$$


The spectrum resulting from Eq. (A.1) contains two anisotropic sDPs, rather than the one predicted by Eq. (2.5), which occur at wavevectors,

$$
q_{y}^{\mathrm{sDP}}=0, \quad q_{x}^{\mathrm{sDP}}=\frac{-u_{0} \pm \sqrt{u_{0}^{2}+2 u_{3}\left(-s u_{1}+u_{3}-4 u_{0}\left(2 u_{1}-s u_{3}\right)\right)}}{2 u_{3}} b
$$

When $\left|u_{0}\right| \gg\left|u_{1}\right|,\left|u_{3}\right|$, the two wavevectors are $\boldsymbol{q}^{\mathrm{sDP}} \approx\left(\left[u_{3}-s u_{1}\right] /\left[2 u_{0}\right], 0\right) b$ and $\approx\left(-u_{0} / u_{3}, 0\right) b$. Only the former occurs within the range of momenta for which $\hat{H}_{\boldsymbol{\mu}+\boldsymbol{q}}$ is valid, and coincides with the single sDP depicted in the top line of Fig. 2.2. Away from the $\left|u_{0}\right| \gg\left|u_{1}\right|,\left|u_{3}\right|$ limit, $\hat{H}_{\mu+q}$ can capture the merging of two sDPs of opposite topological winding number [70,131], which occurs when $u_{0}^{2}+2 u_{3}\left(-s u_{1}+u_{3}-4 u_{0}\left(2 u_{1}-s u_{3}\right)\right)=0$.

It is an interesting technical point that, to capture the existence an ungapped sDP in Eq. (A.1), requires the existence of a particular $q_{x}$ for which both the real and imaginary parts of $H_{12}$ simultaneous vanish. For an approximate Hamiltonian, such as $\hat{H}_{\mu+q}$, this is not guaranteed. Indeed, due to the expansion in $q$, the presence or absence of ungapped sDPs depends on the ( $q$-dependent) gauge choice used for the Dirac plane wave states in the basis of $\hat{H}_{\mu+q}$. In Eq. (A.1) (and also Eq. (A.3) ) the careful use of Dirac plane waves in the gauge $\left(e^{-i \theta_{\boldsymbol{q}} / 2}, s e^{i \theta_{\boldsymbol{q}} / 2}\right)^{T}$ was required.

\section{A.2 Hamiltonian at the $\kappa$-point}

In a similar manner to Eq. (2.6) we take a basis of graphene plane waves $(\mid \zeta(\kappa+$ $\left.\boldsymbol{q})\rangle,\left|\zeta\left(\boldsymbol{\kappa}+\boldsymbol{b}_{1}+\boldsymbol{q}\right)\right\rangle,\left|\zeta\left(\boldsymbol{\kappa}+\boldsymbol{b}_{2}+\boldsymbol{q}\right)\right\rangle\right)$, however now terms to second order in $u_{i}$ and 
first order in $\tilde{u}_{i}$ are retained,

$$
\begin{gathered}
H_{\zeta(\boldsymbol{\kappa}+\boldsymbol{q})}=v b\left(\begin{array}{ccc}
E_{\kappa}+\frac{s q_{x}}{b} & \left(w_{\zeta}+v_{u} Q_{+}\right) e^{i \frac{2 \pi}{6}} & \left(w_{\zeta}^{*}+v_{u} Q_{-}\right) e^{-i \frac{2 \pi}{6}} \\
\left(w_{\zeta}^{*}+v_{u} Q_{+}\right) e^{-i \frac{2 \pi}{6}} & E_{\kappa}-s Q_{-} & \left(w_{\zeta}-v_{u} \frac{q_{x}}{b}\right) e^{i \frac{2 \pi}{6}} \\
\left(w_{\zeta}+v_{u} Q_{-}\right) e^{i \frac{2 \pi}{6}} & \left(w_{\zeta}^{*}-v_{u} \frac{q_{x}}{b}\right) e^{-i \frac{2 \pi}{6}} & E_{\kappa}-s Q_{+}
\end{array}\right), \\
E_{\kappa}=\frac{s}{\sqrt{3}}\left(1-\frac{21}{4} u_{0}^{2}-3 u_{1}^{2}+\frac{9}{4} u_{3}^{2}-6 s \zeta u_{0} u_{1}+\frac{18 s}{\sqrt{3}} u_{1} u_{3}+\frac{9}{2 \sqrt{3}} \zeta u_{0} u_{3}\right) \\
w_{\zeta}=\frac{1}{2}\left[\left(u_{0}-2 s \zeta u_{1}+\sqrt{3} \zeta u_{3}\right)+i \zeta\left(\tilde{u}_{0}+2 s \zeta \tilde{u}_{1}-\sqrt{3} \zeta \tilde{u}_{3}\right)\right. \\
\left.-\frac{s}{2}\left(\frac{11 u_{0}^{2}}{2 \sqrt{3}}+\frac{10 u_{1}^{2}}{\sqrt{3}}+\frac{9 u_{3}^{2}}{2 \sqrt{3}}+\frac{20 \zeta s u_{0} u_{1}}{\sqrt{3}}-4 s u_{1} u_{3}-\zeta u_{0} u_{3}\right)\right] \\
v_{u}=\frac{3}{4}\left(\sqrt{3} u_{0}-\zeta u_{3}\right), \quad Q_{ \pm}=\frac{\left(q_{x} \pm \sqrt{3} q_{y}\right)}{2 b} .
\end{gathered}
$$

In the absence of inversion-asymmetric terms, the $\boldsymbol{q}=0$ spectra results in a singlet and a doublet band at energies $E_{\kappa}-2 w_{\zeta}$ and $E_{\kappa}+w_{\zeta}$ respectively, so that sDP is always seen in the doublet band at the exact $\zeta \kappa$-point, with Dirac velocity $\left[1+2 v_{u}\right] \frac{v}{2}$.

Away from the exact $\kappa$-point, three additional sDPs are found with wavevector and energy,

$$
\begin{aligned}
& q_{y}^{\mathrm{sDP}}=0, \quad q_{x}^{\mathrm{sDP}}=\frac{2 w_{\zeta}\left(s+2 v_{u}\right)}{v_{u}\left(2 s+v_{u}\right)} \approx w_{\zeta} / v_{u} \\
& \epsilon=E_{\kappa}+w_{\zeta}-\left(\frac{s}{2}+v_{u}\right) q_{x}^{\mathrm{sDP}} \approx E_{\kappa}+w_{\zeta}-s \frac{w_{\zeta}}{2 v_{u}},
\end{aligned}
$$

as well as the wavevectors obtained by a $\pm 2 \pi / 3$ rotation. For $v_{u}>0$ these sDP occur between the singlet and the doublet band, where as for $v_{u}<0$ they occurs between the two branches of the doublet band and correspond to the sDPs obtained from Eq. (A.1). 


\section{Appendix B}

\section{Minibands of Graphene with}

\section{Almost Commensurate $\sqrt{3} \times \sqrt{3}$ \\ Hexagonal Crystals}

The minibands described by Hamiltonian (7.4) of chapter 7 will now be discussed in greater detail. The minibands can feature secondary Dirac Points (sDPs), which we also discuss. However, in contrast to the graphene-hBN heterostructures (chapter 2 ), the sDPs discussed here never become isolated on the energy axis for any reasonable choice of perturbation parameters.

For $\boldsymbol{k}=\boldsymbol{b}_{0} / 2$, zone folding brings together two states, one from each valley, each with energy $s \frac{v b}{2 \sqrt{3}}$. Their splitting is described by,

$$
\epsilon_{s, \pm}=\frac{s v b}{\sqrt{3}}\left(\frac{1}{2} \pm 2\left(U_{E^{\prime}}-s U_{G}\right)+\frac{4}{3}\left(2 U_{E^{\prime}}^{2}+8 s U_{E^{\prime}} U_{G}-U_{G}^{2}\right)\right) .
$$

When $U_{E^{\prime}}=s U_{G}$ the degeneracy of these states remains intact, as shown in the conduction band in Fig. B.1 (a). For $\left|U_{E^{\prime}}\right|<\left|U_{G}\right|$ the degeneracy is lifted such that the two lowest energy minibands do not touch at any point. For $\left|U_{E^{\prime}}\right| \gtrsim\left|U_{G}\right|$ the degeneracy is also lifted but the two band touch at six highly anisotropic sDPs (one of which is circled in Fig. B.1 (b)). The sDPs travel in a straight line towards $k=0$ as $\left|U_{E^{\prime}}\right|$ is increased relative to $\left|U_{G}\right|$. When $0 \leq U_{E^{\prime}}^{2}-U_{G}^{2} \ll 1$, their positions 
are given by $\boldsymbol{k} \approx \boldsymbol{b}_{0} / 2-\frac{\sqrt{U_{E^{\prime}}^{2}-U_{G}^{2}}}{2 \sqrt{3}\left|U_{G}\right|}$ by and $2 \pi / 6$ rotations thereof. It can also be noted that the Van Hove singularities, surrounding the main Dirac points in the lower two panels of Fig. 7.2 , arise due to the saddle point at $\boldsymbol{k}=\boldsymbol{b}_{0} / 2$ (related by $2 \pi / 3$ rotations) in the dispersion of the lowest energy branch described by Eq. B.1.

At $k=0$, the zone folding brings together 3 states from each valley, each with an energy $\frac{s v b}{\sqrt{3}}$. These are split into two nondegenerate states, $\epsilon_{s, \pm}^{(1)}$, and two two-fold degenerate states, $\epsilon_{s, \pm}^{(2)}$, with energies,

$$
\begin{aligned}
\epsilon_{s, \pm}^{(1)} & =\frac{s v b}{\sqrt{3}}\left(1 \pm 2\left(U_{E^{\prime}}-2 s U_{G}\right)+\frac{4}{3}\left(U_{E^{\prime}}+s U_{G}\right)^{2}+4 U_{G^{\prime}}^{2}\right) \\
\epsilon_{s, \pm}^{(2)} & =\frac{s v b}{\sqrt{3}}\left(1 \pm\left(U_{E^{\prime}}-2 s U_{G}\right)+\frac{4}{3}\left(U_{E^{\prime}}+s U_{G}\right)^{2}+\frac{9}{2} U_{E}^{2}-2 U_{G^{\prime}}^{2}\right) .
\end{aligned}
$$

The 6 anisotropic sDPs will reach $k=0$ at $U_{E^{\prime}}=2 s U_{G}$ which is when the two nondegenerate states, $\epsilon_{s, \pm}^{(1)}$, cross. This allows the first and second minibands to separate from each other, allowing a global bandgap to develop for a sufficiently strong perturbation.

a)

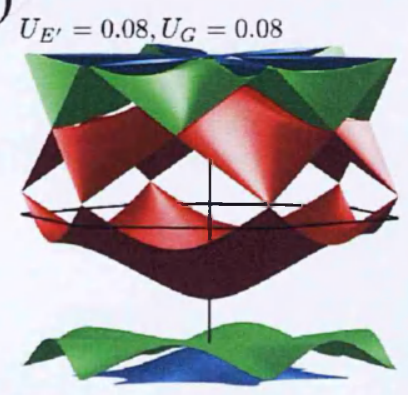

b)

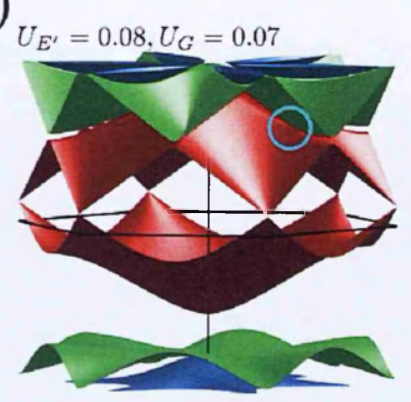

c)

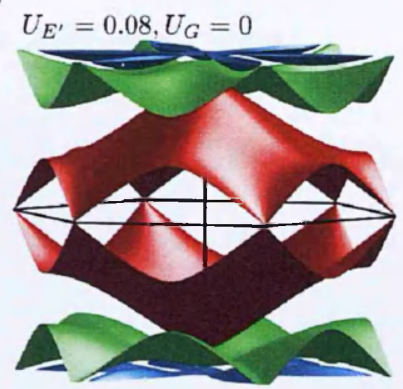

Figure B.1: Further examples of numerically calculated moiré minibands shown in the larger mBZ. Showing perturbation parameters for which; (a) $\boldsymbol{k}=\boldsymbol{b}_{0} / 2$ is degenerate; (b) 6 highly anisotropic sDPs are found on the mBZ edge; (c) the first and second minibands are not connected, also note the electrons-hole symmetry (discuss in section 7.6) is present in this case. 


\section{Bibliography}

[1] P. R. Wallace, Phys. Rev. 71, 622 (1947).

[2] J. W. McClure, Phys. Rev. 108, 612 (1957).

[3] J. C. Slonczewski and P. R. Weiss, Phys. Rev. 109, 272 (1958).

[4] H. W. Kroto, J. R. Heath, S. C. O'Brien, R. F. Curl, and R. E. Smalley, Nature 318, 56 (1985).

[5] S. Ijima, Nature 354, 56 (1991).

[6] K. S. Novoselov, A. K. Geim, S. V. Morozov, D. Jiang, Y. Zhang, S. V. Dubonos, I. V. Grigorieva, A. A. Firsov, Science 306, 666 (2004).

[7] K. S. Novoselov, D. Jiang, F. Schedin, T. J. Booth, V. V. Khotkevich, S. V. Morozov, and A. K. Geim, Proc. Natl. Acad. Sci. USA 102, 10451 (2005).

[8] A. K. Geim, I. V. Grigorieva, Nature 499, 419 (2013).

[9] C. R. Dean, A. F. Young, I. Meric, C. Lee, L. Wang, S. Sorgenfrei, K. Watanabe, T. Taniguchi, P. Kim, K. L. Shepard, and J. Hone, Nat. Nano. 5, 722 (2010).

[10] J. Xue, J. Sanchez-Yamagishi, D. Bulmash, P. Jacquod, A. Deshpande, K. Watanabe, T. Taniguchi, P. Jarillo-Herrero, and B. J. LeRoy, Nat. Mater. 10, 282 (2011). 
[11] R. Decker, Y. Wang, V. W. Brar, W. Regan, H.-Z. Tsai, Q. Wu, W. Gannett, A. Zettl, and M. F. Crommie, Nano Lett. 11, 2291 (2011).

[12] A. S. Mayorov, R. V. Gorbachev, S. V. Morozov, L. Britnell, R. Jalil, L. A. Ponomarenko, P. Blake, K. S. Novoselov, K. Watanabe, T. Taniguchi, and A. K. Geim, Nano Lett. 11, 2396 (2011).

[13] E. Kim, T. Yu, E. S. Song, B. Yu, Appl. Phys. Lett. 98, 262103 (2011).

[14] H. Wang, T. Taychatanapat, A. Hsu, K. Watanabe, T. Taniguchi, P. JarilloHerrero, and T. Palacios, IEEE Electron Device Lett. 32, 1209 (2011).

[15] M. S. Bresnehan, M. J. Hollander, M. Wetherington, M. LaBella, K. A. Thurnbull, R. Cavalero, D. W. Snyder, and J. A. Robinson, ACS Nano 6, 5234 (2012).

[16] J. C. Meyer, A. K. Geim, M. I. Katsnelson, K. S. Novoselov, T. J. Booth and S. Roth, Nature 446, 60 (2007).

[17] K. I. Bolotin, K. J. Sikes, Z. Jiang, M. Klima, G. Fudenberg, J. Hone, P. Kim, H. L. Stormer, Solid State Commun. 146, 351 (2008).

[18] C. Ortix, L. Yang, and J. van den Brink, Phys. Rev. B 86, 081405 (2012).

[19] M. Kindermann, B. Uchoa and D. L. Miller, Phys. Rev. B 86, 115415 (2012).

[20] C.-H. Park, L. Yang, Y.-W. Son, M. L. Cohen and S. G. Louie, Nature Phys. 4, $213(2008)$.

[21] J. R. Wallbank, A. A. Patel, M. Mucha-Kruczynski, A. K. Geim, V. I. Fal'ko, Phys. Rev. B 87, 245408 (2013).

[22] M. Yankowitz, J. Xue, D. Cormode, J. D. Sanchez-Yamagishi, K. Watanabe, T. Taniguchi, P. Jarillo-Herrero, P. Jacquod, and B. J. LeRoy, Nature Phys. 8, $382(2012)$. 
[23] L. A. Ponomarenko, R. V.Gorbachev, G. L.Yu, D. C. Elias, R. Jalil, A. A. Patel, A. Mishchenko, A. S. Mayorov, C. R. Woods, J. R. Wallbank, M. MuchaKruczynski, B. A. Piot, M. Potemski, I. V. Grigorieva, K. S. Novoselov, F. Guinea, V. I. Fal'ko, A. K. Geim, Nature 497, 594 (2013).

[24] C. R. Dean, L. Wang, P. Maher, C. Forsythe, F. Ghahari, Y. Gao, J. Katoch, M. Ishigami, P. Moon, M. Koshino, T. Taniguchi, K. Watanabe, K. L. Shepard, J. Hone, P. Kim, Nature 497, 598 (2013).

[25] B. Hunt, J. D. Sanchez-Yamagishi, A. F. Young, M. Yankowitz, B. J. LeRoy, K. Watanabe, T. Taniguchi, P. Moon, M. Koshino, P. Jarillo-Herrero, R. C. Ashoori, Science 340, 1427 (2013).

[26] W. Yang, G. Chen, Z. Shi, C-C Liu, L. Zhang, G. Xie, M. Cheng, D. Wang, R. Yang, D. Shi, K. Watanabe, T. Taniguchi, Y. Yao, Y. Zhang and G. Zhang, Nature Mat. 12, 792 (2013).

[27] R. Saito, G. Dresselhaus,M. S. Dresselhaus, Physical Properties of Carbon Nanotubes, (Imperial College Press, 1998).

[28] A. H. Castro Neto, F. Guinea, N. M. R. Peres, K. S. Novoselov and A. K. Geim, Rev. Mod. Phys. 81, 109 (2009).

[29] J. C. Slater and G. F. Koster, Phys. Rev.94, 1498 (1954).

[30] E. McCann in Graphene Nanoelectronics: Metrology Synthesis, Properties and Applications, edited by Hassan Raza, (Springer-Verlag, 2012).

[31] A. A. Abrikosov, Fundamentals of the Theory of Metals, translated by A. Beknazarov (Elsevier Science Publishers, 1998).

[32] J.M. Luttinger and W. Kohn, Phys. Rev. 97, 869 (1955).

[33] D.P. DiVincenzo and E.J. Mele, Phys. Rev. B 29, 1685 (1984).

[34] T. Ando, J. Phys. Soc. Jpn. 74, 777 (2005). 
[35] E. McCann and V. I. Falko, Phys. Rev. Lett. 96, 086805 (2006)

[36] G.L.Bir and G.E.Pikus, Symmetry and strain-induced effects in semiconductors, (John Wiley and Sons, 1974).

[37] M. Tinkham Group theory in quantum mechanics, (McGraw-Hill, Inc. 1964).

[38] I. L. Aleiner and K. B. Efetov, Phys. Rev. Lett. 97, 236801 (2006).

[39] D. M. Basko, Phys. Rev. B 78, 125418 (2008).

[40] J.W. McClure, Phys. Rev. 104, 666 (1956).

[41] Y. B. Zhang, Y W. Tan, H. L. Stormer, P. Kim, Nature 438, 201 (2005).

[42] K. S. Novoselov, E. McCann, S. V. Morozov, I. V. Falko, M. I. Katsnelson, U. Zeitler, D. Jiang, F. Schedin, A. K. Geim, Nature Phys. 2, 177 (2006).

[43] J. R. Schrieffer, P. A. Wolff, Phys. Rev. 149, 491 (1966).

[44] J. Sławińska, I. Zasada, Z. Klusek, Phys. Rev. B 81, 155433 (2010).

[45] Handbook of Moiré Measurents, edited by C. A. Walker, (IOP Publishing Ltd, 2004).

[46] F. Blais, Journal of Electronic Imaging 13, 231 (2004).

[47] K. Hermann, J. Phys.: Condens. Matter. 24, 314210 (2012).

[48] A. T. NDiaye, S. Bleikamp, P. J. Feibelman and T. Michely, Phys. Rev. Lett. 97, 215501 (2006); S. Marchini, S. Günther and J. Wintterlin, Phys. Rev. B 76, 075429 (2007); P. Sutter, J. T. Sadowski, E. Sutter, Phys. Rev. B 80, 245411 (2009); M. Sicot, P. Leicht, A. Zusan, S. Bouvron, O. Zander, M. Weser, Y. S. Dedkov, K. Horn, and M. Fonin, ACS Nano 6, 151 (2012). J. Wintterlin, M.-L. Bocquet, Surface Science 603, 1841 (2009). M. Batzill, Surface Science Reports 67, 83 (2012). 
[49] J. Coraux, A. T. N'Diaye, C. Busse, and T. Michely, Nano. Lett. 8, 565 (2008).

[50] F. R. N. Nabarro, Theory of Crystal Dislocations, (Oxford university press 1967).

[51] J. M. B. Lopes dos Santos, N. M. R. Peres, and A. H. Castro Neto, Phys. Rev. Lett. 99, 256802 (2007).

[52] R. Bistritzer and A. H. MacDonald, Phys. Rev. B 81245412 (2010).

[53] R. Bistritzer and A. H. MacDonald, Phys. Rev. B 84035440 (2011).

[54] J. M. B. Lopes dos Santos, N. M. R. Peres, and A. H. Castro Neto, Phys. Rev. B 86, 155449 (2012).

[55] S. V. Iordanskii and A. Koshelev, JETP Lett. 41, 574 (1985).

[56] M. S. Foster and A. W. W. Ludwig, Phys. Rev. B 73, 155104 (2006).

[57] A. F. Morpurgo and F. Guinea, Phys. Rev. Lett. 97, 196804 (2006).

[58] C.-H. Park, L. Yang, Y.-W. Son, M. L. Cohen, and S. G. Louie, Phys. Rev. Lett. 101, 126804 (2008).

[59] F. Guinea and T. Low, Phil. Trans. R. Soc. A 368, 5391 (2010).

[60] D. R. Hofstadter, Phys. Rev. B 14, 2239 (1976).

[61] G. Giovannetti, P. A. Khomyakov, G. Brocks, P. J. Kelly, and J. van den Brink, Phys. Rev. B 76, 073103 (2007).

[62] M. Kindermann and P. N. First, Phys. Rev. B 83, 045425 (2011).

[63] P. Moon and M. Koshino, Phys. Rev. B 87, 205404 (2013).

[64] P. Moon and M. Koshino, arXiv:1308.0713 (2013).

[65] J. Zak, Phys. Rev. 134, A1602 (1964); 134, A1607 (1964). 
[66] E. Brown, Phys. Rev. 133, A1038 (1964).

[67] L. D. Landau and E. M. Lifshitz, Statistical Physics (Pergamon Press Ltd., 1980), Vol. 9 part 2, Sec. 60.

[68] D. K. Ferry, Prog. Quant. Electr. 16, 251 (1992); C. Albrecht, J. H. Smet, D. Weiss, K. von Klitzing, R. Hennig, M. Langenbuch, M. Suhrke, U. Rössler, V. Umansky, H. Schweizer, Phys. Rev. Lett. 83, 2234 (1999); T. Schlösser, K. Ensslin, J. P. Kotthaus, M. Holland, Europhys. Lett. 33, 683 (1996); C. Albrecht, J. H. Smet, K. von Klitzing, D. Weiss, V. Umansky, and H. Schweizer, Phys. Rev. Lett. 86, 147 (2001); M. C. Geisler, J. H. Smet, V. Umansky, K. von Klitzing, B. Naundorf, R. Ketzmerick, and H. Schweizer, Phys. Rev. Lett. 92, 256801 (2004).

[69] I. Dana and J. Zak, Phys. Rev. B 323612 (1985).

[70] D. Xiao, M. C. Chang and Q. Niu, Rev. Mod. Phys. 82, 1959 (2010).

[71] I. Dana, Y. Avron and J. Zak, J. Phys. C: Solid State Phys. 18, L679 (1985).

[72] D. J. Thouless, M. Kohmoto, M. P. Nightingale, and M. den Nijs, Phys. Rev. Lett. 49, 405 (1982).

[73] M. C. Chang and Q. Niu, Phys. Rev. Lett. 75, 1348 (1995).

[74] B. Sachs, T. O. Wehling, M. I. Katsnelson, A. I. Lichtenstein, Phys. Rev. B 84, 195414 (2011).

[75] A. B. Kuzmenko, I. Crassee, D. van der Marel, P. Blake, K. S. Novoselov, Phys. Rev. B 80, 165406 (2009).

[76] K. Kechedzhi, V. I. Fal'ko, E. McCann, B. L. Altshuler, Phys. Rev. Lett. 98, 176806 (2007).

[77] Y. Lemonik, I. L. Aleiner, V. I. Fal'ko, Phys. Rev. B 85, 245451 (2012).

[78] E. McCann, Phys. Rev. B 74, 161403 (2006). 
[79] M. Mucha-Kruczynski, E. McCann, V. I. Fal'ko, Sol. St. Commun. 149, 1111 (2009).

[80] S. Marchini, S. Günther, and J. Wintterlin, Phys. Rev. B 76, 075429 (2007).

[81] A. T. N’Diaye , J. Coraux, T. N. Plasa, C. Busse and T. Michely, New J. Phys. 10, 043033 (2008).

[82] V. V. Cheianov , V. Falko, O. Syljuåsen , B. L. Altshuler, Sol. St. Commun. 149, 1499 (2009).

[83] O. Madelung (editor), Semiconductors: Group IV Elements and III-V Compounds in the series Data in Science and Technology, Springer-Verlag Berlin 1991.

[84] S. Lebegue, T. Bjorkman, M. Klintenberg, R. M. Nieminen, and O. Eriksson, Phys. Rev. X 3, 031002 (2013).

[85] J. A. Wilson and A. D. Yoffe, Advances in Physics 18, 193 (1969).

[86] S. Furuseth, K. Selte, and A. Kjeksiius, Acta chem. scand. 19, 257. (1965).

[87] J. V. McCanny and R. B. Murray, J. Phys. C: Solid State Phys. 10, 1211 (1977).

[88] V. Zólyomi, N. D. Drummond, and V. Fal'ko, Phys. Rev. B 87, 195403 (2013).

[89] S. A. Semiletov and V. A. Vlasov, Sov. Phys. Crystallogr. 8, 704 (1964).

[90] E. G. Gillan and A. R. Barron, Chem. Mater. 9, 3037 (1997).

[91] C. B. M. Andersson, U. O. Karlsson, M. C. Hakansson, L. O. Olsson, L. Ilver, J. Kanski, P.-O. Nilsson, and P. E. S. Persson, Surf. Sci. 307-309, 885 (1994).

[92] X.-Y. Hou, G.-S. Dong, X.-M. Ding, and X. Wang, Surf. Sci. 183, 123 (1987).

[93] S. Mankefors, P. O. Nilsson, J. Kanski, Surf. Sci. 443, L1049 (1999). 
[94] K. Szamota-Leandersson, M. Gothelid, J. Kanski, L. Ilver, G. Le Lay, and U. O. Karlsson, Phys. Rev. B 74, 205406 (2006).

[95] Y.-J. Yu, Y. Zhao, S. Ryu, L. E. Brus, K. S. Kim, and P. Kim, Nano Letters 9, $3430(2009)$.

[96] Y. Fukuda, S. Ichikawa, M. Shimomura, N.Sanada, and Y. Suzuki, Vacuum 67,37 (2002).

[97] M. J. Lowe, T. D. Veal, A. P. Mowbray, and C. F. McConville, Surf. Sci. 544, 320 (2003).

[98] A. C. Ferrari, Solid State Commun. 143, 47 (2007) .

[99] A. H. Castro Neto et al., Rev. Mod. Phys. 81, 109 (2009).

[100] S. Piscanec, M. Lazzeri, F. Mauri, A. C. Ferrari, and J. Robertson, Phys. Rev. Lett. 93, 185503 (2004).

[101] T. Ando, J. Phys. Soc. Jpn. 75, 124701 (2006).

[102] M. Lazzeri and F. Mauri, Phys. Rev. Lett. 97, 266407 (2006).

[103] A. H. Castro Neto and F. Guinea, Phys. Rev. B 75, 045404 (2007).

[104] M. O. Goerbig et al., Phys. Rev. Lett. 99, 087402 (2007).

[105] T. Ando, J. Phys. Soc. Jpn. 76, 024712 (2007).

[106] D. M. Basko, Phys. Rev. B 76, 081405(R) (2007).

[107] D. M. Basko and I. L. Aleiner, Phys. Rev. B 77, 041409 (2008).

[108] D. M. Basko, S. Piscanec, and A. C. Ferrari, Phys. Rev. B 80, 165413 (2009).

[109] A. C. Ferrari et al., Phys. Rev. Lett. 97, 187401 (2006).

[110] A. Gupta et al., Nano Lett. 6, 2667 (2006).

[111] D. Graf et al., Nano Lett. 7, 238 (2007). 
[112] J. Yan et al., Phys. Rev. Lett. 98, 166802 (2007).

[113] S. Pisana et al., Nat. Mater. 6, 198 (2007).

[114] A. Grüneis et al., Phys. Rev. B 80, 085423 (2009).

[115] C. Faugeras et al., Phys. Rev. B 81, 155436 (2010).

[116] C. Faugeras et al., Phys. Rev. Lett. 103, 186803 (2009).

[117] A. Eckmann et al., Nano Lett. 13, 5242 (2013).

[118] C.-H. Park et al., Nano Lett. 8, 4229 (2008).

[119] K. M. Borysenko et al., Phys. Rev. B 81, 121412(R) (2010).

[120] L. M. Woods and G. D. Mahan, ibid. 61, 10651 (2000).

[121] H. Suzuura and T. Ando, J. Phys. Soc. Jpn. 77, 044703 (2008).

[122] E. McCann and V. I. Fal'ko, Phys. Rev. Lett. 96, 086805 (2006).

[123] K. Ishikawa and T. Ando, J. Phys. Soc. Jpn. 75, 084713 (2006).

[124] T. Ando, T. Nakanishi, and R. Saito, J. Phys. Soc. Jpn. 67, 2857 (1998).

[125] G. Kresse and J. Furthmüller, Phys. Rev. B 54, 11169 (1996).

[126] B. Shan and K. Cho, Phys. Rev. B 73, 081401(R) (2006).

[127] J. Jiang et al., Chem. Phys. Lett. 392, 383 (2004).

[128] V. Perebeinos, J. Tersoff and P. Avouris, Phys. Rev. Lett. 94, 086802 (2005).

[129] G. D. Mahan, Phys. Rev. B 68, 125409 (2003).

[130] M. Lazzeri et al., Phys. Rev. B 73, 155426 (2006).

[131] R. de Gail, M. O. Goerbig, and G. Montambaux, Phys. Rev. B 86, 045407 (2012). 